DOI 10.4171/JEMS/306

Jonathan Brundan · Catharina Stroppel

\title{
Highest weight categories arising from Khovanov's diagram algebra IV: the general linear supergroup
}

Received July 15, 2009 and in revised form October 15, 2010

Abstract. We prove that blocks of the general linear supergroup are Morita equivalent to a limiting version of Khovanov's diagram algebra. We deduce that blocks of the general linear supergroup are Koszul.

\section{Introduction}

This is the culmination of a series of four articles studying various generalisations of Khovanov's diagram algebra from [Kh]. The goal is to relate the limiting version $H_{r}^{\infty}$ of this algebra constructed in [BS1] to blocks of the general linear supergroup $G L(m \mid n)$. More precisely, working always over a fixed algebraically closed field $\mathbb{F}$ of characteristic zero, we show that any block of $G L(m \mid n)$ of atypicality $r$ is Morita equivalent to the algebra $H_{r}^{\infty}$. We refer the reader to the introduction of [BS1] for a detailed account of our approach to the definition of Khovanov's diagram algebra and the construction of its limiting version; see also [St3] which discusses further the connections to link homology.

To formulate our main result in detail, fix $m, n \geq 0$ and let $G$ denote the algebraic supergroup $G L(m \mid n)$ over $\mathbb{F}$. Using scheme-theoretic language, $G$ can be regarded as a functor from the category of commutative superalgebras over $\mathbb{F}$ to the category of groups, mapping a commutative superalgebra $A=A_{\overline{0}} \oplus A_{\overline{1}}$ to the group $G(A)$ of all invertible $(m+n) \times(m+n)$ matrices of the form

$$
g=\left(\begin{array}{l|l}
a & b \\
\hline c & d
\end{array}\right)
$$

where $a$ (resp. $d$ ) is an $m \times m$ (resp. $n \times n)$ matrix with entries in $A_{\overline{0}}$, and $b$ (resp. $c$ ) is an $m \times n$ (resp. $n \times m)$ matrix with entries in $A_{\overline{1}}$.

We are interested here in finite-dimensional representations of $G$, which can be viewed equivalently as integrable supermodules over its Lie superalgebra $\mathfrak{g} \cong \mathfrak{g l}(m \mid n, \mathbb{F})$;

J. Brundan: Department of Mathematics, University of Oregon, Eugene, OR 97403, USA; e-mail: brundan@uoregon.edu

C. Stroppel: Department of Mathematics, University of Bonn, 53115 Bonn, Germany; e-mail: stroppel@math.uni-bonn.de

Mathematics Subject Classification (2010): 17B10, 16S37 
the condition for integrability is the same as for $\mathfrak{g}_{\overline{0}} \cong \mathfrak{g l}(m, \mathbb{F}) \oplus \mathfrak{g l}(n, \mathbb{F})$. For example, we have the natural $G$-module $V$ of column vectors, with standard basis $v_{1}, \ldots, v_{m}, v_{m+1}$, $\ldots, v_{m+n}$ and $\mathbb{Z}_{2}$-grading defined by putting $v_{r}$ in degree $\bar{r}:=\overline{0}$ if $1 \leq r \leq m, \bar{r}:=\overline{1}$ if $m+1 \leq r \leq m+n$. Let $B$ and $T$ be the standard choices of Borel subgroup and maximal torus: for each commutative superalgebra $A$, the groups $B(A)$ and $T(A)$ consist of all matrices $g \in G(A)$ that are upper triangular and diagonal, respectively. Let $\varepsilon_{1}, \ldots, \varepsilon_{m+n}$ be the usual basis for the character group $X(T)$ of $T$, i.e. $\varepsilon_{r}$ picks out the $r$ th diagonal entry of a diagonal matrix. Equip $X(T)$ with a symmetric bilinear form $(\cdot, \cdot)$ such that $\left(\varepsilon_{r}, \varepsilon_{s}\right)=(-1)^{\bar{r}} \delta_{r, s}$, and set

$$
\rho:=\sum_{r=1}^{m}(1-r) \varepsilon_{r}+\sum_{s=1}^{n}(m-s) \varepsilon_{m+s} .
$$

Let

$$
X^{+}(T):=\left\{\begin{array}{l|c}
\lambda \in X(T) \mid \begin{array}{c}
\left(\lambda+\rho, \varepsilon_{1}\right)>\cdots>\left(\lambda+\rho, \varepsilon_{m}\right), \\
\left(\lambda+\rho, \varepsilon_{m+1}\right)<\cdots<\left(\lambda+\rho, \varepsilon_{m+n}\right)
\end{array}
\end{array}\right\}
$$

denote the set of dominant weights.

We allow only even morphisms between $G$-modules, so that the category of all finitedimensional $G$-modules is obviously an abelian category. Any $G$-module $M$ decomposes as $M=M_{+} \oplus M_{-}$, where $M_{+}$(resp. $M_{-}$) is the $G$-submodule of $M$ spanned by the degree $\bar{\lambda}$ (resp. the degree $\bar{\lambda}+\overline{1}$ ) component of the $\lambda$-weight space of $M$ for all $\lambda \in X(T)$; here $\bar{\lambda}:=\left(\lambda, \varepsilon_{m+1}+\cdots+\varepsilon_{m+n}\right)(\bmod 2)$. It follows that the category of all finitedimensional $G$-modules decomposes as $\mathscr{F} \oplus \Pi \mathscr{F}$, where $\mathscr{F}=\mathscr{F}(m \mid n)$ (resp. $\Pi \mathscr{F}=$ $\Pi \mathscr{F}(m \mid n))$ is the full subcategory consisting of all $M$ such that $M=M_{+}$(resp. $M=$ $\left.M_{-}\right)$. Moreover $\mathscr{F}$ and $\Pi \mathscr{F}$ are obviously equivalent. In view of this decomposition, we will focus just on $\mathscr{F}$ from now on. Note further that $\mathscr{F}$ is closed under tensor product, and it contains both the natural module $V$ and its dual $V^{*}$.

By [B1, Theorem 4.47], the category $\mathscr{F}$ is a highest weight category with weight poset $\left(X^{+}(T), \leq\right)$, where $\leq$ is the Bruhat ordering defined combinatorially in the next paragraph. We fix representatives $\left\{\mathcal{L}(\lambda) \mid \lambda \in X^{+}(T)\right\}$ for the isomorphism classes of irreducible modules in $\mathscr{F}$ so that $\mathcal{L}(\lambda)$ is an irreducible object in $\mathscr{F}$ generated by a one-dimensional $B$-submodule of weight $\lambda$. We also denote the standard and projective indecomposable modules in the highest weight category $\mathscr{F}$ by $\left\{\mathcal{V}(\lambda) \mid \lambda \in X^{+}(T)\right\}$ and $\left\{\mathcal{P}(\lambda) \mid \lambda \in X^{+}(T)\right\}$, respectively. So $\mathcal{P}(\lambda) \rightarrow \mathcal{V}(\lambda) \rightarrow \mathcal{L}(\lambda)$. In this setting, the standard module $\mathcal{V}(\lambda)$ is often referred to as a Kac module after [Ka].

Now we turn our attention to the diagram algebra side. Let $\Lambda=\Lambda(m \mid n)$ denote the set of all weights in the diagrammatic sense of [BS1, §2] drawn on a number line with vertices indexed by $\mathbb{Z}$, such that a total of $m$ vertices are labelled $\times$ or $\vee$, a total of $n$ vertices are labelled $\circ$ or $\vee$, and all of the (infinitely many) remaining vertices are labelled $\wedge$. From now on, we identify the set $X^{+}(T)$ introduced above with the set $\Lambda$ via the following weight dictionary. Given $\lambda \in X^{+}(T)$, we define

$$
\begin{aligned}
& I_{\vee}(\lambda):=\left\{\left(\lambda+\rho, \varepsilon_{1}\right), \ldots,\left(\lambda+\rho, \varepsilon_{m}\right)\right\}, \\
& I_{\wedge}(\lambda):=\mathbb{Z} \backslash\left\{\left(\lambda+\rho, \varepsilon_{m+1}\right), \ldots,\left(\lambda+\rho, \varepsilon_{m+n}\right)\right\} .
\end{aligned}
$$


Then we identify $\lambda$ with the element of $\Lambda$ whose $i$ th vertex is labelled

$$
\begin{cases}\circ & \text { if } i \text { does not belong to either } I_{\vee}(\lambda) \text { or } I_{\wedge}(\lambda), \\ \vee & \text { if } i \text { belongs to } I_{\vee}(\lambda) \text { but not to } I_{\wedge}(\lambda), \\ \wedge & \text { if } i \text { belongs to } I_{\wedge}(\lambda) \text { but not to } I_{\vee}(\lambda), \\ \times & \text { if } i \text { belongs to both } I_{\vee}(\lambda) \text { and } I_{\wedge}(\lambda) .\end{cases}
$$

For example, the zero weight (which parametrises the trivial $G$-module) is identified with the diagram

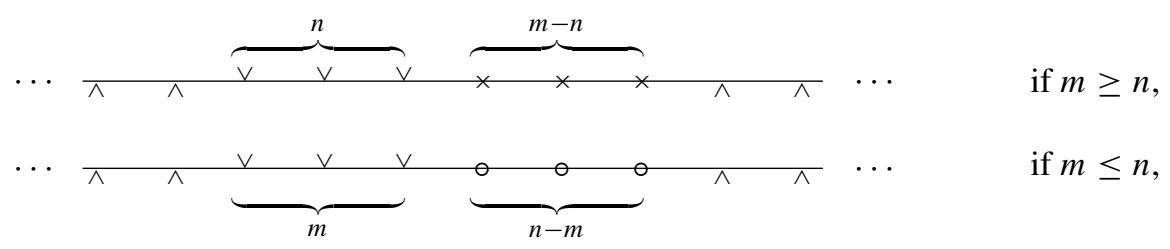

where the leftmost $\vee$ is on vertex $1-m$. In these diagrammatic terms, the Bruhat ordering on $X^{+}(T)$ mentioned earlier is the same as the Bruhat ordering on $\Lambda$ from [BS1, §2], that is, the partial order $\leq$ on diagrams generated by the basic operation of swapping a $\vee$ and an $\wedge$ so that $\vee$ 's move to the right.

Let $\sim$ be the equivalence relation on $\Lambda$ generated by permuting $\vee$ 's and $\wedge$ 's. Following the language of [BS1] again, the $\sim$-equivalence classes of weights from $\Lambda$ are called blocks. The defect $\operatorname{def}(\Gamma)$ of each block $\Gamma \in \Lambda / \sim$ is simply equal to the number of vertices labelled $\vee$ in any weight $\lambda \in \Gamma$; this is the same thing as the usual notion of atypicality in the representation theory of $G L(m \mid n)$ as in e.g. [Se1, (1.1)].

Let $K=K(m \mid n)$ denote the direct sum of the diagram algebras $K_{\Gamma}$ associated to all the blocks $\Gamma \in \Lambda / \sim$ as defined in [BS1, §4]. As a vector space, $K$ has a basis

$$
\{(a \lambda b) \mid \text { for all oriented circle diagrams } a \lambda b \text { with } \lambda \in \Lambda\},
$$

and its multiplication is defined by an explicit combinatorial procedure in terms of such diagrams as in [BS1, §6]; see the discussion at the end of this introduction for some examples illustrating the precise meaning of all this. As explained in [BS1, §5], to each $\lambda \in \Lambda$ there is associated an idempotent $e_{\lambda} \in K$. The left ideal $P(\lambda):=K e_{\lambda}$ is a projective indecomposable module with irreducible head denoted $L(\lambda)$. The modules $\{L(\lambda) \mid \lambda \in \Lambda\}$ are all one-dimensional and give a complete set of irreducible $K$-modules. Finally let $V(\lambda)$ be the standard module corresponding to $\lambda$, which was referred to as a cell module in [BS1, §5].

The main result of the paper is the following.

Theorem 1.1. There is an equivalence of categories $\mathbb{E}$ from $\mathscr{F}(m \mid n)$ to the category of finite-dimensional left $K(m \mid n)$-modules such that $\mathbb{E} \mathcal{L}(\lambda) \cong L(\lambda), \mathbb{E} \mathcal{V}(\lambda) \cong V(\lambda)$ and $\mathbb{E} \mathcal{P}(\lambda) \cong P(\lambda)$ for each $\lambda \in \Lambda(m \mid n)$.

Our proof of Theorem 1.1 involves showing that $K$ is isomorphic to the locally finite endomorphism algebra $\operatorname{End}_{G}^{\mathrm{fin}}(P)^{\mathrm{op}}$ of a canonical minimal projective generator $P \cong$ 
$\bigoplus_{\lambda \in \Lambda} \mathcal{P}(\lambda)$ for $\mathscr{F}$; see Lemmas 5.8-5.9 below. To construct $P$, we first consider the weight

$$
\lambda_{p, q}:=\sum_{r=1}^{m} p \varepsilon_{r}-\sum_{s=1}^{n}(q+m) \varepsilon_{m+s}
$$

for integers $p \leq q$. This is represented diagrammatically by

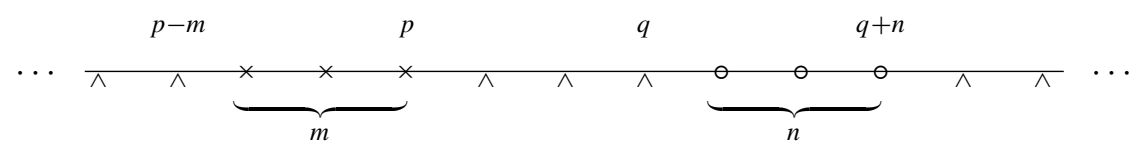

where the rightmost $\times$ is on vertex $p$ and the rightmost $\circ$ is on vertex $q+n$. The $G$-module $\mathcal{V}\left(\lambda_{p, q}\right)$ is projective, hence the "tensor space" $\mathcal{V}\left(\lambda_{p, q}\right) \otimes V^{\otimes d}$ is projective for any $d \geq 0$. Moreover, any $\mathcal{P}(\lambda)$ appears as a summand of $\mathcal{V}\left(\lambda_{p, q}\right) \otimes V^{\otimes d}$ for suitable $p, q$ and $d$. The key step in our approach is to compute the endomorphism algebra of $\mathcal{V}\left(\lambda_{p, q}\right) \otimes V^{\otimes d}$ for $d \geq 0$. For $d \leq \min (m, n)$, we show that it is a certain degenerate cyclotomic Hecke algebra of level two, giving a new "super" version of the level two Schur-Weyl duality from [BK1]. Then we invoke results from [BS3] which show that the basic algebra that is Morita equivalent to this cyclotomic Hecke algebra is a generalised Khovanov algebra; this equivalence relies in particular on the connection between cyclotomic Hecke algebras and Khovanov-Lauda-Rouquier algebras in type $A$ from [BK2]. Finally we let $p, q$ and $d$ vary, taking a suitable direct limit to derive our main result.

We briefly collect here some applications of Theorem 1.1.

\section{Blocks of the same atypicality are equivalent}

The algebras $K_{\Gamma}$ for all $\Gamma \in \Lambda(m \mid n) / \sim$ are the blocks of the algebra $K(m \mid n)$. Hence by Theorem 1.1 they are the basic algebras representing the individual blocks of the category $\mathscr{F}(m \mid n)$. In the diagrammatic setting, it is obvious for $\Gamma \in \Lambda(m \mid n) / \sim$ and $\Gamma^{\prime} \in \Lambda\left(m^{\prime} \mid n^{\prime}\right) / \sim$ (for possibly different $m^{\prime}$ and $n^{\prime}$ ) that the algebras $K_{\Gamma}$ and $K_{\Gamma^{\prime}}$ are isomorphic if and only if $\Gamma$ and $\Gamma^{\prime}$ have the same defect. Thus we recover a result of Serganova from [Se2]: the blocks of $G L(m \mid n)$ for all $m, n$ depend up to equivalence only on the degree of atypicality of the block.

\section{Gradings on blocks and Koszulity}

Each of the algebras $K_{\Gamma}$ carries a canonical positive grading with respect to which it is a (locally unital) Koszul algebra; see [BS2, Corollary 5.13]. So Theorem 1.1 implies that blocks of $G L(m \mid n)$ are Koszul. The appearence of such hidden Koszul gradings in representation theory goes back to the classic paper of Beilinson, Ginzburg and Soergel [BGS] on blocks of category $\mathcal{O}$ for a semisimple Lie algebra. In that work, the grading is of geometric origin, whereas in our situation we establish the Koszulity in a purely algebraic way. 


\section{Rigidity of Kac modules}

Another consequence of Theorem 1.1, combined with [BS2, Corollary 6.7] on the diagram algebra side, is that all the Kac modules $\mathcal{V}(\lambda)$ are rigid, i.e. their radical and socle filtrations coincide. See [BS1, Theorem 5.2] for the explicit combinatorial description of the layers.

\section{Kostant modules and $B G G$ resolution}

In [BS2] we studied in detail the Kostant modules for the generalised Khovanov algebras, i.e. the irreducible modules whose Kazhdan-Lusztig polynomials are multiplicity-free. In particular in [BS2, Lemma 7.2] we classified the highest weights of these modules via a pattern avoidance condition. Combining this with Theorem 1.1, we obtain the following classification of all Kostant modules for $G L(m \mid n)$ : they are the irreducible modules parametrised by the weights in which no two vertices labelled $\vee$ have a vertex labelled $\wedge$ between them. By [BS2, Theorem 7.3], Kostant modules possess a BGG resolution by multiplicity-free direct sums of standard modules. All irreducible polynomial representations of $G L(m \mid n)$ satisfy the combinatorial criterion to be Kostant modules, so this gives another proof of the main result of [CKL].

\section{Endomorphism algebras of PIMs}

For any $\lambda \in \Lambda$, Theorem 1.1 implies that the endomorphism algebra $\operatorname{End}_{G}(\mathcal{P}(\lambda))^{\text {op }}$ of the projective indecomposable module $\mathcal{P}(\lambda)$ is isomorphic to the algebra $e_{\lambda} K e_{\lambda}$. By the definition of multiplication in $K$, this algebra is isomorphic to $\mathbb{F}\left[x_{1}, \ldots, x_{r}\right] /\left(x_{1}^{2}, \ldots, x_{r}^{2}\right)$ where $r$ is the defect (atypicality) of the block containing $\lambda$, answering a question raised recently by several authors; see [BKN, (4.2)] and [Dr, Conjecture 4.3.3]. (It should also be possible to give a proof of the commutativity of these endomorphism algebras using some deformation theory as in [St2, §2.8], invoking the fact that the multiplicities $(\mathcal{P}(\lambda): \mathcal{V}(\mu))$ are at most one by Theorem 2.1 below; see [St1, Theorem 7.1$]$ for a similar situation.)

\section{Super duality}

When combined with the results from [BS3], our results can be used to prove the "Super Duality Conjecture" as formulated in [CWZ]. A direct algebraic proof of this conjecture, and its substantial generalisation from $[\mathrm{CW}]$, has recently been found by Cheng and Lam [CL]. All of these results suggest that some more direct geometric connection between the representation theory of $G L(m \mid n)$ and the category of perverse sheaves on Grassmannians may exist.

To conclude this introduction, we recall in more detail the definition of the algebra $K$ following [BS1, §6]. We assume $m=n=r$ and focus just on the principal block of $G=G L(r \mid r)$, which is the basic example of a block of atypicality $r$. The dominant 
weights in this block are the weights

$$
\lambda_{i_{1}, \ldots, i_{r}}:=\sum_{s=1}^{r}\left(i_{s}+s-1\right)\left(\varepsilon_{s}-\varepsilon_{2 r+1-s}\right) \in X^{+}(T)
$$

parametrised by sequences $i_{1}>\cdots>i_{r}$ of integers. According to the weight dictionary (1.6), the diagram for $\lambda_{i_{1}, \ldots, i_{r}}$ has label $\vee$ at the vertices indexed by $i_{1}, \ldots, i_{r}$, and label $\wedge$ at all remaining vertices. The corresponding block of the algebra $K$ is exactly the algebra denoted $K_{r}^{\infty}$ in the introduction of [BS1]. Theorem 1.1 (or rather the more precise Lemmas 5.8-5.9 below) asserts in this situation that

$$
K_{r}^{\infty} \cong \operatorname{End}_{G}^{\mathrm{fin}}\left(\bigoplus_{i_{1}>\cdots>i_{r}} \mathcal{P}\left(\lambda_{i_{1}, \ldots, i_{r}}\right)\right)^{\text {op }} .
$$

Our explicit basis of $K_{r}^{\infty}$ is given by the oriented circle diagrams from (1.7). These are obtained by taking the diagram of some $\lambda_{i_{1}, \ldots, i_{r}}$, then gluing $r$ cups and infinitely many rays to the bottom and $r$ caps and infinitely many rays to the top of the diagram so that

- every vertex meets exactly one cup or ray below and exactly one cap or ray above the number line;

- each cup and each cap is incident with one vertex labelled $\wedge$ and one vertex labelled $\vee$;

- each ray is incident with a vertex labelled $\wedge$ and extends from there vertically up or down to infinity;

- no crossings of cups, caps and rays are allowed.

Under the isomorphism (1.10), such a diagram represents a homomorphism $\mathcal{P}\left(\lambda_{j_{1}, \ldots, j_{r}}\right)$ $\rightarrow \mathcal{P}\left(\lambda_{k_{1}, \ldots, k_{r}}\right)$ where $j_{1}>\cdots>j_{r}$ (resp. $k_{1}>\cdots>k_{r}$ ) index the leftmost vertices of the cups (resp. caps) in the diagram. For example, here are two oriented circle diagrams corresponding to basis vectors in $K_{2}^{\infty}$ (where $\cdots$ indicates infinitely many pairs of vertical rays labelled $\wedge$ ):
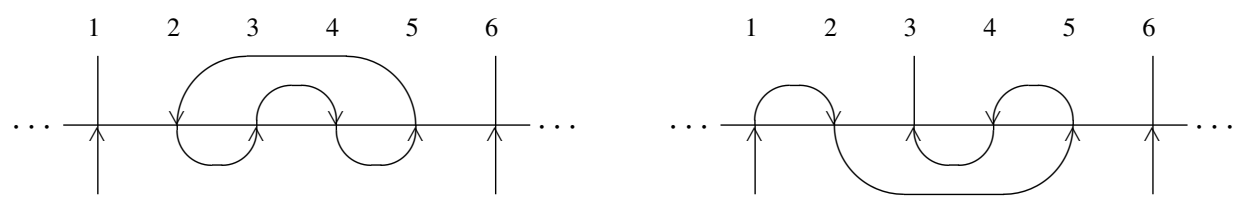

The first diagram here represents a homomorphism $\mathcal{P}\left(\lambda_{4,2}\right) \rightarrow \mathcal{P}\left(\lambda_{3,2}\right)$ and the second one represents a homomorphism $\mathcal{P}\left(\lambda_{3,2}\right) \rightarrow \mathcal{P}\left(\lambda_{4,1}\right)$. Multiplying these two basis vectors together as described in the next paragraph, bearing in mind the "op" in (1.10) which means for once that we are writing maps on the right, one gets the basis vector

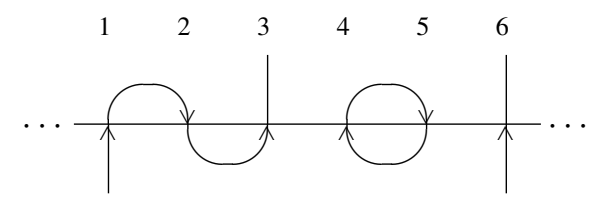

which is some homomorphism $\mathcal{P}\left(\lambda_{4,2}\right) \rightarrow \mathcal{P}\left(\lambda_{4,1}\right)$. 
We now sketch briefly the combinatorial procedure for multiplying basis vectors. Given two basis vectors, their product is necessarily zero unless the caps at the top of the first diagram are in exactly the same positions as the cups at the bottom of the second. Assuming that is the case, we glue the first diagram underneath the second and join matching pairs of rays. Then we perform a sequence of generalised surgery procedures to smooth out all cup-cap pairs in the symmetric middle section of the resulting composite diagram, obtaining zero or more new diagrams in which the middle section only involves vertical line segments. Finally we collapse these middle sections to obtain a sum of basis vectors, which is the desired product. Each generalised surgery procedure in this algorithm either involves two components in the diagram merging into one, or one component splitting into two. The rules for relabelling the new component(s) produced when this operation is performed are summarized as follows:

$$
\begin{array}{rll}
1 \otimes 1 \mapsto 1, & 1 \otimes x \mapsto x, & x \otimes x \mapsto 0, \\
1 \otimes y \mapsto y, & x \otimes y \mapsto 0, & y \otimes y \mapsto 0, \\
1 \mapsto 1 \otimes x+x \otimes 1, & x \mapsto x \otimes x, & y \mapsto x \otimes y,
\end{array}
$$

where 1 represents an anti-clockwise circle, $x$ represents a clockwise circle, and $y$ represents a line. This is a little cryptic; we refer the reader to [BS1] for a fuller account (and explanation of the connection to Khovanov's original construction via a certain TQFT). Let us at least apply this algorithm to the example from the previous paragraph: two surgeries are needed, the first of which involves an anti-clockwise circle and a line merging together $(1 \otimes y \mapsto y)$ and the second of which involves a line splitting into a clockwise circle and a line $(y \mapsto x \otimes y)$ :

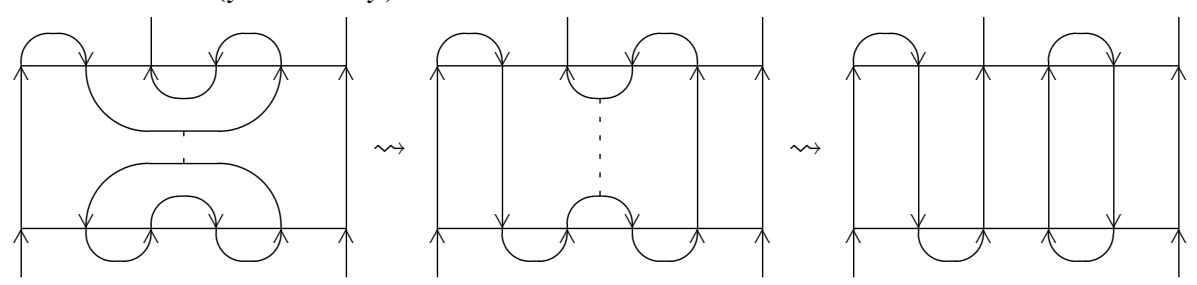

Contracting the middle section of the diagram on the right hand side here gives the final product recorded already at the end of the previous paragraph.

The case $r=1$ in the above discussion (the principal block of $G L(1 \mid 1)$ ) is easy to derive from scratch, but still this is quite instructive. So now the irreducible modules are indexed simply by the weights $\left\{\lambda_{i} \mid i \in \mathbb{Z}\right\}$. It is well known that $\mathcal{P}\left(\lambda_{i}\right)$ has irreducible socle and head isomorphic to $\mathcal{L}\left(\lambda_{i}\right)$, with $\operatorname{rad} \mathcal{P}\left(\lambda_{i}\right) / \operatorname{soc} \mathcal{P}\left(\lambda_{i}\right) \cong \mathcal{L}\left(\lambda_{i-1}\right) \oplus$ $\mathcal{L}\left(\lambda_{i+1}\right)$. Hence in this case the locally finite endomorphism algebra from (1.10) has basis $\left\{e_{i}, c_{i}, a_{i}, b_{i} \mid i \in \mathbb{Z}\right\}$, where $e_{i}$ is the projection onto $\mathcal{P}\left(\lambda_{i}\right), a_{i}: \mathcal{P}\left(\lambda_{i}\right) \rightarrow \mathcal{P}\left(\lambda_{i+1}\right)$ and $b_{i}: \mathcal{P}\left(\lambda_{i+1}\right) \rightarrow \mathcal{P}\left(\lambda_{i}\right)$ are non-zero homomorphisms chosen so that $b_{i} \circ a_{i}=a_{i-1} \circ b_{i-1}$, and $c_{i}:=b_{i} \circ a_{i}$ sends the head of $\mathcal{P}\left(\lambda_{i}\right)$ onto its socle. This corresponds to our diagram basis for $K_{1}^{\infty}$ so that<smiles>[CH]=C1CCCC(C(C)(C)C)CC1</smiles><smiles>[CH]=C1CCCC(C)CC1</smiles><smiles>[CH]=C(C)CCCCCCC</smiles><smiles>CCCCCCC(C)(C)C</smiles> 
where we display only vertices $i, i+1, i+2$ and there are infinitely many pairs of vertical rays labelled $\wedge$ at all other vertices. In fact, $K_{1}^{\infty}$ is simply the path algebra of the infinite quiver

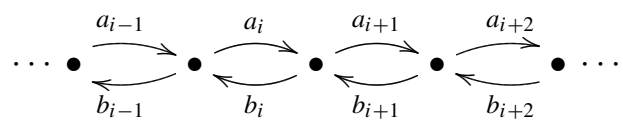

modulo the relations $a_{i} b_{i}=b_{i-1} a_{i-1}$ and $a_{i} a_{i+1}=0=b_{i+1} b_{i}$ for all $i \in \mathbb{Z}$. It is clear from the quiver description that $K_{1}^{\infty}$ is naturally graded by path length; this is actually a Koszul grading. For general $r$ the canonical Koszul grading on $K_{r}^{\infty}$ is defined by declaring that a basis vector is of degree equal to the total number of clockwise cups and caps in the oriented circle diagram.

\section{Combinatorics of Grothendieck groups}

In this preliminary section, we compare the combinatorics underlying the representation theory of $G L(m \mid n)$ with that of the diagram algebra $K(m \mid n)$. Our exposition is largely independent of [B1], indeed, we will reprove the relevant results from there as we go. On the other hand, we do assume that the reader is familiar with the general theory of diagram algebras developed in [BS1, BS2]. Later in the article we will also need to appeal to various results from [BS3].

\section{Representation theory of $K(m \mid n)$}

Fix once and for all integers $m, n \geq 0$. Let $K=K(m \mid n)$ and $\Lambda=\Lambda(m \mid n)$ be as in the introduction. The elements $\left\{e_{\lambda} \mid \lambda \in \Lambda\right\}$ form a system of (in general infinitely many) mutually orthogonal idempotents in $K$ such that

$$
K=\bigoplus_{\lambda, \mu \in \Lambda} e_{\lambda} K e_{\mu}
$$

So the algebra $K$ is locally unital, but it is not unital (except in the trivial case $m=n=0$ ). By a $K$-module we always mean a locally unital module; for a left $K$-module $M$ this means that $M$ decomposes as

$$
M=\bigoplus_{\lambda \in \Lambda} e_{\lambda} M
$$

The irreducible $K$-modules $\{L(\lambda) \mid \lambda \in \Lambda\}$ defined in the introduction are all onedimensional, so $K$ is a basic algebra.

Let $\operatorname{rep}(K)$ denote the category of finite-dimensional left $K$-modules. The Grothendieck group $[\operatorname{rep}(K)]$ of this category is the free $\mathbb{Z}$-module on the basis $\{[L(\lambda)] \mid \lambda \in \Lambda\}$. The standard modules $\{V(\lambda) \mid \lambda \in \Lambda\}$ and the projective indecomposable modules $\{P(\lambda) \mid \lambda \in \Lambda\}$ from [BS1, §5] are finite-dimensional, so it makes sense to consider their classes $[V(\lambda)]$ and $[P(\lambda)]$ in $[\operatorname{rep}(K)]$. Finally, we use the notation $\mu \supset \lambda$ (resp. $\mu \subset \lambda$ ) from [BS1, §2] to indicate that the composite diagram $\mu \bar{\lambda}$ (resp. $\mu \lambda$ ) is oriented in the obvious sense. 
Theorem 2.1. In $[\operatorname{rep}(K)]$ we have

$$
[P(\lambda)]=\sum_{\mu \supset \lambda}[V(\mu)], \quad[V(\lambda)]=\sum_{\mu \subset \lambda}[L(\mu)]
$$

for each $\lambda \in \Lambda$.

Proof. This follows from [BS1, Theorems 5.1 and 5.2].

As $\mu \supset \lambda$ (resp. $\mu \subset \lambda$ ) implies that $\mu \geq \lambda$ (resp. $\mu \leq \lambda$ ) in the Bruhat ordering, we deduce from Theorem 2.1 that the classes $\{[P(\lambda)]\}$ and $\{[V(\lambda)]\}$ are linearly independent in $[\operatorname{rep}(K)]$. However they do not $\operatorname{span}[\operatorname{rep}(K)]$ as the chains in the Bruhat order are infinite.

Remark 2.2. The algebra $K$ possesses a natural $\mathbb{Z}$-grading defined by declaring that each basis vector $(a \lambda b)$ from (1.7) is of degree equal to the number of clockwise cups and caps in the diagram $a \lambda b$. This means that one can consider the graded representation theory of $K$. The various modules $L(\lambda), V(\lambda)$ and $P(\lambda)$ also possess canonical gradings, as is discussed in detail in [BS1, §5].

\section{Special projective functors: the diagram side}

As in [BS3, (2.5)], let us represent a block $\Gamma \in \Lambda / \sim$ by means of its block diagram, that is, the diagram obtained by taking any $\lambda \in \Gamma$ and replacing all the $\Lambda$ 's and $\vee$ 's labelling its vertices by the symbol $\bullet$. Because $m$ and $n$ are fixed, the block $\Gamma$ can be recovered uniquely from its block diagram. Recall also the notion of the defect of a weight $\lambda \in \Lambda$ from [BS1, §2]. In this setting, this simply means the number of vertices labelled $\vee$ in $\lambda$, and the defect of $\lambda$ is the same thing as the defect $\operatorname{def}(\Gamma)$ of the unique block $\Gamma \in \Lambda / \sim$ containing $\lambda$.

Given a block $\Gamma$, we say that $i \in \mathbb{Z}$ is $\Gamma$-admissible if the $i$ th and $(i+1)$ th vertices of the block diagram of $\Gamma$ match the top number line of a unique one of the following pictures, and $\operatorname{def}(\Gamma)$ is as indicated:

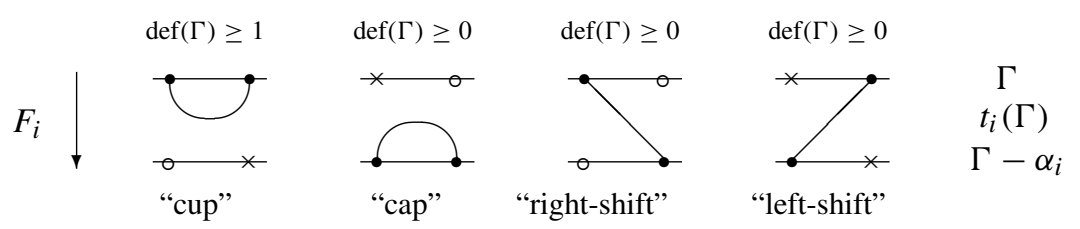

Assuming $i$ is $\Gamma$-admissible, we let $\Gamma-\alpha_{i}$ denote the block obtained from $\Gamma$ by relabelling the $i$ th and $(i+1)$ th vertices of its block diagram according to the bottom number line of the appropriate picture. Also define a $\left(\Gamma-\alpha_{i}\right) \Gamma$-matching $t_{i}(\Gamma)$ in the sense of [BS2, $\S 2]$ so that the strip between the $i$ th and $(i+1)$ th vertices of $t_{i}(\Gamma)$ is as in the picture, and there are only vertical "identity" line segments elsewhere.

For blocks $\Gamma, \Delta \in \Lambda / \sim$ and a $\Gamma \Delta$-matching $t$, recall the geometric bimodule $K_{\Gamma \Delta}^{t}$ from [BS2, §3]. By definition this is a $\left(K_{\Gamma}, K_{\Delta}\right)$-bimodule. We can view it as a $(K, K)$ bimodule by extending the actions of $K_{\Gamma}$ and $K_{\Delta}$ to all of $K$ so that the other blocks act 
as zero. The functor $K_{\Gamma \Delta}^{t} \otimes_{K}$ ? is an endofunctor of $\operatorname{rep}(K)$ called a projective functor. Writing $t^{*}$ for the mirror image of $t$ in a horizontal axis, the functor $K_{\Delta \Gamma}^{t^{*}} \otimes_{K}$ ? gives another projective functor which is biadjoint to $K_{\Gamma \Delta}^{t} \otimes_{K}$ ? by [BS2, Corollary 4.9].

For any $i \in \mathbb{Z}$, introduce the $(K, K)$-bimodules

$$
\widetilde{F}_{i}:=\bigoplus_{\Gamma} K_{\left(\Gamma-\alpha_{i}\right) \Gamma}^{t_{i}(\Gamma)}, \quad \widetilde{E}_{i}:=\bigoplus_{\Gamma} K_{\Gamma\left(\Gamma-\alpha_{i}\right)}^{t_{i}(\Gamma)^{*}},
$$

where the direct sums are over all $\Gamma \in \Lambda / \sim$ such that $i$ is $\Gamma$-admissible. The special projective functors are the endofunctors $F_{i}:=\widetilde{F}_{i} \otimes_{K}$ ? and $E_{i}:=\widetilde{E}_{i} \otimes_{K}$ ? of $\operatorname{rep}(K)$ defined by tensoring with these bimodules. The discussion in the previous paragraph implies that the functors $F_{i}$ and $E_{i}$ are biadjoint, hence they are both exact and map projectives to projectives.

For $\lambda \in \Lambda$, let $I_{\times}(\lambda):=I_{\vee}(\lambda) \cap I_{\wedge}(\lambda)\left(\operatorname{resp} . I_{\circ}(\lambda):=\mathbb{Z} \backslash\left(I_{\vee}(\lambda) \cup I_{\wedge}(\lambda)\right)\right)$ denote the set of integers indexing the vertices labelled $\times$ (resp. $\circ)$ in $\lambda$; cf. (1.6). Introduce the notion of the height of $\lambda$ :

$$
\operatorname{ht}(\lambda):=\sum_{i \in I_{\times}(\lambda)} i-\sum_{i \in I_{\circ}(\lambda)} i
$$

Note all weights belonging to the same block have the same height.

Lemma 2.3. For $\lambda \in \Lambda$ and $i \in \mathbb{Z}$, all composition factors of $F_{i} L(\lambda)\left(\right.$ resp. $\left.E_{i} L(\lambda)\right)$ are of the form $L(\mu)$ with $\mathrm{ht}(\mu)=\mathrm{ht}(\lambda)+1$ (resp. $\mathrm{ht}(\lambda)-1)$.

Proof. This follows by inspecting (2.2).

Lemma 2.4. Let $\lambda \in \Lambda$ and $i \in \mathbb{Z}$. For symbols $x, y \in\{0, \wedge, \vee, \times\}$ we write $\lambda_{x y}$ for the diagram obtained from $\lambda$ by relabelling the ith and $(i+1)$ th vertices by $x$ and $y$, respectively.

(i) If $\lambda=\lambda_{\vee \circ}$ then $F_{i} P(\lambda) \cong P\left(\lambda_{\circ \vee}\right), F_{i} V(\lambda) \cong V\left(\lambda_{\circ \vee}\right), F_{i} L(\lambda) \cong L\left(\lambda_{\circ \vee}\right)$.

(ii) If $\lambda=\lambda_{\wedge \circ}$ then $F_{i} P(\lambda) \cong P\left(\lambda_{\circ \wedge}\right), F_{i} V(\lambda) \cong V\left(\lambda_{\circ \wedge}\right), F_{i} L(\lambda) \cong L\left(\lambda_{\circ \wedge}\right)$.

(iii) If $\lambda=\lambda_{x \vee}$ then $F_{i} P(\lambda) \cong P\left(\lambda_{\vee x}\right), F_{i} V(\lambda) \cong V\left(\lambda_{\vee x}\right), F_{i} L(\lambda) \cong L\left(\lambda_{\vee x}\right)$.

(iv) If $\lambda=\lambda_{\times \wedge}$ then $F_{i} P(\lambda) \cong P\left(\lambda_{\wedge x}\right), F_{i} V(\lambda) \cong V\left(\lambda_{\wedge x}\right), F_{i} L(\lambda) \cong L\left(\lambda_{\wedge x}\right)$.

(v) If $\lambda=\lambda_{\times \circ}$ then:

(a) $F_{i} P(\lambda) \cong P\left(\lambda_{\vee \wedge}\right)$;

(b) there is a short exact sequence

$$
0 \rightarrow V\left(\lambda_{\wedge \vee}\right) \rightarrow F_{i} V(\lambda) \rightarrow V\left(\lambda_{\vee \wedge}\right) \rightarrow 0
$$

(c) $F_{i} L(\lambda)$ has irreducible socle and head both isomorphic to $L\left(\lambda_{\vee \wedge}\right)$, and all other composition factors are of the form $L(\mu)$ for $\mu \in \Lambda$ such that $\mu=\mu_{\mathrm{v}}$, $\mu=\mu_{\wedge \wedge}$ or $\mu=\mu_{\wedge \vee}$.

(vi) If $\lambda=\lambda_{\text {v^ }}$ then $F_{i} P(\lambda) \cong P\left(\lambda_{\circ \times}\right) \oplus P\left(\lambda_{\circ \times}\right), F_{i} V(\lambda) \cong V\left(\lambda_{\circ \times}\right)$ and $F_{i} L(\lambda) \cong$ $L\left(\lambda_{\circ \mathrm{O}}\right)$.

(vii) If $\lambda=\lambda_{\wedge \vee}$ then $F_{i} V(\lambda) \cong V\left(\lambda_{\circ \times}\right)$ and $F_{i} L(\lambda)=\{0\}$.

(viii) If $\lambda=\lambda_{v \vee}$ then $F_{i} V(\lambda)=F_{i} L(\lambda)=\{0\}$. 
(ix) If $\lambda=\lambda_{\wedge \wedge}$ then $F_{i} V(\lambda)=F_{i} L(\lambda)=\{0\}$.

(x) For all other $\lambda$ we have $F_{i} P(\lambda)=F_{i} V(\lambda)=F_{i} L(\lambda)=\{0\}$.

For the dual statement about $E_{i}$, interchange all occurrences of $\circ$ and $\times$.

Proof. Apply [BS2, Theorems 4.2, 4.5 and 4.11], exactly as was done in [BS3, Lemma 3.4].

Remark 2.5. Using Lemma 2.4, one can check that the endomorphisms of $[\operatorname{rep}(K)]$ induced by the functors $F_{i}$ and $E_{i}$ for all $i \in \mathbb{Z}$ satisfy the Serre relations defining the Lie algebra $\mathfrak{s l}_{\infty}$. Indeed, letting $V_{\infty}$ denote the natural $\mathfrak{s l}_{\infty}$-module of column vectors, the category $\operatorname{rep}(K)$ can be interpreted in a precise sense as a categorification of a certain completion of the $\mathfrak{s l}_{\infty}$-module $\bigwedge^{m} V_{\infty} \otimes \bigwedge^{n} V_{\infty}^{*}$; see also [B1] where this point of view is taken on the supergroup side. Using the graded representation theory mentioned in Remark 2.2, i.e. replacing rep $(K)$ with the category of finite-dimensional graded $K$ modules, one gets a categorification of the $q$-analogue of this module over the quantised enveloping algebra $U_{q}\left(\mathfrak{s l}_{\infty}\right)$; the action of $q$ comes from shifting the grading on a module up by one. We are not going to pursue this connection further here, but refer the reader to [BS3, Theorem 3.5] where an analogous "graded categorification theorem" is discussed in detail.

\section{The crystal graph}

Define the crystal graph to be the directed coloured graph with vertex set equal to $\Lambda$ and a directed edge $\mu \stackrel{i}{\rightarrow} \lambda$ of colour $i \in \mathbb{Z}$ if $L(\lambda)$ is a quotient of $F_{i} L(\mu)$. It is clear from Lemma 2.4 that $\mu \stackrel{i}{\rightarrow} \lambda$ if and only if the $i$ th and $(i+1)$ th vertices of $\lambda$ and $\mu$ are labelled according to one of the six cases in the following table, and all other vertices of $\lambda$ and $\mu$ are labelled in the same way:

\begin{tabular}{l|l|l|l|l|l|l}
$\mu$ & $\vee \circ$ & $\wedge \circ$ & $\times \vee$ & $\times \wedge$ & $\times \circ$ & $\vee \wedge$ \\
\hline$\lambda$ & $\circ \vee$ & $\circ \wedge$ & $\vee \times$ & $\wedge \times$ & $\vee \wedge$ & $\circ \times$
\end{tabular}

Comparing this explicit description with [B1, §3-d], it follows that our crystal graph is isomorphic to Kashiwara's crystal graph associated to the $\mathfrak{s l}_{\infty}$-module mentioned in Remark 2.5, which hopefully explains our choice of terminology.

Suppose we are given integers $p \leq q$. Define the following intervals:

$$
\begin{aligned}
& I_{p, q}:=\{p-m+1, p-m+2, \ldots, q+n-1\}, \\
& I_{p, q}^{+}:=\{p-m+1, p-m+2, \ldots, q+n-1, q+n\} .
\end{aligned}
$$

(The reader may find it helpful at this point to note which vertices of the weight $\lambda_{p, q}$ from (1.9) are indexed by the set $I_{p, q}^{+}$.) Then introduce the following subsets of $\Lambda$ :

$$
\begin{aligned}
& \Lambda_{p, q}:=\left\{\lambda \in \Lambda \mid \text { the } i \text { th vertex of } \lambda \text { is labelled } \wedge \text { for all } i \notin I_{p, q}^{+}\right\}, \\
& \Lambda_{p, q}^{\circ}:=\left\{\begin{array}{l|l}
\lambda \in \Lambda_{p, q} & \begin{array}{l}
\text { amongst vertices } j, \ldots, q+n \text { of } \lambda, \text { the number } \\
\text { of } \wedge \text { 's is } \geq \text { the number of } \vee \text { 's, for all } j \in I_{p, q}^{+}
\end{array}
\end{array}\right\} .
\end{aligned}
$$


Note that the weight $\lambda_{p, q}$ from (1.9) belongs to $\Lambda_{p, q}^{\circ}$. It is the unique weight in $\Lambda_{p, q}$ of minimal height.

Lemma 2.6. Given $\lambda \in \Lambda$, choose $p \leq q$ such that $\lambda \in \Lambda_{p, q}^{\circ}$ (which is always possible as there are infinitely many $\wedge$ 's and finitely many $\vee$ 's). Then there are integers $i_{1}, \ldots, i_{d} \in I_{p, q}$, where $d=\operatorname{ht}(\lambda)-\operatorname{ht}\left(\lambda_{p, q}\right)$, such that $\lambda_{p, q} \stackrel{i_{1}}{\rightarrow} \cdots \stackrel{i_{d}}{\rightarrow} \lambda$ is a path in the crystal graph. Moreover

$$
F_{i_{d}} \cdots F_{i_{1}} V\left(\lambda_{p, q}\right) \cong P(\lambda)^{\oplus 2^{r}},
$$

where $r$ is the number of edges in the given path of the form $\vee \wedge \rightarrow \circ \times$

Proof. For the first statement, we proceed by induction on $\operatorname{ht}(\lambda)$. If $\operatorname{ht}(\lambda)=\operatorname{ht}\left(\lambda_{p, q}\right)$, then $\lambda=\lambda_{p, q}$ and the conclusion is trivial. Now assume that $\operatorname{ht}(\lambda)>\operatorname{ht}\left(\lambda_{p, q}\right)$. As $\lambda \in \Lambda_{p, q}^{\circ}$ and $\lambda \neq \lambda_{p, q}$, it is possible to find $i \in I_{p, q}$ such that the $i$ th and $(i+1)$ th vertices of $\lambda$ are labelled $\circ \vee, \circ \wedge, \circ \times, \vee \times, \wedge \times$ or $\vee \wedge$. Inspecting (2.5), there is a unique weight $\mu \in \Lambda_{p, q}^{\circ}$ with $\mu \stackrel{i}{\rightarrow} \lambda$ in the crystal graph. Noting ht $(\mu)=\operatorname{ht}(\lambda)-1$, we are now done by induction. To deduce the second statement, we apply Lemma 2.4 to find easily that $F_{i_{d}} \cdots F_{i_{1}} P\left(\lambda_{p, q}\right) \cong P(\lambda)^{\oplus 2^{r}}$. Finally $P\left(\lambda_{p, q}\right) \cong V\left(\lambda_{p, q}\right)$ as $\lambda_{p, q}$ is of defect zero, by [BS1, Theorem 5.1].

\section{Representation theory of $G L(m \mid n)$}

Now we turn to discussing the representation theory of $G=G L(m \mid n)$. In the introduction, we defined already the abelian category $\mathscr{F}=\mathscr{F}(m \mid n)$ and the irreducible modules $\{\mathcal{L}(\lambda)\}$, the standard modules $\{\mathcal{V}(\lambda)\}$ and the projective indecomposable modules $\{\mathcal{P}(\lambda)\}$, all of which are parametrised by the set $X^{+}(T)$ of dominant weights. We are using an unusual font here (and a few other places later on) to avoid confusion with the analogous $K$ modules $\{L(\lambda)\},\{V(\lambda)\}$ and $\{P(\lambda)\}$. Recall in particular that the $\mathbb{Z}_{2}$-grading on $\mathcal{L}(\lambda)$ is defined so that its $\lambda$-weight space is concentrated in degree $\bar{\lambda}:=\left(\lambda, \varepsilon_{m+1}+\cdots+\varepsilon_{m+n}\right)$ $(\bmod 2)$. Bearing in mind that we consider only even morphisms, the modules

$$
\left\{\mathcal{L}(\lambda) \mid \lambda \in X^{+}(T)\right\} \cup\left\{\Pi \mathcal{L}(\lambda) \mid \lambda \in X^{+}(T)\right\}
$$

give a complete set of pairwise non-isomorphic irreducible $G$-modules, where $\Pi$ denotes the change of parity functor.

The standard module $\mathcal{V}(\lambda)$ is usually called a Kac module in this setting after [Ka], and can be constructed explicitly as follows. Let $P$ be the parabolic subgroup of $G$ such that $P(A)$ consists of all invertible matrices of the form (1.1) with $c=0$, for each commutative superalgebra $A$. Given $\lambda \in X^{+}(T)$, we let $E(\lambda)$ denote the usual finitedimensional irreducible module of highest weight $\lambda$ for the underlying even subgroup $G_{0} \cong G L(m) \times G L(n)$, viewing $E(\lambda)$ as a supermodule with $\mathbb{Z}_{2}$-grading concentrated in degree $\bar{\lambda}$. We can regard $E(\lambda)$ also as a $P$-module by inflating through the obvious homomorphism $P \rightarrow G_{\overline{0}}$. Then

$$
\mathcal{V}(\lambda)=U(\mathfrak{g}) \otimes_{U(\mathfrak{p})} E(\lambda),
$$


where $\mathfrak{g}$ and $\mathfrak{p}$ denote the Lie superalgebras of $G$ and $P$, respectively. This construction makes sense because the induced module on the right hand side of (2.10) is an integrable $\mathfrak{g}$-supermodule, i.e. it lifts in a unique way to a $G$-module.

The module $\mathcal{L}(\lambda)$ is isomorphic to the unique irreducible quotient of $\mathcal{V}(\lambda)$. Also $\mathcal{P}(\lambda)$ is the projective cover of $\mathcal{L}(\lambda)$ in the category $\mathscr{F}$. It has a standard flag, that is, a filtration whose sections are standard modules. The multiplicity $(\mathcal{P}(\lambda): \mathcal{V}(\mu))$ of $\mathcal{V}(\mu)$ as a section of any such standard flag is given by the $B G G$ reciprocity formula

$$
(\mathcal{P}(\lambda): \mathcal{V}(\mu))=[\mathcal{V}(\mu): \mathcal{L}(\lambda)]
$$

as follows from [Zo] or the discussion in [B2, Example 7.5].

\section{Special projective functors: the supergroup side}

Recall the weight dictionary from (1.6) by means of which we identify the set $X^{+}(T)$ with the set $\Lambda=\Lambda(m \mid n)$. Under this identification, the usual notion of the degree of atypicality of a weight $\lambda \in X^{+}(T)$ corresponds to the notion of defect of $\lambda \in \Lambda$. Given $\lambda, \mu \in \Lambda$, the irreducible $G$-modules $\mathcal{L}(\lambda)$ and $\mathcal{L}(\mu)$ have the same central character if and only if $\lambda \sim \mu$ in the diagrammatic sense; this can be deduced from [Se1, Corollary 1.9]. Hence the category $\mathscr{F}$ decomposes as

$$
\mathscr{F}=\bigoplus_{\Gamma \in \Lambda / \sim} \mathscr{F}_{\Gamma},
$$

where $\mathscr{F}_{\Gamma}$ is the full subcategory consisting of the modules all of whose composition factors are of the form $\mathcal{L}(\lambda)$ for $\lambda \in \Gamma$. We let $\mathrm{pr}_{\Gamma}: \mathscr{F} \rightarrow \mathscr{F}$ be the exact functor defined by projection onto $\mathscr{F}_{\Gamma}$ along (2.12).

Recall that $V$ denotes the natural $G$-module and $V^{*}$ is its dual. Following [B1, (4.21)(4.22)], we define the special projective functors $\mathcal{F}_{i}$ and $\mathcal{E}_{i}$ for each $i \in \mathbb{Z}$ to be the following endofunctors of $\mathscr{F}$ :

$$
\mathcal{F}_{i}:=\bigoplus_{\Gamma} \operatorname{pr}_{\Gamma-\alpha_{i}} \circ(? \otimes V) \circ \operatorname{pr}_{\Gamma}, \quad \mathcal{E}_{i}:=\bigoplus_{\Gamma} \operatorname{pr}_{\Gamma} \circ\left(? \otimes V^{*}\right) \circ \operatorname{pr}_{\Gamma-\alpha_{i}},
$$

where the direct sums are over all $\Gamma \in \Lambda / \sim$ such that $i$ is $\Gamma$-admissible (as in (2.3)). The functors $? \otimes V$ and $? \otimes V^{*}$ are biadjoint, hence so are $\mathcal{F}_{i}$ and $\mathcal{E}_{i}$. In particular, all these functors are exact and send projectives to projectives. For later use, let us fix once and for all a choice of an adjunction making $\left(\mathcal{F}_{i}, \mathcal{E}_{i}\right)$ into an adjoint pair for each $i \in \mathbb{Z}$.

Lemma 2.7. The following hold for any $\lambda \in X^{+}(T)$ :

(i) $\mathcal{V}(\lambda) \otimes V$ has a filtration with sections $\mathcal{V}\left(\lambda+\varepsilon_{r}\right)$ for all $r=1, \ldots, m+n$ such that $\lambda+\varepsilon_{r} \in X^{+}(T)$, arranged in order from bottom to top.

(ii) $\mathcal{V}(\lambda) \otimes V^{*}$ has a filtration with sections $\mathcal{V}\left(\lambda-\varepsilon_{r}\right)$ for all $r=1, \ldots, m+n$ such that $\lambda-\varepsilon_{r} \in X^{+}(T)$, arranged in order from top to bottom.

Proof. This follows from the definition (2.10) and the tensor identity. 
Corollary 2.8. The following hold for any $\lambda \in X^{+}(T)$ and $i \in \mathbb{Z}$ :

(i) $\mathcal{F}_{i} \mathcal{V}(\lambda)$ has a filtration with sections $\mathcal{V}\left(\lambda+\varepsilon_{r}\right)$ for all $r=1, \ldots, m+n$ such that $\lambda+\varepsilon_{r} \in X^{+}(T)$ and $\left(\lambda+\rho, \varepsilon_{r}\right)=i+\left(1-(-1)^{\bar{r}}\right) / 2$, arranged in order from bottom to top.

(ii) $\mathcal{E}_{i} \mathcal{V}(\lambda)$ has a filtration with sections $\mathcal{V}\left(\lambda-\varepsilon_{r}\right)$ for all $r=1, \ldots, m+n$ such that $\lambda-\varepsilon_{r} \in X^{+}(T)$ and $\left(\lambda+\rho, \varepsilon_{r}\right)=i+\left(1+(-1)^{\bar{r}}\right) / 2$, arranged in order from top to bottom.

Proof. For (i), apply $\operatorname{pr}_{\Gamma-\alpha_{i}}$ to the statement of Lemma 2.7(i), where $\Gamma$ is the block containing $\lambda$ (and do a little work to translate the combinatorics). The proof of (ii) is similar.

Corollary 2.9. We have ? $\otimes V=\bigoplus_{i \in \mathbb{Z}} \mathcal{F}_{i}$ and $? \otimes V^{*}=\bigoplus_{i \in \mathbb{Z}} \mathcal{E}_{i}$.

The next lemma gives an alternative definition of the functors $\mathcal{F}_{i}$ and $\mathcal{E}_{i}$ which will be needed in the next section; cf. [CW, Proposition 5.2]. Let

$$
\Omega:=\sum_{r, s=1}^{m+n}(-1)^{\bar{s}} e_{r, s} \otimes e_{s, r} \in \mathfrak{g} \otimes \mathfrak{g},
$$

where $e_{r, s}$ denotes the $r s$-matrix unit. This corresponds to the supertrace form on $\mathfrak{g}$, so left multiplication by $\Omega$ (interpreted with the usual superalgebra sign conventions) defines a $G$-module endomorphism of $M \otimes N$ for any $G$-modules $M$ and $N$.

Lemma 2.10. For any $G$-module $M, \mathcal{F}_{i} M$ (resp. $\left.\mathcal{E}_{i} M\right)$ is the generalised $i$-eigenspace (resp. the generalised $-(m-n+i)$-eigenspace) of the operator $\Omega$ acting on $M \otimes V$ (resp. $\left.M \otimes V^{*}\right)$.

Proof. We just consider $\mathcal{F}_{i}$. Let $c:=\sum_{r, s=1}^{m+n}(-1)^{\bar{s}} e_{r, s} e_{s, r} \in U(\mathfrak{g})$ be the Casimir element. It acts on $\mathcal{V}(\lambda)$ by multiplication by the scalar

$$
c_{\lambda}:=(\lambda+2 \rho+(m-n-1) \delta, \lambda)
$$

where $\delta=\varepsilon_{1}+\cdots+\varepsilon_{m}-\varepsilon_{m+1}-\cdots-\varepsilon_{m+n}$. Also, $\Omega=(\Delta(c)-c \otimes 1-1 \otimes c) / 2$ where $\Delta$ is the comultiplication of $U(\mathfrak{g})$. Now to prove the lemma, it suffices to verify it for the special case $M=\mathcal{V}(\lambda)$. Using the observations just made, we see that multiplication by $\Omega$ preserves the filtration from Lemma 2.7(i), and the induced action of $\Omega$ on the section $\mathcal{V}\left(\lambda+\varepsilon_{r}\right)$ is by multiplication by the scalar

$$
\left(c_{\lambda+\varepsilon_{r}}-c_{\lambda}-m+n\right) / 2=\left(\lambda+\rho, \varepsilon_{r}\right)+\left(1-(-1)^{\bar{r}}\right) / 2 .
$$

The result follows on comparing with Corollary 2.8(i).

The next two lemmas are the key to understanding the representation theory of $G L(m \mid n)$ from a combinatorial point of view.

Lemma 2.11. Let $i \in \mathbb{Z}$ and $\lambda \in \Lambda$ be a weight such that the $i$ th and $(i+1)$ th vertices of $\lambda$ are labelled $\wedge$ and $\vee$, respectively. Let $\mu$ be the weight obtained from $\lambda$ by interchanging the labels on these two vertices. Then $\mathcal{L}(\mu)$ is a composition factor of $\mathcal{V}(\lambda)$. 
Proof. This is a reformulation of [Se1, Theorem 5.5]. It can be proved directly by an explicit calculation with certain lowering operators in $U(\mathfrak{g})$ as in [BS3, Lemma 4.8].

Lemma 2.12. Exactly the same statement as Lemma 2.4 holds in the category $\mathscr{F}$, with $L(\lambda), V(\lambda), P(\lambda), F_{i}$ and $E_{i}$ replaced by $\mathcal{L}(\lambda), \mathcal{V}(\lambda), \mathcal{P}(\lambda), \mathcal{F}_{i}$ and $\mathcal{E}_{i}$, respectively.

Proof. The statements involving $\mathcal{V}(\lambda)$ follow from Corollary 2.8. The remaining parts then follow by mimicking the arguments used to prove [BS3, Lemma 4.9], with Lemma 2.11 applied in place of [BS3, Lemma 4.8].

Corollary 2.13. Given $\lambda \in \Lambda$, pick $p, q, d, i_{1}, \ldots, i_{d}$ and $r$ as in Lemma 2.6. Then $\mathcal{F}_{i_{d}} \cdots \mathcal{F}_{i_{1}} \mathcal{V}\left(\lambda_{p, q}\right) \cong \mathcal{P}(\lambda)^{\oplus 2^{r}}$.

Proof. We note as $\lambda_{p, q}$ is of defect zero that it is the only weight in its block. Using also (2.11), this implies that $\mathcal{P}\left(\lambda_{p, q}\right)=\mathcal{V}\left(\lambda_{p, q}\right)$. Given this, the corollary follows from Lemma 2.12 in exactly the same way that Lemma 2.6 was deduced from Lemma 2.4.

\section{Identification of Grothendieck groups}

Consider the Grothendieck group [ $\mathscr{F}$ of $\mathscr{F}$. It is the free $\mathbb{Z}$-module on the basis $\{[\mathcal{L}(\lambda)] \mid$ $\lambda \in \Lambda$. The exact functors $\mathcal{F}_{i}$ and $\mathcal{E}_{i}$ (resp. $F_{i}$ and $E_{i}$ ) induce endomorphisms of the Grothendieck group $[\mathscr{F}$ (resp. $[\operatorname{rep}(K)])$, which we denote by the same notation. The last part of the following theorem recovers the main result of [B1].

Theorem 2.14. Define a $\mathbb{Z}$-module isomorphism $\iota:[\mathscr{F}] \stackrel{\sim}{\rightarrow}[\operatorname{rep}(K)]$ by declaring that $\iota([\mathcal{L}(\lambda)])=[L(\lambda)]$ for each $\lambda \in \Lambda$.

(i) We have $\iota([\mathcal{V}(\lambda)])=[V(\lambda)]$ and $\iota([\mathcal{P}(\lambda)])=[P(\lambda)]$ for each $\lambda \in \Lambda$.

(ii) For each $i \in \mathbb{Z}$, we have $F_{i} \circ \iota=\iota \circ \mathcal{F}_{i}$ and $E_{i} \circ \iota=\iota \circ \mathcal{E}_{i}$ as linear maps from [F] to $[\operatorname{rep}(K)]$.

(iii) In $[\mathscr{F}]$ we have

$$
[\mathcal{P}(\lambda)]=\sum_{\mu \supset \lambda}[\mathcal{V}(\mu)], \quad[\mathcal{V}(\lambda)]=\sum_{\mu \subset \lambda}[\mathcal{L}(\mu)]
$$

for each $\lambda \in \Lambda$.

Proof. Given $\lambda \in \Lambda$, let $p, q, d, r$ and $i_{1}, \ldots, i_{d}$ be as in Lemma 2.6. By Lemma 2.6 and Theorem 2.1, we know already that

$$
[P(\lambda)]=\frac{1}{2^{r}} \cdot F_{i_{d}} \cdots F_{i_{1}}\left[V\left(\lambda_{p, q}\right)\right]=\sum_{\mu \supset \lambda}[V(\mu)],
$$

all equalities written in $[\operatorname{rep}(K)]$. In view of Lemma 2.12, the action of $F_{i}$ on the classes of standard modules in $[\operatorname{rep}(K)]$ is described by exactly the same matrix as the action of $\mathcal{F}_{i}$ on the classes of standard modules in [F्F]. So we deduce from the second equality in (2.15) that

$$
\frac{1}{2^{r}} \cdot \mathcal{F}_{i_{d}} \cdots \mathcal{F}_{i_{1}}\left[\mathcal{V}\left(\lambda_{p, q}\right)\right]=\sum_{\mu \supset \lambda}[\mathcal{V}(\mu)]
$$


equality in $[\mathscr{F}$. By Corollary 2.13 this also equals $[\mathcal{P}(\lambda)]$, proving the first formula in (iii). The second formula in (iii) follows from the first and (2.11).

Then (i) is immediate from the definition of $\iota$ and the coincidence of the formulae in (iii) and Theorem 2.1.

Finally to deduce (ii), we have already noted that $\iota\left(F_{i}[V(\lambda)]\right)=\mathcal{F}_{i}[\mathcal{V}(\lambda)]$ for every $\lambda$. It follows easily from this that $\iota\left(F_{i}[P(\lambda)]\right)=\mathcal{F}_{i}[\mathcal{P}(\lambda)]$ for every $\lambda$. Using also the adjointness of $F_{i}$ and $E_{i}$ (resp. $\mathcal{F}_{i}$ and $\mathcal{E}_{i}$ ) we deduce that

$$
\begin{aligned}
& {\left[E_{i} L(\mu): L(\lambda)\right]=\operatorname{dim} \operatorname{Hom}_{K}\left(P(\lambda), E_{i} L(\mu)\right)} \\
& =\operatorname{dim} \operatorname{Hom}_{K}\left(F_{i} P(\lambda), L(\mu)\right)=\operatorname{dim} \operatorname{Hom}_{G}\left(\mathcal{F}_{i} \mathcal{P}(\lambda), \mathcal{L}(\mu)\right) \\
& =\operatorname{dim} \operatorname{Hom}_{G}\left(\mathcal{P}(\lambda), \mathcal{E}_{i} \mathcal{L}(\mu)\right)=\left[\mathcal{E}_{i} \mathcal{L}(\mu): \mathcal{L}(\lambda)\right]
\end{aligned}
$$

for every $\lambda, \mu \in \Lambda$. This is enough to show that $\iota\left(E_{i}[L(\mu)]\right)=\mathcal{E}_{i}[\mathcal{L}(\mu)]$ for every $\mu$, which implies (ii) for $E_{i}$ and $\mathcal{E}_{i}$. The argument for $F_{i}$ and $\mathcal{F}_{i}$ is similar.

\section{Highest weight structure and duality}

At this point, we can also deduce the following result, which recovers [B1, Theorem 4.47].

Theorem 2.15. The category $\mathscr{F}$ is a highest weight category in the sense of [CPS] with weight poset $(\Lambda, \leq)$. The modules $\{\mathcal{L}(\lambda)\},\{\mathcal{V}(\lambda)\}$ and $\{\mathcal{P}(\lambda)\}$ give its irreducible, standard and projective indecomposable modules, respectively.

Proof. We already noted just before (2.11) that $\mathcal{P}(\lambda)$ has a standard flag with $\mathcal{V}(\lambda)$ at the top. Moreover by Theorem 2.14(iii) the other sections of this flag are all of the form $\mathcal{V}(\mu)$ with $\mu>\lambda$ in the Bruhat order. The theorem follows from this, (2.11) and the definition of highest weight category.

The costandard modules in the highest weight category $\mathscr{F}$ can be constructed explicitly as the duals $\mathcal{V}(\lambda)^{\circledast}$ of the standard modules with respect to a natural duality $\circledast$. This duality maps a $G$-module $M$ to the linear dual $M^{*}$ with the action of $G$ defined using the supertranspose anti-automorphism $g \mapsto g^{s t}$, where

$$
g^{s t}=\left(\begin{array}{c|c}
a^{t} & -c^{t} \\
\hline b^{t} & d^{t}
\end{array}\right)
$$

for $g$ of the form (1.1). Note $\circledast$ fixes irreducible modules, i.e. $\mathcal{L}(\lambda) \circledast \mathcal{L}(\lambda)$ for each $\lambda \in \Lambda$.

\section{Cyclotomic Hecke algebras and level two Schur-Weyl duality}

Fix integers $p \leq q$ and let $\lambda_{p, q}$ be the weight of defect zero from (1.8). The standard module $\mathcal{V}\left(\lambda_{p, q}\right)$ is projective. As the functor ? $\otimes V$ sends projectives to projectives, the $G$-module $\mathcal{V}\left(\lambda_{p, q}\right) \otimes V^{\otimes d}$ is again projective for any $d \geq 0$. We want to describe its endomorphism algebra. 
Action of the degenerate affine Hecke algebra

We begin by constructing an explicit basis for $\mathcal{V}\left(\lambda_{p, q}\right) \otimes V^{\otimes d}$. Recalling (2.10), we have

$$
\mathcal{V}\left(\lambda_{p, q}\right)=U(\mathfrak{g}) \otimes_{U(\mathfrak{p})} E\left(\lambda_{p, q}\right) .
$$

Let $\operatorname{det}_{m}\left(\right.$ resp. $\left.\operatorname{det}_{n}\right)$ denote the one-dimensional $G_{\overline{0}}$-module defined by taking the determinant of $G L(m)$ (resp. $G L(n)$ ), with $\mathbb{Z}_{2}$-grading concentrated in degree $\overline{0}$. Then the module $E\left(\lambda_{p, q}\right)$ in (3.1) is the inflation to $P$ of the module $\Pi^{n(q+m)}\left(\operatorname{det}_{m}^{p} \otimes \operatorname{det}_{n}^{-(q+m)}\right)$, so it is also one-dimensional. Hence, fixing a non-zero highest weight vector $v_{p, q} \in \mathcal{V}\left(\lambda_{p, q}\right)$, the induced module $\mathcal{V}\left(\lambda_{p, q}\right)$ is of dimension $2^{m n}$ with basis

$$
\left\{\prod_{r=m+1}^{m+n} \prod_{s=1}^{m} e_{r, s}^{\tau_{r, s}} \cdot v_{p, q} \mid 0 \leq \tau_{r, s} \leq 1\right\}
$$

where the products are taken in any fixed order (changing the order only changes the vectors by \pm 1 ). Recall also that $v_{1}, \ldots, v_{m+n}$ is the standard basis for the natural module $V$, from which we get the obvious monomial basis

$$
\left\{v_{i_{1}} \otimes \cdots \otimes v_{i_{d}} \mid 1 \leq i_{1}, \ldots, i_{d} \leq m+n\right\}
$$

for $V^{\otimes d}$. Tensoring (3.2) and (3.3), we get the desired basis for $\mathcal{V}\left(\lambda_{p, q}\right) \otimes V^{\otimes d}$.

Now let $H_{d}$ be the degenerate affine Hecke algebra from [D]. This is the associative algebra equal as a vector space to $\mathbb{F}\left[x_{1}, \ldots, x_{d}\right] \otimes \mathbb{F} S_{d}$, the tensor product of a polynomial algebra and the group algebra of the symmetric group $S_{d}$. Multiplication is defined so that $\mathbb{F}\left[x_{1}, \ldots, x_{d}\right] \equiv \mathbb{F}\left[x_{1}, \ldots, x_{d}\right] \otimes 1$ and $\mathbb{F} S_{d} \equiv 1 \otimes \mathbb{F} S_{d}$ are subalgebras of $H_{d}$, and also

$$
s_{r} x_{s}=x_{s} s_{r} \quad \text { if } s \neq r, r+1, \quad s_{r} x_{r+1}=x_{r} s_{r}+1,
$$

where $s_{r}$ denotes the $r$ th basic transposition $(r r+1)$.

By [CW, Proposition 5.1], there is a right action of $H_{d}$ on $\mathcal{V}\left(\lambda_{p, q}\right) \otimes V^{\otimes d}$ by $G$-module endomorphisms. The transposition $s_{r}$ acts as the "super" flip

$\left(v \otimes v_{i_{1}} \otimes \cdots \otimes v_{i_{r}} \otimes v_{i_{r+1}} \otimes \cdots \otimes v_{i_{d}}\right) s_{r}=(-1)^{\bar{i}_{r} \bar{i}_{r+1}} v \otimes v_{i_{1}} \otimes \cdots \otimes v_{i_{r+1}} \otimes v_{i_{r}} \otimes \cdots \otimes v_{i_{d}}$.

This is the same as the endomorphism defined by left multiplication by the element $\Omega$ from (2.14) so that the first and second tensors in $\Omega$ hit the $(r+1)$ th and $(r+2)$ th tensor positions in $\mathcal{V}\left(\lambda_{p, q}\right) \otimes V^{\otimes d}$, respectively. The polynomial generator $x_{s}$ acts by left multiplication by $\Omega$ so that the first tensor in $\Omega$ is spread across tensor positions $1, \ldots, s$ using the comultiplication of $U(\mathfrak{g})$ and the second tensor in $\Omega$ hits the $(s+1)$ th tensor position in $\mathcal{V}\left(\lambda_{p, q}\right) \otimes V^{\otimes d}$. The following lemma gives an explicit formula for the action of $x_{s}$ in a special case.

Lemma 3.1. For $1 \leq i_{1}, \ldots, i_{d} \leq m+n$ and $1 \leq s \leq d$, we have

$$
\begin{aligned}
\left(v_{p, q} \otimes v_{i_{1}} \otimes\right. & \left.\cdots \otimes v_{i_{d}}\right) x_{s}=p v_{p, q} \otimes v_{i_{1}} \otimes \cdots \otimes v_{i_{d}} \\
& +\sum_{r=1}^{s-1}(-1)^{\bar{i}_{r} \bar{i}_{s}+\sum_{r<t<s}\left(\bar{i}_{r}+\bar{i}_{s}\right) \bar{i}_{t}} v_{p, q} \otimes v_{i_{1}} \otimes \cdots \otimes v_{i_{s}} \otimes \cdots \otimes v_{i_{r}} \otimes \cdots \otimes v_{i_{d}}
\end{aligned}
$$




$$
\begin{aligned}
& \text { if } 1 \leq i_{s} \leq m \text {, and } \\
& \begin{aligned}
&\left(v_{p, q} \otimes v_{i_{1}} \otimes \cdots \otimes v_{i_{d}}\right) x_{s}=(q+m) v_{p, q} \otimes v_{i_{1}} \otimes \cdots \otimes v_{i_{d}} \\
& \quad+\sum_{r=1}^{s-1}(-1)^{\bar{i}_{r} \bar{i}_{s}+\sum_{r<t<s}\left(\bar{i}_{r}+\bar{i}_{s}\right) \bar{i}_{t}} v_{p, q} \otimes v_{i_{1}} \otimes \cdots \otimes v_{i_{s}} \otimes \cdots \otimes v_{i_{r}} \otimes \cdots \otimes v_{i_{d}} \\
& \quad+\sum_{j=1}^{m}(-1)^{n(q+m)+\bar{i}_{1}+\cdots+\bar{i}_{s-1}}\left(e_{i_{s}, j} \cdot v_{p, q}\right) \otimes v_{i_{1}} \otimes \cdots \otimes v_{j} \otimes \cdots \otimes v_{i_{d}}
\end{aligned}
\end{aligned}
$$

if $m+1 \leq i_{s} \leq m+n$. (In the first two summations we have interchanged $v_{i_{r}}$ and $v_{i_{s}}$, while in the last one we have replaced $v_{i_{s}}$ by $v_{j}$.)

Proof. Note for any $1 \leq i, j \leq m+n$ that

$$
e_{i, j} \cdot v_{p, q}= \begin{cases}p v_{p, q} & \text { if } 1 \leq i=j \leq m \\ -(q+m) v_{p, q} & \text { if } m+1 \leq i=j \leq m+n \\ e_{i, j} \cdot v_{p, q} & \text { if } m+1 \leq i \leq m+n \text { and } 1 \leq j \leq m, \\ 0 & \text { otherwise. }\end{cases}
$$

Using this, the lemma is a routine calculation (taking care with superalgebra signs).

Corollary 3.2. The element $\left(x_{1}-p\right)\left(x_{1}-q\right) \in H_{d}$ acts as zero on $\mathcal{V}\left(\lambda_{p, q}\right) \otimes V^{\otimes d}$.

Proof. It suffices to check this in the special case $d=1$. In that case, Lemma 3.1 shows that

$$
\left(v_{p, q} \otimes v_{i}\right) x_{1}= \begin{cases}p v_{p, q} \otimes v_{i} & \text { if } 1 \leq i \leq m, \\ q v_{p, q} \otimes v_{i} & \\ +\sum_{j=1}^{m}(-1)^{n(q+m)} e_{i, j}\left(v_{p, q} \otimes v_{j}\right) & \text { if } m+1 \leq i \leq m+n .\end{cases}
$$

It follows easily that $\left(x_{1}-p\right)\left(x_{1}-q\right)$ acts as zero on the vector $v_{p, q} \otimes v_{i}$ for every $1 \leq i \leq m+n$. These vectors generate $\mathcal{V}\left(\lambda_{p, q}\right) \otimes V$ as a $G$-module so we deduce that $\left(x_{1}-p\right)\left(x_{1}-q\right)$ acts as zero on the whole module.

Corollary 3.3. If $d \leq \min (m, n)$ then the endomorphisms of $\mathcal{V}\left(\lambda_{p, q}\right) \otimes V^{\otimes d}$ defined by right multiplication by $\left\{x_{1}^{\sigma_{1}} \cdots x_{d}^{\sigma_{d}} w \mid 0 \leq \sigma_{1}, \ldots, \sigma_{d} \leq 1, w \in S_{d}\right\}$ are linearly independent.

Proof. Any vector $v \in \mathcal{V}\left(\lambda_{p, q}\right) \otimes V^{\otimes d}$ can be written as $v=\sum_{i \in I} b_{i} \otimes c_{i}$ where $\left\{b_{i} \mid i \in I\right\}$ is the basis from (3.2) and the $c_{i}$ 's are unique vectors in $V^{\otimes d}$. We refer to $c_{i}$ as the $b_{i}$-component of $v$. Exploiting the assumption on $d$, we can pick distinct integers $m+1 \leq i_{1}, \ldots, i_{d} \leq m+n$ and $1 \leq j_{1}, \ldots, j_{d} \leq m$. Take $0 \leq \sigma_{1}, \ldots, \sigma_{d} \leq 1$ and consider the vector

$$
\left(v_{p, q} \otimes v_{i_{1}} \otimes \cdots \otimes v_{i_{d}}\right) x_{1}^{\sigma_{1}} \cdots x_{d}^{\sigma_{d}} .
$$

For $0 \leq \tau_{1}, \ldots, \tau_{d} \leq 1$, Lemma 3.1 implies that the $e_{i_{1}, j_{1}}^{\tau_{1}} \cdots e_{i_{d}, j_{d}}^{\tau_{d}} \cdot v_{p, q}$-component of $\left(v_{p, q} \otimes v_{i_{1}} \otimes \cdots \otimes v_{i_{d}}\right) x_{1}^{\sigma_{1}} \cdots x_{d}^{\sigma_{d}}$ is zero either if $\tau_{1}+\cdots+\tau_{d}>\sigma_{1}+\cdots+\sigma_{d}$, or if $\tau_{1}+\cdots+\tau_{d}=\sigma_{1}+\cdots+\sigma_{d}$ but $\tau_{r} \neq \sigma_{r}$ for some $r$. Moreover, if $\tau_{r}=\sigma_{r}$ for all $r$, 
then the $e_{i_{1}, j_{1}}^{\tau_{1}} \cdots e_{i_{d}, j_{d}}^{\tau_{d}} \cdot v_{p, q}$-component of $\left(v_{p, q} \otimes v_{i_{1}} \otimes \cdots \otimes v_{i_{d}}\right) x_{1}^{\sigma_{1}} \cdots x_{d}^{\sigma_{d}}$ is equal to $\pm v_{k_{1}} \otimes \cdots \otimes v_{k_{d}}$ where $k_{r}=i_{r}$ if $\sigma_{r}=0$ and $k_{r}=j_{r}$ if $\sigma_{r}=1$. This is enough to show that the vectors $\left(v_{p, q} \otimes v_{i_{1}} \otimes \cdots \otimes v_{i_{d}}\right) x_{1}^{\sigma_{1}} \cdots x_{d}^{\sigma_{d}} w$ for all $0 \leq \sigma_{1}, \ldots, \sigma_{d} \leq 1$ and $w \in S_{d}$ are linearly independent, and the corollary follows.

In view of Corollary 3.2, the right action of $H_{d}$ on $\mathcal{V}\left(\lambda_{p, q}\right) \otimes V^{\otimes d}$ induces an action of the quotient algebra

$$
H_{d}^{p, q}:=H_{d} /\left\langle\left(x_{1}-p\right)\left(x_{1}-q\right)\right\rangle .
$$

This algebra is a particular example of a degenerate cyclotomic Hecke algebra of level two. It is well known (e.g. see [BK1, Lemma 3.5]) that $\operatorname{dim} H_{d}^{p, q}=2^{d} d$ !.

Corollary 3.4. If $d \leq \min (m, n)$ the action of $H_{d}^{p, q}$ on $\mathcal{V}\left(\lambda_{p, q}\right) \otimes V^{\otimes d}$ is faithful.

Proof. This follows on comparing the dimension of $H_{d}^{p, q}$ with the number of linearly independent endomorphisms constructed in Corollary 3.3.

Since the action of $H_{d}^{p, q}$ on $\mathcal{V}\left(\lambda_{p, q}\right) \otimes V^{\otimes d}$ is by $G$-module endomorphisms, it induces an algebra homomorphism

$$
\Phi: H_{d}^{p, q} \rightarrow \operatorname{End}_{G}\left(\mathcal{V}\left(\lambda_{p, q}\right) \otimes V^{\otimes d}\right)^{\mathrm{op}} .
$$

The main goal in the remainder of the section is to show that this homomorphism is surjective.

Weight idempotents and the space $T_{d}^{p, q}$

For a tuple $i=\left(i_{1}, \ldots, i_{d}\right) \in \mathbb{Z}^{d}$, there is an idempotent $e(i) \in H_{d}^{p, q}$ uniquely determined by the property that multiplication by $e(i)$ projects any $H_{d}^{p, q}$-module onto its $\boldsymbol{i}$-weight space, that is, the simultaneous generalised eigenspace for the commuting operators $x_{1}, \ldots, x_{d}$ and eigenvalues $i_{1}, \ldots, i_{d}$, respectively. All but finitely many of the $e(\boldsymbol{i})$ 's are zero, and the non-zero ones give a system of mutually orthogonal idempotents in $H_{d}^{p, q}$ summing to 1 ; see e.g. [BK2, \$3.1].

The action of the idempotent $e(i)$ on the module $\mathcal{V}\left(\lambda_{p, q}\right) \otimes V^{\otimes d}$ can be interpreted as follows. In view of Corollary 2.9, we have

$$
\mathcal{V}\left(\lambda_{p, q}\right) \otimes V^{\otimes d}=\bigoplus_{i \in \mathbb{Z}^{d}} \mathcal{F}_{i} \mathcal{V}\left(\lambda_{p, q}\right)
$$

where $\mathcal{F}_{\boldsymbol{i}}$ denotes the composite $\mathcal{F}_{i_{d}} \circ \ldots \circ \mathcal{F}_{i_{1}}$ of the functors from (2.13). By Lemma 2.10 and the definition of the actions of $x_{1}, \ldots, x_{d}$, the summand $\mathcal{F}_{i} \mathcal{V}\left(\lambda_{p, q}\right)$ in this decomposition is precisely the $i$-weight space of $\mathcal{V}\left(\lambda_{p, q}\right) \otimes V^{\otimes d}$. Hence the weight idempotent $e(\boldsymbol{i})$ acts on $\mathcal{V}\left(\lambda_{p, q}\right) \otimes V^{\otimes d}$ as the projection onto the summand $\mathcal{F}_{\boldsymbol{i}} \mathcal{V}\left(\lambda_{p, q}\right)$ along the decomposition (3.6).

Recalling the interval $I_{p, q}$ from (2.6), we will usually restrict our attention to the summand

$$
T_{d}^{p, q}:=\bigoplus_{\boldsymbol{i} \in\left(I_{p, q}\right)^{d}} \mathcal{F}_{\boldsymbol{i}} \mathcal{V}\left(\lambda_{p, q}\right)
$$

of $\mathcal{V}\left(\lambda_{p, q}\right) \otimes V^{\otimes d}$. By the discussion in the previous paragraph, we see that equivalently 


$$
\begin{aligned}
T_{d}^{p, q}=\left(\mathcal{V}\left(\lambda_{p, q}\right) \otimes V^{\otimes d}\right) 1_{d}^{p, q} \text { where } & \\
1_{d}^{p, q} & :=\sum_{\boldsymbol{i} \in\left(I_{p, q}\right)^{d}} e(\boldsymbol{i}) \in H_{d}^{p, q} .
\end{aligned}
$$

As a consequence of the fact that any symmetric polynomial in $x_{1}, \ldots, x_{d}$ is central in $H_{d}$, the idempotent $1_{d}^{p, q}$ is central in $H_{d}^{p, q}$. The space $T_{d}^{p, q}$ is naturally a right module over $1_{d}^{p, q} H_{d}^{p, q}$, which is a sum of blocks of $H_{d}^{p, q}$. Hence the map $\Phi$ from (3.5) induces an algebra homomorphism

$$
1_{d}^{p, q} H_{d}^{p, q} \rightarrow \operatorname{End}_{G}\left(T_{d}^{p, q}\right)^{\mathrm{op}} .
$$

As a refinement of the surjectivity of $\Phi$ proved below, we will also see later in the section that the induced map (3.9) is an isomorphism. Note that from (3.16) onwards we will denote the algebra $1_{d}^{p, q} H_{d}^{p, q}$ instead by $R_{d}^{p, q}$.

\section{Stretched diagrams}

In this subsection, we develop some combinatorial tools which will be used initially to compute the dimension of the various endomorphism algebras that we are interested in. We say that a tuple $i \in \mathbb{Z}^{d}$ is $(p, q)$-admissible if $i_{r}$ is $\Gamma_{r-1}$-admissible for each $r=1, \ldots, d$, where $\Gamma_{0}, \ldots, \Gamma_{d}$ are defined recursively from $\Gamma_{0}:=\left\{\lambda_{p, q}\right\}$ and $\Gamma_{r}:=$ $\Gamma_{r-1}-\alpha_{i_{r}}$, with notation as in (2.2). We refer to the sequence $\mathbf{o}:=\Gamma_{d} \cdots \Gamma_{1} \Gamma_{0}$ of blocks here as the associated block sequence. The composite matching $t=t_{d} \cdots t_{1}$ defined by setting $t_{r}:=t_{i_{r}}\left(\Gamma_{r-1}\right)$ for each $r$ is the associated composite matching. Both of these things make sense only if $i \in \mathbb{Z}^{d}$ is $(p, q)$-admissible.

Lemma 3.5. If $\boldsymbol{i} \in \mathbb{Z}^{d}$ is not $(p, q)$-admissible then $\mathcal{F}_{\boldsymbol{i}} \mathcal{V}\left(\lambda_{p, q}\right)$ is zero.

Proof. This follows from the definitions and (2.13).

By a stretched cap diagram $t=t_{d} \cdots t_{1}$ of height $d$, we mean the associated composite matching for some $(p, q)$-admissible sequence $i \in \mathbb{Z}^{d}$. We can uniquely recover the sequence $\boldsymbol{i}$, hence also the associated block sequence $\mathbf{o}$, from the stretched cap diagram $\boldsymbol{t}$. Here is an example of a stretched cap diagram of height 5 , taking $m=2, n=1$ and $q-p=1$; we draw only the strip containing the vertices indexed by $I_{p, q}^{+}$, as the picture outside of this strip consists only of vertical lines, and also label the horizontal number lines by the associated block sequence $\mathbf{o}=\Gamma_{5} \cdots \Gamma_{0}$.

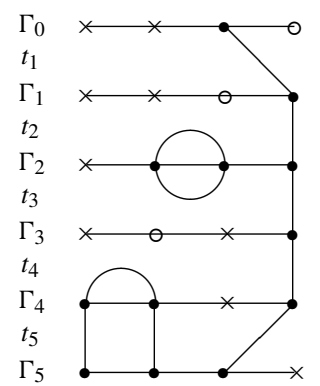


By a generalised cap in a stretched cap diagram we mean a component that meets the bottom number line at two different vertices. An oriented stretched cap diagram is a consistently oriented diagram of the form

$$
\boldsymbol{t}[\gamma]=\gamma_{d} t_{d} \gamma_{d-1} \cdots \gamma_{1} t_{1} \gamma_{0}
$$

where $\gamma=\gamma_{d} \cdots \gamma_{0}$ is a sequence of weights chosen from the associated block sequence $\mathbf{o}=\Gamma_{d} \cdots \Gamma_{0}$, i.e. $\gamma_{r} \in \Gamma_{r}$ for each $r=0, \ldots, d$. In other words, we decorate the number lines of $\boldsymbol{t}$ by weights from the appropriate blocks, in such a way that the resulting diagram is consistently oriented. (For a precise definition of the term oriented we refer to $[\mathrm{BS} 1, \S 2]$.)

Theorem 3.6. There are G-module isomorphisms

$$
\begin{aligned}
\mathcal{V}\left(\lambda_{p, q}\right) \otimes V^{\otimes d} & \cong \bigoplus_{\lambda \in \Lambda, \operatorname{ht}(\lambda)=\operatorname{ht}\left(\lambda_{p, q}\right)+d} \mathcal{P}(\lambda)^{\oplus \operatorname{dim}_{p, q}(\lambda)}, \\
T_{d}^{p, q} \cong & \bigoplus_{\lambda \in \Lambda_{p, q}, \operatorname{ht}(\lambda)=\mathrm{ht}\left(\lambda_{p, q}\right)+d} \mathcal{P}(\lambda)^{\oplus \operatorname{dim}_{p, q}(\lambda)},
\end{aligned}
$$

where $\operatorname{dim}_{p, q}(\lambda)$ is the number of oriented stretched cap diagrams $\boldsymbol{t}[\gamma]$ of height $d$ such that $\gamma_{0}=\lambda_{p, q}, \gamma_{d}=\lambda$, and all generalised caps are anti-clockwise.

Proof. For the first isomorphism, in view of Theorem 2.14 and Corollary 2.9, it suffices to prove the analogous statement on the diagram algebra side, namely, that

$$
\bigoplus_{i \in \mathbb{Z}^{d}} F_{i} V\left(\lambda_{p, q}\right) \cong \bigoplus_{\lambda \in \Lambda, \operatorname{ht}(\lambda)=\operatorname{ht}\left(\lambda_{p, q}\right)+d} P(\lambda)^{\oplus \operatorname{dim}_{p, q}(\lambda)}
$$

as $K$-modules. Remembering that $V\left(\lambda_{p, q}\right)=P\left(\lambda_{p, q}\right)$, this follows as an application of [BS2, Theorem 4.2], first using [BS2, Theorems 3.5 and 3.6] to write the composite projective functor $F_{i}=F_{i_{d}} \circ \cdots \circ F_{i_{1}}$ in terms of indecomposable projective functors.

The proof of the second isomorphism is similar, taking only $i \in\left(I_{p, q}\right)^{d}$ in (3.10). It is helpful to note that if $\lambda \in \Lambda_{p, q}$ and $t[\gamma]$ is one of the oriented stretched cap diagrams counted by $\operatorname{dim}_{p, q}(\lambda)$ then $t[\gamma]$ is trivial outside the strip containing the vertices indexed by $I_{p, q}^{+}$, i.e. it consists only of straight lines oriented $\wedge$ outside that region. This follows by considering (2.2).

Corollary 3.7. The modules $\left\{\mathcal{P}(\lambda) \mid \lambda \in \Lambda_{p, q}^{\circ}, \mathrm{ht}(\lambda)=\mathrm{ht}\left(\lambda_{p, q}\right)+d\right\}$ give a complete set of representatives for the isomorphism classes of indecomposable direct summands of $T_{d}^{p, q}$.

Proof. Suppose we are given $\lambda \in \Lambda_{p, q}^{\circ}$ with $\mathrm{ht}(\lambda)=\operatorname{ht}\left(\lambda_{p, q}\right)+d$. By Corollary 2.13, there is a sequence $\boldsymbol{i}=\left(i_{1}, \ldots, i_{d}\right) \in\left(I_{p, q}\right)^{d}$ such that $\mathcal{P}(\lambda)$ is a summand of $\mathcal{F}_{\boldsymbol{i}} \mathcal{V}\left(\lambda_{p, q}\right)$. Hence $\mathcal{P}(\lambda)$ is a summand of $T_{d}^{p, q}$. Conversely, applying Theorem 3.6, we take $\lambda \in \Lambda_{p, q}$ with $\operatorname{ht}(\lambda)=\operatorname{ht}\left(\lambda_{p, q}\right)+d$ and $\operatorname{dim}_{p, q}(\lambda) \neq 0$, and must show that $\lambda \in \Lambda_{p, q}^{\circ}$. There exists an oriented stretched cap diagram $t[\gamma]$ of height $d$ with $\gamma_{0}=\lambda_{p, q}$ and $\gamma_{d}=\lambda$, all of whose generalised caps are anti-clockwise. Every vertex labelled $\vee$ in $\lambda$ must be at 
the left end of one of these anti-clockwise generalised caps, the right end of which gives a vertex labelled $\wedge$ indexed by an integer $\leq q+n$. Recalling the definition (2.9), these observations prove that $\lambda \in \Lambda_{p, q}^{\circ}$.

Corollary 3.8. $T_{d}^{p, q}=\{0\}$ for $d>(m+n)(q-p)+2 m n$.

Proof. The set $\Lambda_{p, q}$ has a unique element $\mu_{p, q}$ of maximal height, namely, the weight

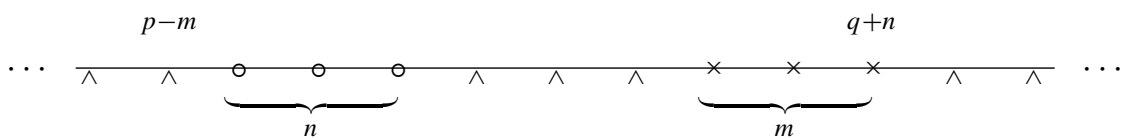

Using this and Theorem 3.6, we deduce that $T_{d}^{p, q}=\{0\}$ for $d>\operatorname{ht}\left(\mu_{p, q}\right)-\operatorname{ht}\left(\lambda_{p, q}\right)=$ $(m+n)(q-p)+2 m n$.

The mirror image of the oriented stretched cap diagram $\boldsymbol{u}[\boldsymbol{\delta}]$ in a horizontal axis is denoted $\boldsymbol{u}^{*}\left[\boldsymbol{\delta}^{*}\right]$. We call it an oriented stretched cup diagram. Then an oriented stretched circle diagram of height $d$ means a composite diagram of the form

$$
\boldsymbol{u}^{*}\left[\boldsymbol{\delta}^{*}\right] 2 \boldsymbol{t}[\gamma]=\delta_{0} u_{1}^{*} \delta_{1} \cdots \delta_{d-1} u_{d}^{*} \gamma_{d} t_{d} \gamma_{d-1} \cdots \gamma_{1} t_{1} \gamma_{0}
$$

where $t[\gamma]$ and $\boldsymbol{u}[\boldsymbol{\delta}]$ are oriented stretched cap diagrams of height $d$ with $\gamma_{d}=\delta_{d}$; see [BS3, (6.17)] for an example.

Theorem 3.9. The dimension of the algebra $\operatorname{End}_{G}\left(T_{d}^{p, q}\right)^{\mathrm{op}}$ is equal to the number of oriented stretched circle diagrams $\boldsymbol{u}^{*}\left[\delta^{*}\right] 2 \boldsymbol{t}[\gamma]$ of height d such that $\gamma_{0}=\delta_{0}=\lambda_{p, q}$ and $\gamma_{d}=\delta_{d} \in \Lambda_{p, q}$.

Proof. Applying Theorem 3.6, we see that the dimension of the endomorphism algebra is equal to

$$
\sum_{\lambda, \mu \in \Lambda_{p, q}, \operatorname{ht}(\lambda)=\operatorname{ht}(\mu)=\operatorname{ht}\left(\lambda_{p, q}\right)+d} \operatorname{dim}_{p, q}(\lambda) \cdot \operatorname{dim}_{p, q}(\mu) \cdot \operatorname{dim}_{\operatorname{Hom}_{G}}(\mathcal{P}(\lambda), \mathcal{P}(\mu)) .
$$

Also in view of Theorem 2.14, $\operatorname{dim}_{\operatorname{Hom}}(\mathcal{P}(\lambda), \mathcal{P}(\mu))=[\mathcal{P}(\mu): \mathcal{L}(\lambda)]$ is equal to the analogous dimension $\operatorname{dim}_{H_{K}}(P(\lambda), P(\mu))=[P(\mu): L(\lambda)]$ on the diagram algebra side, which is described explicitly by [BS1, (5.9)]. We deduce that $\operatorname{dim} \operatorname{Hom}_{G}(\mathcal{P}(\lambda), \mathcal{P}(\mu))$ is equal to the number of weights $v$ such that $\lambda \sim v \sim \mu$ and the circle diagram $\underline{\lambda} v \bar{\mu}$ is consistently oriented. The theorem follows easily on combining this with the combinatorial definitions of $\operatorname{dim}_{p, q}(\lambda)$ and $\operatorname{dim}_{p, q}(\mu)$ from Theorem 3.6.

The algebra $R_{d}^{p, q}$ and the isomorphism theorem

Now we need to recall some of the main results of [BS3] which give an alternative diagrammatic description of the algebra $1_{d}^{p, q} H_{d}^{p, q}$. This will allow us to see, to start with, that this algebra has the same dimension as the endomorphism algebra from Theorem 3.9. For the reader wishing to understand already the relationship between the diagram algebra $R_{d}^{p, q}$ defined in the next paragraph and the algebra $K(m \mid n)$ from the introduction, 
we point to Lemma 4.1, (4.7) and Corollary 4.6 in the next section. On the other hand Corollary 3.22 established later in this section explains the connection between $R_{d}^{p, q}$ and the representation theory of $G$.

Let $R_{d}^{p, q}$ be the associative, unital algebra with basis

$$
\left\{\begin{array}{l|l}
\left|\boldsymbol{u}^{*}\left[\boldsymbol{\delta}^{*}\right]<\boldsymbol{t}[\gamma]\right| & \begin{array}{l}
\text { for all oriented stretched circle diagrams } \boldsymbol{u}^{*}\left[\boldsymbol{\delta}^{*}\right] \imath \boldsymbol{t}[\gamma] \\
\text { of height } d \text { with } \gamma_{0}=\delta_{0}=\lambda_{p, q} \text { and } \gamma_{d}=\delta_{d} \in \Lambda_{p, q}
\end{array}
\end{array}\right\} .
$$

The multiplication is defined by an explicit algorithm described in detail in [BS3]. Briefly, to multiply two basis vectors $\left|\boldsymbol{s}^{*}\left[\boldsymbol{\tau}^{*}\right] 2 \boldsymbol{r}[\boldsymbol{\sigma}]\right|$ and $\left|\boldsymbol{u}^{*}\left[\boldsymbol{\delta}^{*}\right] 2 \boldsymbol{t}[\gamma]\right|$, the product is zero unless $\boldsymbol{r}=\boldsymbol{u}$ and all mirror image pairs of internal circles in $\boldsymbol{r}[\boldsymbol{\sigma}]$ and $\boldsymbol{u}^{*}\left[\boldsymbol{\delta}^{*}\right]$ are oriented so that one is clockwise, the other anti-clockwise. Assuming these conditions hold, the product is computed by putting $\boldsymbol{s}^{*}\left[\boldsymbol{\tau}^{*}\right]<\boldsymbol{r}[\boldsymbol{\sigma}]$ underneath $\boldsymbol{u}^{*}\left[\boldsymbol{\delta}^{*}\right] 2 \boldsymbol{t}[\gamma]$, erasing all internal circles and number lines in $\boldsymbol{r}[\boldsymbol{\sigma}]$ and $\boldsymbol{u}^{*}\left[\boldsymbol{\delta}^{*}\right]$, then iterating the generalised surgery procedure to smooth out the symmetric middle section of the diagram.

Lemma 3.10. The algebras $R_{d}^{p, q}$ and $\operatorname{End}_{G}\left(T_{d}^{p, q}\right)^{\text {op }}$ have the same dimension. In particular, $R_{d}^{p, q}$ is the zero algebra for $d>(m+n)(q-p)+2 m n$.

Proof. The number of elements in the diagram basis for $R_{d}^{p, q}$ is the same as the dimension of the algebra $\operatorname{End}_{G}\left(T_{d}^{p, q}\right)^{\text {op }}$ thanks to Theorem 3.9. The last statement follows from Corollary 3.8.

As a consequence of [BS3, Corollary 8.6], we can identify $R_{d}^{p, q}$ with a certain cyclotomic Khovanov-Lauda-Rouquier algebra in the sense of [KL, Ro]. To make this identification explicit, we need to define some special elements

$$
\left\{e(i) \mid i \in\left(I_{p, q}\right)^{d}\right\} \cup\left\{y_{1}, \ldots, y_{d}\right\} \cup\left\{\psi_{1}, \ldots, \psi_{d-1}\right\}
$$

in $R_{d}^{p, q}$. For $\boldsymbol{i} \in\left(I_{p, q}\right)^{d}$, we let $e(i) \in R_{d}^{p, q}$ be the idempotent defined as follows. If $\boldsymbol{i}$ is not $(p, q)$-admissible then $e(i):=0$. If it is admissible, let $t=t_{d} \cdots t_{1}$ be the associated composite matching and $\mathbf{o}=\Gamma_{d} \cdots \Gamma_{0}$ be the associated block sequence. Then

$$
e(i):=\sum_{\delta, \gamma}\left|t^{*}\left[\delta^{*}\right] \imath t[\gamma]\right|
$$

where the sum is over all sequences $\gamma=\gamma_{d} \cdots \gamma_{0}$ and $\delta=\delta_{d} \cdots \delta_{0}$ of weights with each $\gamma_{r}, \delta_{r} \in \Gamma_{r}$ chosen so that every circle of $\boldsymbol{t}^{*}\left[\boldsymbol{\delta}^{*}\right]$ 乙 $\boldsymbol{t}[\gamma]$ crossing the middle number line is anti-clockwise, and all remaining mirror image pairs of circles are oriented so that one is clockwise, the other anti-clockwise. The elements $\left\{e(\boldsymbol{i}) \mid \boldsymbol{i} \in\left(I_{p, q}\right)^{d}\right\}$ give a system of mutually orthogonal idempotents whose sum is the identity in $R_{d}^{p, q}$.

Next we define the elements $y_{1}, \ldots, y_{d}$. Let $\bar{y}_{1}, \ldots, \bar{y}_{d}$ be the unique elements of $R_{d}^{p, q}$ such that the product $\left|\boldsymbol{u}^{*}\left[\boldsymbol{\delta}^{*}\right] \imath \boldsymbol{t}[\gamma]\right| \cdot \bar{y}_{r}$ (resp. $\left.\bar{y}_{r} \cdot\left|\boldsymbol{u}^{*}\left[\boldsymbol{\delta}^{*}\right] \imath \boldsymbol{t}[\boldsymbol{\gamma}]\right|\right)$ is computed by making a positive circle move in the section of $\boldsymbol{u}^{*} \boldsymbol{t}$ containing $t_{r}$ (resp. $u_{r}^{*}$ ), as described in detail in $[\mathrm{BS} 3,(5.5),(5.11)]$. Also introduce the signs

$$
\sigma_{p, q}^{r}(\boldsymbol{i}):=(-1)^{\min \left(p, i_{r}\right)+\min \left(q, i_{r}\right)+m-p-\delta_{i_{1}, i_{r}}-\cdots-\delta_{i_{r-1}, i_{r}}} .
$$


Then we define $y_{r}:=\sum_{\boldsymbol{i} \in\left(I_{p, q}\right)^{d}} y_{r} e(\boldsymbol{i})$ where

$$
y_{r} e(i):=\sigma_{p, q}^{r}(i) \bar{y}_{r} e(i),
$$

to get the elements $y_{r} \in R_{d}^{p, q}$ for $r=1, \ldots, d$.

Finally we define $\psi_{1}, \ldots, \psi_{d-1}$. Let $\bar{\psi}_{1}, \ldots, \bar{\psi}_{d-1}$ be the unique elements of $R_{d}^{p, q}$ such that the product $\mid \boldsymbol{u}^{*}\left[\boldsymbol{\delta}^{*}\right]\left\llcorner\boldsymbol{t}[\gamma] \mid \cdot \bar{\psi}_{r}\right.$ (resp. $\bar{\psi}_{r} \cdot \mid \boldsymbol{u}^{*}\left[\boldsymbol{\delta}^{*}\right]\llcorner\boldsymbol{t}[\gamma] \mid$ ) is computed by making a negative circle move, a crossing move or a height move in the section of $\boldsymbol{u}^{*} \boldsymbol{t}$ containing $t_{r+1} t_{r}$ (resp. $u_{r}^{*} u_{r+1}^{*}$ ), as described in detail in [BS3, (5.7), (5.12)]. Then we define $\psi_{r}:=\sum_{i \in\left(I_{p, q}\right)^{d}} \psi_{r} e(i)$ where

$$
\psi_{r} e(\boldsymbol{i}):= \begin{cases}-\sigma_{p, q}^{r}(i) \bar{\psi}_{r} e(i) & \text { if } i_{r+1}=i_{r} \text { or } i_{r+1}=i_{r}+1 \\ \bar{\psi}_{r} e(i) & \text { otherwise }\end{cases}
$$

to get the elements $\psi_{r} \in R_{d}^{p, q}$ for $r=1, \ldots, d-1$.

Theorem 3.11. The elements (3.11) generate $R_{d}^{p, q}$ subject only to the defining relations of the Khovanov-Lauda-Rouquier algebra associated to the linear quiver

with vertices indexed by the set $I_{p, q}$ in order from left to right (see e.g. [BS3, (6.8)(6.16)]), plus the additional cyclotomic relations $y_{1}^{\delta_{i_{1}, p}+\delta_{i_{1}, q}} e(\boldsymbol{i})=0$ for $\boldsymbol{i}=\left(i_{1}, \ldots, i_{d}\right)$ $\in\left(I_{p, q}\right)^{d}$.

Proof. This is a consequence of [BS3, Corollary 8.6]. More precisely, we apply [BS3, Corollary 8.6], taking the index set $I$ there to be the set $I_{p, q}$, the pair $(o+m, o+n)$ there to be $(p, q)$, and summing over all $\alpha \in Q_{+}$of height $d$. This implies that the given quotient of the Khovanov-Lauda-Rouquier algebra is isomorphic to the diagram algebra with basis consisting of oriented stretched circle diagrams $\mid \boldsymbol{u}^{*}\left[\boldsymbol{\delta}^{*}\right]$ z $\boldsymbol{t}[\boldsymbol{\gamma}] \mid$ just like the ones considered here, except they are drawn only in the strip containing the vertices indexed by $I_{p, q}^{+}$. The isomorphism in [BS3] is not quite the same as the map here, because the sign in (3.13) differs from the corresponding sign chosen in [BS3] by a factor of $(-1)^{m-p}$; this causes no problems as it amounts to twisting by an automorphism of the Khovanov-Lauda-Rouquier algebra. It remains to observe that all the oriented stretched circle diagrams in the statement of the present theorem are trivial outside the strip $I_{p, q}^{+}$, consisting only of straight lines oriented $\wedge$ in that region; these have no effect on the multiplication.

Now we can formulate the following key isomorphism theorem, which identifies the algebras $1_{d}^{p, q} H_{d}^{p, q}$ and $R_{d}^{p, q}$.

Theorem 3.12. There is a unique algebra isomorphism

$$
1_{d}^{p, q} H_{d}^{p, q} \stackrel{\sim}{\rightarrow} R_{d}^{p, q}
$$


such that $e(\boldsymbol{i}) \mapsto e(\boldsymbol{i}), x_{r} e(\boldsymbol{i}) \mapsto\left(y_{r}+i_{r}\right) e(\boldsymbol{i})$ and $s_{r} e(\boldsymbol{i}) \mapsto\left(\psi_{r} q_{r}(\boldsymbol{i})-p_{r}(\boldsymbol{i})\right) e(\boldsymbol{i})$ for each $r$ and $i \in\left(I_{p, q}\right)^{d}$, where $p_{r}(i), q_{r}(i) \in R_{d}^{p, q}$ are chosen as in [BK2, §3.3], e.g. one could take

$$
\begin{aligned}
& p_{r}(\boldsymbol{i}):= \begin{cases}1 & \text { if } i_{r}=i_{r+1}, \\
-\left(i_{r+1}-i_{r}+y_{r+1}-y_{r}\right)^{-1} & \text { if } i_{r} \neq i_{r+1} ;\end{cases} \\
& q_{r}(\boldsymbol{i}):= \begin{cases}1+y_{r+1}-y_{r} & \text { if } i_{r}=i_{r+1}, \\
\left(2+y_{r+1}-y_{r}\right)\left(1+y_{r+1}-y_{r}\right)^{-2} & \text { if } i_{r+1}=i_{r}+1, \\
1 & \text { if } i_{r+1}=i_{r}-1, \\
1+\left(i_{r+1}-i_{r}+y_{r+1}-y_{r}\right)^{-1} & \text { if }\left|i_{r}-i_{r+1}\right|>1 .\end{cases}
\end{aligned}
$$

(The inverses on the right hand sides of these formulae make sense because each $y_{r+1}-y_{r}$ is nilpotent with nilpotency degree at most two, as is clear from the diagrammatic definition of the $y_{r}$ 's.)

Proof. This is a consequence of Theorem 3.11 combined with the main theorem of [BK2]; see also [BS3, Theorem 8.5].

Henceforth, we will use the isomorphism from the above theorem to identify the algebra $1_{d}^{p, q} H_{d}^{p, q}$ with $R_{d}^{p, q}$, so

$$
1_{d}^{p, q} H_{d}^{p, q} \equiv R_{d}^{p, q}
$$

We will denote it always by the more compact notation $R_{d}^{p, q}$. Thus there are three different ways of viewing $R_{d}^{p, q}$ : it is a diagram algebra with basis given by oriented stretched circle diagrams, it is a cyclotomic Khovanov-Lauda-Rouquier algebra, and it is a sum of blocks of the cyclotomic Hecke algebra $H_{d}^{p, q}$.

\section{Super version of level two Schur-Weyl duality}

Now we can prove the main results of the section, namely, that the map $\Phi$ from (3.5) is surjective and the induced map (3.9) is an isomorphism. In the case $d \leq \min (m, n)$ we have already done most of the work:

Theorem 3.13. If $d \leq \min (m, n)$ then $T_{d}^{p, q}=\mathcal{V}\left(\lambda_{p, q}\right) \otimes V^{\otimes d}, R_{d}^{p, q}=H_{d}^{p, q}$, and the map

$$
\Phi: H_{d}^{p, q} \rightarrow \operatorname{End}_{G}\left(\mathcal{V}\left(\lambda_{p, q}\right) \otimes V^{\otimes d}\right)^{\mathrm{op}}
$$

is an algebra isomorphism.

Proof. Let us first show that $T_{d}^{p, q}=\mathcal{V}\left(\lambda_{p, q}\right) \otimes V^{\otimes d}$. Observe for $d \leq \min (m, n)$ that any $(p, q)$-admissible sequence $i \in \mathbb{Z}^{d}$ necessarily lies in $\left(I_{p, q}\right)^{d}$. This is clear from (2.2) and the form of the diagram $\lambda_{p, q}$. Hence applying Lemma 3.5 we see that $\mathcal{F}_{\boldsymbol{i}} \mathcal{V}\left(\lambda_{p, q}\right)=\{0\}$ for $i \in \mathbb{Z}^{d} \backslash\left(I_{p, q}\right)^{d}$. So we are done by (3.7).

Now consider the map $\Phi$. It is injective by Corollary 3.4. To show that it is an isomorphism, we apply Lemma 3.10, recalling the identification (3.16), to see that

$$
\operatorname{dim} \operatorname{End}_{G}\left(\mathcal{V}\left(\lambda_{p, q}\right) \otimes V^{\otimes d}\right)^{\mathrm{op}}=\operatorname{dim}_{\operatorname{End}}\left(T_{d}^{p, q}\right)^{\mathrm{op}}=\operatorname{dim} R_{d}^{p, q} \leq \operatorname{dim} H_{d}^{p, q} .
$$


Hence our injective map is an isomorphism. At the same time, we deduce that $\operatorname{dim} R_{d}^{p, q}=$ $\operatorname{dim} H_{d}^{p, q}$, hence $R_{d}^{p, q}=H_{d}^{p, q}$.

It remains to consider the cases with $d>\min (m, n)$. For that, following a standard argument, we need to allow $m$ and $n$ to vary. So we take some other integers $m^{\prime}, n^{\prime} \geq 0$ and consider the supergroup $G^{\prime}=G L\left(m^{\prime} \mid n^{\prime}\right)$. To avoid any confusion, we decorate all notation related to $G^{\prime}$ with a prime, e.g. $V^{\prime}$ is its natural module, its irreducible modules are the modules denoted $\mathcal{L}^{\prime}(\lambda)$ for $\lambda \in \Lambda^{\prime}:=\Lambda\left(m^{\prime} \mid n^{\prime}\right)$, and $\mathscr{F}^{\prime}:=\mathscr{F}\left(m^{\prime} \mid n^{\prime}\right)$. We are going to exploit the following standard lemma.

Lemma 3.14. Let $F: \mathscr{F}^{\prime} \rightarrow \mathscr{F}$ be an exact functor and $X \subseteq \Lambda^{\prime}$ be a subset with the following properties:

(i) $F$ commutes with duality, i.e. $F \circ \circledast \cong \circledast \circ F$;

(ii) the modules $F \mathcal{V}^{\prime}(\lambda)$ for $\lambda \in X$ have standard flags;

(iii) the map $\operatorname{Hom}_{G^{\prime}}\left(\mathcal{V}^{\prime}(\lambda), \mathcal{V}^{\prime}(\mu)^{\circledast}\right) \rightarrow \operatorname{Hom}_{G}\left(F \mathcal{V}^{\prime}(\lambda), F \mathcal{V}^{\prime}(\mu)^{\circledast}\right)$ defined by the functor $F$ is surjective for all $\lambda, \mu \in X$.

Suppose $M, N$ are $G^{\prime}$-modules with standard flags all of whose sections are of the form $\mathcal{V}^{\prime}(\lambda)$ for $\lambda \in X$. Then the map $\operatorname{Hom}_{G^{\prime}}\left(M, N^{\circledast}\right) \rightarrow \operatorname{Hom}_{G}\left(F M, F N^{\circledast}\right)$ defined by the functor $F$ is surjective.

Proof. We proceed by induction on the sum of the lengths of the standard flags of $M$ and $N$, the base case being covered by (iii). For the induction step, either $M$ or $N$ has a standard flag of length greater than one. It suffices to consider the case when the standard flag of $M$ has length greater than 1, since the other case reduces to that using duality. Pick a submodule $K$ of $M$ such that both $K$ and $Q:=M / K$ are non-zero and possess standard flags. By a general fact about highest weight categories (see also [B2, Lemma 3.6] for a short direct proof in this context), the functor $\operatorname{Hom}_{G^{\prime}}\left(?, N^{\circledast}\right)$ is exact on sequences of $G^{\prime}$-modules possessing a standard flag. So applying it to the short exact sequence $0 \rightarrow K \rightarrow M \rightarrow Q \rightarrow 0$ we get a short exact sequence as on the top line of the following diagram:

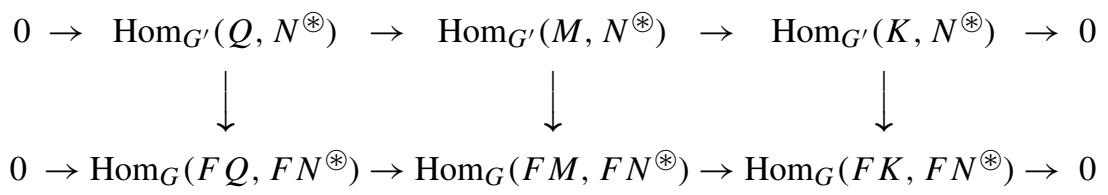

Similar considerations applying the functor $\operatorname{Hom}_{G}\left(\right.$ ?,$\left.F N^{\circledast}\right)$ to $0 \rightarrow F K \rightarrow F M \rightarrow$ $F Q \rightarrow 0$ gives the short exact sequence in the bottom row. The vertical maps making the diagram commute are the maps defined by the functor $F$. The left and right vertical arrows are surjective by the induction hypothesis. Hence the middle vertical arrow is surjective too by the five lemma.

Now we consider the situation that

$$
G^{\prime}=G L(m \mid n+1) .
$$


Embed $G=G L(m \mid n)$ into $G^{\prime}$ in the top left hand corner in the obvious way. Also let $S$ be the one-dimensional torus embedded into $G^{\prime}$ in the bottom right hand corner, so that $S$ centralises the subgroup $G$. The character group $X(S)$ is generated by $\varepsilon_{m+n+1}$. Let $F_{+}: \mathscr{F}^{\prime} \rightarrow \mathscr{F}$ be the functor mapping $M \in \mathscr{F}^{\prime}$ to the $-(q+m) \varepsilon_{m+n+1}$-weight space of $\Pi^{q+m} M$ with respect to the torus $S$.

Lemma 3.15. Let $G^{\prime}=G L(m \mid n+1)$ and $G=G L(m \mid n)$ as in (3.17).

(i) For $\lambda \in X^{+}\left(T^{\prime}\right)$ with $\left(\lambda, \varepsilon_{m+n+1}\right)=q+m$, we have $F_{+} \mathcal{V}^{\prime}(\lambda) \cong \mathcal{V}(\mu)$, where $\mu$ is the restriction of $\lambda$ to $T<T^{\prime}$.

(ii) $F_{+} \mathcal{V}^{\prime}\left(\lambda_{p, q}^{\prime}\right) \cong \mathcal{V}\left(\lambda_{p, q}\right)$.

(iii) For $\lambda \in X^{+}(T)$ with $\left(\lambda, \varepsilon_{m+n+1}\right)<q+m$, we have $F_{+} \mathcal{V}^{\prime}(\lambda)=\{0\}$.

Proof. The proof of (i) reduces easily using the definition (2.10) to checking that the $-(q+m) \varepsilon_{m+n+1}$-weight space of $\Pi^{q+m} E^{\prime}(\lambda)$ with respect to $S$ is isomorphic to $E(\mu)$ as a $G_{0}$-module, which is well known. Then (ii) is a consequence of (i), noting that $\lambda_{p, q}^{\prime}=$ $\sum_{r=1}^{m} p \varepsilon_{r}-\sum_{s=1}^{n+1}(q+m) \varepsilon_{m+s}$. The proof of (iii) is similar to (i).

Lemma 3.16. Let $G^{\prime}=G L(m \mid n+1)$ and $G=G L(m \mid n)$ as in (3.17). There is a unique G-module isomorphism

$$
F_{+}\left(\mathcal{V}^{\prime}\left(\lambda_{p, q}^{\prime}\right) \otimes\left(V^{\prime}\right)^{\otimes d}\right) \stackrel{\sim}{\rightarrow} \mathcal{V}\left(\lambda_{p, q}\right) \otimes V^{\otimes d}
$$

such that $v_{p, q}^{\prime} \otimes v_{i_{1}}^{\prime} \otimes \cdots \otimes v_{i_{d}}^{\prime} \mapsto v_{p, q} \otimes v_{i_{1}} \otimes \cdots \otimes v_{i_{d}}$ for all $1 \leq i_{1}, \ldots, i_{d} \leq m+n$. Moreover, this map intertwines the natural actions of $H_{d}^{p, q}$.

Proof. The first statement follows using the isomorphism $F_{+} \mathcal{V}^{\prime}\left(\lambda_{p, q}^{\prime}\right) \cong \mathcal{V}\left(\lambda_{p, q}\right)$ from the first part of the previous lemma, together with the following observations:

- the weights $\mu$ arising with non-zero multiplicity in $\mathcal{V}^{\prime}\left(\lambda_{p, q}^{\prime}\right)$ all satisfy $\left(\mu, \varepsilon_{m+n+1}\right) \leq$ $q+m$;

- the weights $\mu$ arising with non-zero multiplicity in $\left(V^{\prime}\right)^{\otimes d}$ all satisfy $\left(\mu, \varepsilon_{m+n+1}\right) \leq 0$;

- the zero weight space of $\left(V^{\prime}\right)^{\otimes d}$ with respect to $S$ is $V^{\otimes d}$.

The second statement is straightforward.

Lemma 3.17. Let $G^{\prime}=G L(m \mid n+1)$ and $G=G L(m \mid n)$ as in (3.17). Assume the map $\Phi^{\prime}: H_{d}^{p, q} \rightarrow \operatorname{End}_{G^{\prime}}\left(\mathcal{V}^{\prime}\left(\lambda_{p, q}^{\prime}\right) \otimes\left(V^{\prime}\right)^{\otimes d}\right)^{\text {op }}$ is surjective. Then the map $\Phi: H_{d}^{p, q} \rightarrow$ $\operatorname{End}_{G}\left(\mathcal{V}\left(\lambda_{p, q}\right) \otimes V^{\otimes d}\right)^{\mathrm{op}}$ is surjective too.

Proof. We apply Lemma 3.14 to $F:=F_{+}$, taking $m^{\prime}:=m, n^{\prime}:=n+1$ and the set of weights $X$ to be $\left\{\lambda \in X^{+}\left(T^{\prime}\right) \mid\left(\lambda, \varepsilon_{m+n+1}\right) \leq q+m\right\}$. The hypothesis in Lemma 3.14(ii) follows from Lemma 3.15, and the other two hypotheses are clear. Since $\mathcal{V}^{\prime}\left(\lambda_{p, q}^{\prime}\right) \otimes\left(V^{\prime}\right)^{\otimes d}$ is self-dual and has a standard flag, we deduce that the functor $F_{+}$ defines a surjection

$$
\operatorname{End}_{G^{\prime}}\left(\mathcal{V}^{\prime}\left(\lambda_{p, q}^{\prime}\right) \otimes\left(V^{\prime}\right)^{\otimes d}\right)^{\mathrm{op}} \rightarrow \operatorname{End}_{G}\left(F_{+}\left(\mathcal{V}^{\prime}\left(\lambda_{p, q}^{\prime}\right) \otimes\left(V^{\prime}\right)^{\otimes d}\right)\right)^{\mathrm{op}}
$$


Composing with the isomorphism from Lemma 3.16 and using also the last part of that lemma, we deduce that there is a commutative triangle

$$
\operatorname{End}_{G^{\prime}}\left(\mathcal{V}^{\prime}\left(\lambda_{p, q}^{\prime}\right) \otimes\left(V^{\prime}\right)^{\otimes d}\right)^{\mathrm{op}} \longrightarrow{ }^{\Phi^{\prime}}{ }^{p, q}
$$

in which the bottom map is surjective. The map $\Phi^{\prime}$ is surjective by assumption. So we deduce that $\Phi$ is surjective too.

Instead consider the situation that

$$
G^{\prime}=G L(m+1 \mid n)
$$

and embed $G=G L(m \mid n)$ into $G^{\prime}$ into the bottom right hand corner in the obvious way. Also let $S$ be the one-dimensional torus embedded into $G^{\prime}$ in the top left hand corner, so again $S$ centralises the subgroup $G$. The character group $X(S)$ is generated by $\varepsilon_{1}$. Let $F_{-}: \mathscr{F}^{\prime} \rightarrow \mathscr{F}$ be the functor mapping $M \in \mathscr{F}^{\prime}$ to the $(p-n) \varepsilon_{1}$-weight space of $M$ with respect to the torus $S$. The analogues of Lemmas 3.15-3.17 in the new situation are as follows.

Lemma 3.18. Let $G^{\prime}=G L(m+1 \mid n)$ and $G=G L(m \mid n)$ as in (3.18).

(i) For $\lambda \in X^{+}\left(T^{\prime}\right)$ with $\left(\lambda, \varepsilon_{1}\right)=p$, we have $F_{-} \mathcal{V}^{\prime}(\lambda) \cong \mathcal{V}(\mu)$, where $\mu$ is the restriction of $\lambda-n \varepsilon_{1}+\varepsilon_{m+2}+\cdots+\varepsilon_{m+n+1}$ to $T$.

(ii) $F_{-} \mathcal{V}^{\prime}\left(\lambda_{p, q}^{\prime}\right) \cong \mathcal{V}\left(\lambda_{p, q}\right)$.

(iii) For $\lambda \in X^{+}\left(T^{\prime}\right)$, with $\left(\lambda, \varepsilon_{1}\right)>p$, we have $F_{-} \mathcal{V}^{\prime}(\lambda)=\{0\}$.

Proof. This follows by similar arguments to the proof of Lemma 3.15, but there is an additional subtlety. The main new point is that if $v_{+}$is a non-zero highest weight vector of weight $\lambda$ in $\mathcal{V}^{\prime}(\lambda)$ as in (i), then the vector $e_{1, m+2} \cdots e_{1, m+n+1} v_{+}$gives a highest weight vector for $G$ in $F_{-} \mathcal{V}^{\prime}(\lambda)$ of weight $\lambda-n \varepsilon_{1}+\varepsilon_{m+2}+\cdots+\varepsilon_{m+n+1}$. This statement is checked by explicit calculation in $U\left(\mathfrak{g}^{\prime}\right)$. It then follows from the PBW theorem that this vector generates $F_{-} \mathcal{V}^{\prime}(\lambda)$ and $F_{-} \mathcal{V}^{\prime}(\lambda) \cong \mathcal{V}(\mu)$ to give (i). For (ii) we note that $\lambda_{p, q}^{\prime}=\sum_{r=1}^{m+1} p \varepsilon_{r}-\sum_{s=1}^{n}(q+m+1) \varepsilon_{m+1+s}$.

Lemma 3.19. Let $G^{\prime}=G L(m+1 \mid n)$ and $G=G L(m \mid n)$ as in (3.18). There is a unique G-module isomorphism

$$
F_{-}\left(\mathcal{V}^{\prime}\left(\lambda_{p, q}^{\prime}\right) \otimes\left(V^{\prime}\right)^{\otimes d}\right) \stackrel{\sim}{\rightarrow} \mathcal{V}\left(\lambda_{p, q}\right) \otimes V^{\otimes d}
$$

such that $e_{1, m+2} \cdots e_{1, m+n+1} \cdot v_{p, q}^{\prime} \otimes v_{i_{1}}^{\prime} \otimes \cdots \otimes v_{i_{d}}^{\prime} \mapsto v_{p, q} \otimes v_{i_{1}-1} \otimes \cdots \otimes v_{i_{d}-1}$ for all $2 \leq i_{1}, \ldots, i_{d} \leq m+n+1$. Moreover, this map intertwines the natural actions of $H_{d}^{p, q}$.

Proof. Similar to the proof of Lemma 3.16, using Lemma 3.18.

Lemma 3.20. Let $G^{\prime}=G L(m+1 \mid n)$ and $G=G L(m \mid n)$ as in (3.18). Assume the map $\Phi^{\prime}: H_{d}^{p, q} \rightarrow \operatorname{End}_{G^{\prime}}\left(\mathcal{V}^{\prime}\left(\lambda_{p, q}^{\prime}\right) \otimes\left(V^{\prime}\right)^{\otimes d}\right)^{\mathrm{op}}$ is surjective. Then the map $\Phi: H_{d}^{p, q} \rightarrow$ $\operatorname{End}_{G}\left(\mathcal{V}\left(\lambda_{p, q}\right) \otimes V^{\otimes d}\right)^{\text {op }}$ is surjective too. 
Proof. Apply Lemma 3.14 taking $m^{\prime}:=m+1, n^{\prime}:=n$ and the set $X$ of weights to be $\left\{\lambda \in X^{+}\left(T^{\prime}\right) \mid\left(\lambda, \varepsilon_{1}\right) \geq p\right\}$, arguing in the same way as in the proof of Lemma 3.17.

Finally we can assemble the pieces to prove a key result, which is a super analogue of the Schur-Weyl duality for level two from [BK1].

Theorem 3.21 (Super Schur-Weyl duality). For any $d \geq 0$, the map

$$
\Phi: H_{d}^{p, q} \rightarrow \operatorname{End}_{G}\left(\mathcal{V}\left(\lambda_{p, q}\right) \otimes V^{\otimes d}\right)^{\text {op }}
$$

is surjective.

Proof. In the case that $d \leq \min (m, n)$, this is immediate from Theorem 3.13. To prove it in general, pick $m \leq m^{\prime}$ and $n \leq n^{\prime}$ so that $d \leq \min \left(m^{\prime}, n^{\prime}\right)$. We already know the surjectivity of the map $\Phi^{\prime}$ for $G^{\prime}=G L\left(m^{\prime} \mid n^{\prime}\right)$. Now apply Lemma 3.17 a total of $n^{\prime}-n$ times and Lemma 3.20 a total of $m^{\prime}-m$ times to deduce the surjectivity for $G=G L(m \mid n)$.

Corollary 3.22. Recalling the identification (3.16), the map $\Phi$ induces an algebra isomorphism $R_{d}^{p, q} \stackrel{\sim}{\rightarrow} \operatorname{End}_{G}\left(T_{d}^{p, q}\right)^{\mathrm{op}}$.

Proof. Theorem 3.21 shows the induced map $R_{d}^{p, q} \rightarrow \operatorname{End}_{G}\left(T_{d}^{p, q}\right)^{\text {op }}$ is surjective. It is an isomorphism by Lemma 3.10 .

\section{Irreducible representations of $R_{d}^{p, q}$}

As an application of Corollary 3.22, we can recover the known classification of the irreducible $R_{d}^{p, q}$-modules. For $\lambda \in \Lambda_{p, q}^{\circ}$ with $\operatorname{ht}(\lambda)=\operatorname{ht}\left(\lambda_{p, q}\right)+d$, let

$$
D^{p, q}(\lambda):=\operatorname{Hom}_{G}\left(T_{d}^{p, q}, \mathcal{L}(\lambda)\right)
$$

viewed as a left $R_{d}^{p, q}$-module in the natural way.

Theorem 3.23. The modules $\left\{D^{p, q}(\lambda) \mid \lambda \in \Lambda_{p, q}^{\circ}, \mathrm{ht}(\lambda)=\mathrm{ht}\left(\lambda_{p, q}\right)+d\right\}$ give a complete set of pairwise inequivalent irreducible $R_{d}^{p, q}$-modules. Moreover, $\operatorname{dim} D^{p, q}(\lambda)=$ $\operatorname{dim}_{p, q}(\lambda)$, where $\operatorname{dim}_{p, q}(\lambda)$ is as defined in Theorem 3.6.

Proof. As $T_{d}^{p, q}$ is a projective module, Corollary 3.22 and the usual theory of functors of the form $\operatorname{Hom}(P, ?)$ imply that the non-zero modules of the form $\operatorname{Hom}_{G}\left(T_{d}^{p, q}, \mathcal{L}(\lambda)\right)$ for $\lambda \in \Lambda$ give a complete set of pairwise inequivalent irreducible $R_{d}^{p, q}$-modules. The nonzero ones are parametrised by the weights $\lambda \in \Lambda_{p, q}^{\circ}$ with $\operatorname{ht}(\lambda)=\operatorname{ht}\left(\lambda_{p, q}\right)+d$, thanks to Corollary 3.7. Finally the stated dimension formula is a consequence of Theorem 3.6.

Remark 3.24. For a graded version of the dimension formula for the irreducible $R_{d}^{p, q}$ modules derived in Theorem 3.23, we refer the reader to [BS3, Theorem 9.9]. (The identification of the labellings of irreducible representations in the above theorem with the one in [BS3] can be deduced using the methods of the next subsection.) 


\section{$i$-Restriction and $i$-induction}

To identify the labelling of irreducible $R_{d}^{p, q}$-modules from Theorem 3.23 with other known parametrisations, it is useful to have available a more intrinsic characterisation of $D^{p, q}(\lambda)$. We explain one inductive approach to this here in terms of the well-known $i$-restriction functors.

Suppose that $i \in I_{p, q}$. The natural inclusion $H_{d} \hookrightarrow H_{d+1}$ induces an embedding $H_{d}^{p, q} \hookrightarrow H_{d+1}^{p, q}$. Composing before and after with the inclusion $R_{d}^{p, q}=1_{d}^{p, q} H_{d}^{p, q} \hookrightarrow$ $H_{d+1}^{p, q}$ and the projection $H_{d+1}^{p, q} \rightarrow 1_{d+1}^{p, q} H_{d+1}^{p, q}=R_{d+1}^{p, q}$, we get a unital algebra homomorphism

$$
\theta_{d}: R_{d}^{p, q} \rightarrow R_{d+1}^{p, q}
$$

Note this map need not be injective, e.g. if $d=(m+n)(p-q)+2 m n$ then the algebra $R_{d}^{p, q}$ is non-zero but $R_{d+1}^{p, q}$ is zero by Lemma 3.10. The image of $x_{d+1}$ in $R_{d+1}^{p, q}$ centralises $\theta_{d}\left(R_{d}^{p, q}\right)$. So it makes sense to define the $i$-restriction functor

$$
\mathcal{E}_{i}: \operatorname{rep}\left(R_{d+1}^{p, q}\right) \rightarrow \operatorname{rep}\left(R_{d}^{p, q}\right)
$$

to be the exact functor mapping an $R_{d+1}^{p, q}$-module $M$ to the generalised $i$-eigenspace of $x_{d+1}$ on $M$, viewed as an $R_{d}^{p, q}$-module via $\theta_{d}$.

For us, a slightly different formulation of this definition will be more convenient. Let

$$
1_{d ; i}^{p, q}:=\sum_{\boldsymbol{i} \in\left(I_{p, q}\right)^{d+1}, i_{d+1}=i} e(\boldsymbol{i}) \in R_{d+1}^{p, q} .
$$

Multiplication by this idempotent projects any $R_{d+1}^{p, q}$-module $M$ onto the generalised $i$ eigenspace of $x_{d+1}$, which is a module over the subring $1_{d ; i}^{p, q} R_{d+1}^{p, q} 1_{d ; i}^{p, q}$ of $R_{d+1}^{p, q}$. As $1_{d ; i}^{p, q}$ centralises the image of the homomorphism $\theta_{d}$, we can define another unital algebra homomorphism

$$
\theta_{d ; i}: R_{d}^{p, q} \rightarrow 1_{d ; i}^{p, q} R_{d+1}^{p, q} 1_{d ; i}^{p, q}, \quad x \mapsto \theta_{d}(x) 1_{d ; i}^{p, q} .
$$

Because $1_{d+1}^{p, q}=\sum_{i \in I_{p, q}} 1_{d ; i}^{p, q}$, we have $\theta_{d}=\sum_{i \in I_{p, q}} \theta_{d ; i}$. The functor $\mathcal{E}_{i}$ from the previous paragraph can be defined equivalently as the functor mapping an $R_{d+1}^{p, q}$-module $M$ to the space $1_{d ; i}^{p, q} M$ viewed as an $R_{d}^{p, q}$-module via the homomorphism $\theta_{d ; i}$. So

$$
\mathcal{E}_{i} M=1_{d ; i}^{p, q} M \cong \operatorname{Hom}_{R_{d+1}^{p, q}}\left(R_{d+1}^{p, q} 1_{d ; i}^{p, q}, M\right),
$$

where we view $R_{d+1}^{p, q} 1_{d ; i}^{p, q}$ as an $\left(R_{d+1}^{p, q}, R_{d}^{p, q}\right)$-bimodule using the homomorphism $\theta_{d ; i}$ to get the right module structure. It is clear from (3.24) that the $i$-restriction functor $\mathcal{E}_{i}$ has a left adjoint

$$
\mathcal{F}_{i}:=R_{d+1}^{p, q} 1_{d ; i}^{p, q} \otimes_{R_{d}^{p, q}} ?: \operatorname{rep}\left(R_{d}^{p, q}\right) \rightarrow \operatorname{rep}\left(R_{d+1}^{p, q}\right) .
$$

We refer to this as the $i$-induction functor. 
Lemma 3.25. There is an isomorphism

$$
r: \mathcal{E}_{i} \circ \operatorname{Hom}_{G}\left(T_{d+1}^{p, q}, ?\right) \stackrel{\sim}{\rightarrow} \operatorname{Hom}_{G}\left(T_{d}^{p, q}, ?\right) \circ \mathcal{E}_{i}
$$

of functors from $\mathscr{F}$ to $\operatorname{rep}\left(R_{d}^{p, q}\right)$.

Proof. Take $M \in \mathscr{F}$. Note recalling (3.6) that $T_{d+1}^{p, q} 1_{d ; i}^{p, q}=\mathcal{F}_{i} T_{d}^{p, q}$. So we can identify

$$
\begin{aligned}
\mathcal{E}_{i}\left(\operatorname{Hom}_{G}\left(T_{d+1}^{p, q}, M\right)\right) & =1_{d ; i}^{p, q} \operatorname{Hom}_{G}\left(T_{d+1}^{p, q}, M\right)=\operatorname{Hom}_{G}\left(T_{d+1}^{p, q} 1_{d ; i}^{p, q}, M\right) \\
& =\operatorname{Hom}_{G}\left(\mathcal{F}_{i} T_{d}^{p, q}, M\right) .
\end{aligned}
$$

Then the adjunction between $\mathcal{F}_{i}$ and $\mathcal{E}_{i}$ fixed earlier defines a natural isomorphism

$$
\operatorname{Hom}_{G}\left(\mathcal{F}_{i} T_{d}^{p, q}, M\right) \stackrel{\sim}{\rightarrow} \operatorname{Hom}_{G}\left(T_{d}^{p, q}, \mathcal{E}_{i} M\right) .
$$

Naturality gives automatically that this is an $R_{d}^{p, q}$-module homomorphism. So we have defined the desired isomorphism of functors.

Corollary 3.26. Take $\lambda \in \Lambda_{p, q}^{\circ}$ with $\mathrm{ht}(\lambda)=\operatorname{ht}\left(\lambda_{p, q}\right)+d+1$ for some $d \geq 0$. Pick $\mu \in \Lambda_{p, q}^{\circ}$ such that $\mu \stackrel{i}{\rightarrow} \lambda$ is an edge in the crystal graph for some $i \in I_{p, q}$. Then $D^{p, q}(\lambda)$ is the unique irreducible representation of $R_{d+1}^{p, q}$ with the property that $\mathcal{E}_{i} D^{p, q}(\lambda)$ has a quotient isomorphic to $D^{p, q}(\mu)$.

Proof. By Lemma 2.12, we know for $\lambda$ as in the statement and $i \in I_{p, q}$ that $\mathcal{E}_{i} \mathcal{L}(\lambda)$ is zero unless there exists $\mu \in \Lambda_{p, q}^{\circ}$ with $\mu \stackrel{i}{\rightarrow} \lambda$ in the crystal graph, in which case $\mathcal{L}(\mu)$ is the unique irreducible quotient of $\mathcal{E}_{i} \mathcal{L}(\lambda)$. The corollary follows from this on applying the exact functor $\operatorname{Hom}_{G}\left(T_{d}^{p, q}\right.$, ?) and using Lemma 3.25 and the definition (3.19).

Remark 3.27. It is sometimes necessary to understand the homomorphism $\theta_{d ; i}$ from (3.23) from a diagrammatic point of view. Using Theorem 3.12, we can easily write down $\theta_{d ; i}$ on the Khovanov-Lauda-Rouquier generators: it is the map

$$
\theta_{d ; i}: e(\boldsymbol{i}) \mapsto e(\boldsymbol{i}+i), \quad y_{r} e(\boldsymbol{i}) \mapsto y_{r} e(\boldsymbol{i}+i), \quad \psi_{s} e(\boldsymbol{i}) \mapsto \psi_{s} e(\boldsymbol{i}+i)
$$

for $i \in\left(I_{p, q}\right)^{d}, 1 \leq r \leq d$ and $1 \leq s<d$, where $i+i$ denotes the $(d+1)$-tuple $\left(i_{1}, \ldots, i_{d}, i\right)$. It is harder to see $\theta_{d ; i}$ in terms of the bases of oriented stretched circle diagrams, but this is worked out in detail in [BS3, Corollary 6.12]. The basic idea to compute $\theta_{d ; i}\left(\left|\boldsymbol{u}^{*}\left[\boldsymbol{\gamma}^{*}\right] \imath \boldsymbol{t}[\boldsymbol{\delta}]\right|\right)$ is to insert two extra levels chosen from
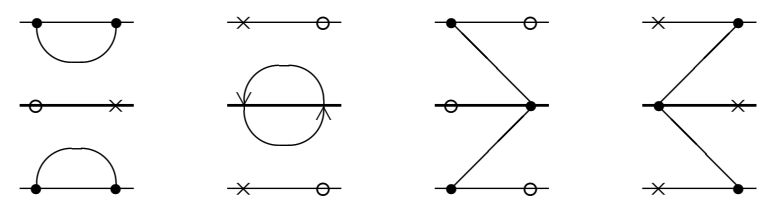

into the middle of the matching $\boldsymbol{u}^{*} \boldsymbol{t}$, where we display only the strip between the $i$ th and $(i+1)$ th vertices, the diagrams being trivial outside that strip. In the first configuration here, this process involves making one application of the generalised surgery procedure. The construction is made precise in the paragraph after [BS3, (6.34)]. 


\section{Morita equivalence with generalised Khovanov algebras}

Next we construct an explicit Morita equivalence between $R^{p, q}:=\bigoplus_{d \geq 0} R_{d}^{p, q}$ and a certain generalised Khovanov algebra $K^{p, q}$. Using this, we replace the tensor space $T^{p, q}:=\bigoplus_{d \geq 0} T_{d}^{p, q}$ from the level two Schur-Weyl duality with a new space $P^{p, q}$ whose endomorphism algebra is $K^{p, q}$. Exploiting the fact that $K^{p, q}$ is a basic algebra, we show that the space $P^{p, q}$ has exactly the same indecomposable summands as $T^{p, q}$ (up to isomorphism), but that they each appear with multiplicity one.

\section{Generalised Khovanov algebras}

Given $p \leq q$, let $K^{p, q}$ denote the subring $e^{p, q} K e^{p, q}$ of $K$, where $e^{p, q}$ is the (non-central) idempotent

$$
e^{p, q}:=\sum_{\lambda \in \Lambda_{p, q}^{\circ}} e_{\lambda} \in K
$$

Lemma 4.1. The algebra $K$ is the union of the subalgebras $K^{p, q}$ for all $p \leq q$.

Proof. This follows from (2.1) and the observation that, for any $\lambda, \mu \in \Lambda$, we can find integers $p \leq q$ such that both $\lambda$ and $\mu$ belong to $\Lambda_{p, q}^{\circ}$.

Remark 4.2. In terms of the diagram basis from (1.7), $K^{p, q}$ has basis

$$
\left\{\begin{array}{l|l}
(a \lambda b) & \begin{array}{l}
\text { for all oriented circle diagrams } a \lambda b \text { with } \lambda \in \Lambda_{p, q} \text { such that } \\
\text { cups and caps pass only through vertices in the interval } I_{p, q}^{+}
\end{array}
\end{array}\right\} .
$$

All the diagrams in the diagram basis of $K^{p, q}$ consist simply of straight lines oriented $\wedge$ outside of the interval $I_{p, q}^{+}$; these play no role when computing the multiplication. So we can just ignore all of the diagram outside this strip without changing the algebra structure. This shows that the algebra $K^{p, q}$ is a direct sum of the generalised Khovanov algebras from [BS1, §6] associated to the weights obtained from $\Lambda_{p, q}$ by erasing vertices outside the interval $I_{p, q}^{+}$.

\section{Representations of $K^{p, q}$}

To understand the representation theory of the algebra $K^{p, q}$, we exploit the exact functor

$$
e^{p, q}: \operatorname{rep}(K) \rightarrow \operatorname{rep}\left(K^{p, q}\right)
$$

arising by left multiplication by the idempotent $e^{p, q}$; cf. [BS1, (6.13)]. It is easy to see that $e^{p, q} L(\lambda) \neq\{0\}$ if and only if $\lambda \in \Lambda_{p, q}^{\circ}$. Hence, letting $L^{p, q}(\lambda):=e^{p, q} L(\lambda)$ for $\lambda \in \Lambda_{p, q}^{\circ}$, the modules

$$
\left\{L^{p, q}(\lambda) \mid \lambda \in \Lambda_{p, q}^{\circ}\right\}
$$

give a complete set of pairwise inequivalent irreducible $K^{p, q}$-modules.

Recalling also the $(K, K)$-bimodules $\widetilde{F}_{i}$ and $\widetilde{E}_{i}$ from $(2.3)$, we get $\left(K^{p, q}, K^{p, q}\right)$ bimodules

$$
\widetilde{F}_{i}^{p, q}:=e^{p, q} \widetilde{F}_{i} e^{p, q}, \quad \widetilde{E}_{i}^{p, q}:=e^{p, q} \widetilde{E}_{i} e^{p, q}
$$


for any $i \in I_{p, q}$. Let $F_{i}:=\widetilde{F}_{i}^{p, q} \otimes_{K}^{p, q}$ ? and $E_{i}:=\widetilde{E}_{i}^{p, q} \otimes_{K^{p, q}}$ ? be the endofunctors of $\operatorname{rep}\left(K^{p, q}\right)$ defined by tensoring with these bimodules.

Lemma 4.3. For any $i \in I_{p, q}$, there are isomorphisms $F_{i} \circ e^{p, q} \cong e^{p, q} \circ F_{i}$ and $E_{i} \circ$ $e^{p, q} \cong e^{p, q} \circ E_{i}$ of functors from $\operatorname{rep}(K)$ to $\operatorname{rep}\left(K^{p, q}\right)$.

Proof. We just explain the proof for $E_{i}$, since the argument for $F_{i}$ is the same. Suppose first that $P$ is any projective right $K$-module that is isomorphic to a direct sum of summands of $e^{p, q} K$. Then the natural multiplication map

$$
P e^{p, q} \otimes_{e^{p, q}} K e^{p, q} e^{p, q} K \rightarrow P
$$

is an isomorphism of right $K$-modules. This follows because it is obviously true if $P=$ $e^{p, q} K$. In the next paragraph, we show that $P=e^{p, q} \widetilde{E}_{i}$ satisfies the hypothesis that it is isomorphic to a direct sum of summands of $e^{p, q} K$ as a right $K$-module. Hence, we deduce that the multiplication map

$$
\widetilde{E}_{i}^{p, q} \otimes_{K} p, q e^{p, q} K \stackrel{\sim}{\rightarrow} e^{p, q} \widetilde{E}_{i}
$$

is a $\left(K^{p, q}, K\right)$-bimodule isomorphism. The desired isomorphism $E_{i} \circ e^{p, q} \cong e^{p, q} \circ E_{i}$ follows at once, since $E_{i} \circ e^{p, q}$ is the functor defined by tensoring with the bimodule on the left hand side and $e^{p, q} \circ E_{i}$ is the functor defined by tensoring with the bimodule on the right hand side of (4.5).

It remains to show that $e^{p, q} \widetilde{E}_{i}$ is isomorphic to a direct sum of summands of $e^{p, q} K$. Equivalently, twisting with the obvious anti-automorphism $*$ that reflects diagrams in a horizontal axis, we show that $\widetilde{F}_{i} e^{p, q}$ is isomorphic to a direct sum of summands of $K e^{p, q}$. The indecomposable summands of $K e^{p, q}$ are all of the form $P(\mu)$ for $\mu \in \Lambda_{p, q}^{\circ}$, so by the definition (4.1) this follows if we can show for any $\lambda \in \Lambda_{p, q}^{\circ}$ that all indecomposable summands of $\widetilde{F}_{i} e_{\lambda}$ are of the form $P(\mu)$ for $\mu \in \Lambda_{p, q}^{\circ}$. As $\widetilde{F}_{i} e_{\lambda} \cong F_{i} P(\lambda)$, this follows easily from [BS2, Theorem 4.2], using also the assumption that $i \in I_{p, q}$.

Corollary 4.4. Let $\lambda, \mu$ and $i$ be as in the statement of Corollary 3.26. Then $L^{p, q}(\lambda)$ is the unique irreducible representation of $K^{p, q}$ with the property that $E_{i} L^{p, q}(\lambda)$ has a quotient isomorphic to $L^{p, q}(\mu)$.

Proof. This follows from Lemmas 2.4 and 4.3 by the same argument used to prove Corollary 3.26 .

\section{Morita bimodules}

Recall the $G$-module $T_{d}^{p, q}$ from (3.7). In view of Theorem 3.21, we can identify its endomorphism algebra with the algebra $R_{d}^{p, q}$. Actually it is convenient now to work with all $d$ simultaneously, setting

$$
\begin{aligned}
T^{p, q} & :=\bigoplus_{d \geq 0} T_{d}^{p, q}, \\
R^{p, q} & :=\bigoplus_{d \geq 0} R_{d}^{p, q} \equiv \operatorname{End}_{G}\left(T^{p, q}\right)^{\mathrm{op}} .
\end{aligned}
$$

Note by Corollary 3.8 and Lemma 3.10 that $T^{p, q}$ and $R^{p, q}$ are both finite-dimensional. 
We next want to explain how the algebra $R^{p, q}$ is Morita equivalent to the basic algebra $K^{p, q}$, by writing down an explicit pair of bimodules $A^{p, q}$ and $B^{p, q}$ that induce the Morita equivalence. To do this, recall the notions of oriented upper- and lower-stretched circle diagrams from $[\mathrm{BS} 3,(6.17)]$. They are the consistently oriented diagrams obtained by gluing a cup diagram below an oriented stretched cap diagram, or gluing a cap diagram above an oriented stretched cup diagram, respectively. Let $A^{p, q}$ and $B^{p, q}$ be the vector spaces with bases

$$
\left.\begin{array}{c|l}
\left\{\begin{array}{l|l}
(a \boldsymbol{t}[\gamma] \mid & \begin{array}{l}
\text { for all oriented upper-stretched circle diagrams } a \boldsymbol{t}[\gamma] \text { of } \\
\text { height } d \geq 0 \text { such that } \gamma_{0}=\lambda_{p, q} \text { and } \gamma_{d} \in \Lambda_{p, q}
\end{array}
\end{array}\right\}, \\
\left\{\mid \boldsymbol{u}^{*}\left[\boldsymbol{\delta}^{*}\right] b\right) & \begin{array}{l}
\text { for all oriented lower-stretched circle diagrams } \boldsymbol{u}^{*}\left[\boldsymbol{\delta}^{*}\right] b \text { of } \\
\text { height } d \geq 0 \text { such that } \delta_{0}=\lambda_{p, q} \text { and } \delta_{d} \in \Lambda_{p, q}
\end{array}
\end{array}\right\},
$$

respectively. We make $A^{p, q}$ into a $\left(K^{p, q}, R^{p, q}\right)$-bimodule as follows.

- The left action of a basis vector $(a \lambda b) \in K^{p, q}$ on $\left(c t[\gamma] \mid \in A^{p, q}\right.$ is by zero unless $\lambda \sim \gamma_{d}$ (where $\gamma=\gamma_{d} \cdots \gamma_{0}$ ) and $b=c^{*}$. Assuming these conditions hold, the product is computed by drawing $a \lambda b$ underneath $c t[\gamma]$, then iterating the generalised surgery procedure to smooth out the symmetric middle section of the diagram.

- The right action of a basis vector $\left|s^{*}\left[\tau^{*}\right]<\boldsymbol{r}[\boldsymbol{\sigma}]\right| \in R^{p, q}$ on $\left(a \boldsymbol{t}[\gamma] \mid \in A^{p, q}\right.$ is by zero unless $t=s$ and all mirror image pairs of internal circles in $s^{*}\left[\tau^{*}\right]$ and $t[\gamma]$ are oriented so that one is clockwise, the other anti-clockwise. Assuming these conditions hold, the product is computed by drawing $a t[\gamma]$ underneath $s^{*}\left[\tau^{*}\right]<r[\sigma]$, erasing all internal circles and number lines in $t[\gamma]$ and $s^{*}\left[\tau^{*}\right]$, then iterating the generalised surgery procedure in the middle section once again.

Similarly we make $B^{p, q}$ into an $\left(R^{p, q}, K^{p, q}\right)$-bimodule. We refer the reader to [BS3, $\S 6]$ for detailed proofs (in an entirely analogous setting) that these bimodules are well defined.

Theorem 4.5. There are isomorphisms

$$
\mu: A^{p, q} \otimes_{R^{p, q}} B^{p, q} \stackrel{\sim}{\rightarrow} K^{p, q}, \quad v: B^{p, q} \otimes_{K^{p, q}} A^{p, q} \stackrel{\sim}{\rightarrow} R^{p, q}
$$

of $\left(K^{p, q}, K^{p, q}\right)$-bimodules and of $\left(R^{p, q}, R^{p, q}\right)$-bimodules, respectively.

Proof. This is a consequence of [BS3, Theorem 6.2 and Remark 6.7]. These references give a somewhat indirect construction of the desired isomorphisms $\mu$ and $\nu$. The same maps can also be constructed much more directly by mimicking the definitions of multiplication in the algebras $R^{p, q}$ and $K^{p, q}$, respectively.

Corollary 4.6 (Morita equivalence). The bimodule $B^{p, q}$ is a projective generator for $\operatorname{rep}\left(R^{p, q}\right)$. Also there is an algebra isomorphism $K^{p, q} \stackrel{\sim}{\rightarrow} \operatorname{End}_{R^{p, q}}\left(B^{p, q}\right)^{\mathrm{op}}$ induced by the right action of $K^{p, q}$ on $B^{p, q}$. Hence the functors

$$
\operatorname{Hom}_{R, q}\left(B^{p, q}, ?\right): \operatorname{rep}\left(R^{p, q}\right) \rightarrow \operatorname{rep}\left(K^{p, q}\right), \quad B^{p, q} \otimes_{K^{p, q}} ?: \operatorname{rep}\left(K^{p, q}\right) \rightarrow \operatorname{rep}\left(R^{p, q}\right)
$$

are quasi-inverse equivalences of categories.

Proof. This follows immediately from the theorem by the usual arguments of the Morita theory; see e.g. [Ba, (3.5) Theorem]. 


\section{More about $i$-restriction and $i$-induction}

We will view the $i$-restriction and $i$-induction functors $\mathcal{E}_{i}$ and $\mathcal{F}_{i}$ from (3.24)-(3.25) now as endofunctors of $\operatorname{rep}\left(R^{p, q}\right)$. Summing the maps $\theta_{d ; i}$ from (3.23) over all $d \geq 0$, we get a unital algebra homomorphism

$$
\theta_{i}: R^{p, q} \rightarrow 1_{i}^{p, q} R^{p, q} 1_{i}^{p, q} \quad \text { where } \quad 1_{i}^{p, q}:=\sum_{d \geq 0} 1_{d ; i}^{p, q}
$$

(which makes sense as the sum has only finitely many non-zero terms). Then $\mathcal{E}_{i}$ is the functor defined by multiplying by the idempotent $1_{i}^{p, q}$, viewing the result as an $R^{p, q}$ module via $\theta_{i}$. The $i$-induction functor $\mathcal{F}_{i}=R^{p, q} 1_{i}^{p, q} \otimes_{R^{p, q}}$ ? is left adjoint to $\mathcal{E}_{i}$; here, we are viewing $R^{p, q} 1_{i}^{p, q}$ as a right $R^{p, q}$-module via $\theta_{i}$.

Lemma 4.7. There is an isomorphism

$$
s^{\prime}: B^{p, q} \otimes_{K} p, q \circ E_{i} \stackrel{\sim}{\rightarrow} \mathcal{E}_{i} \circ B^{p, q} \otimes_{K} p, q ?
$$

of functors from $\operatorname{rep}\left(K^{p, q}\right)$ to $\operatorname{rep}\left(R^{p, q}\right)$.

Proof. By the definitions of the various functors, it suffices to construct an $\left(R^{p, q}, K^{p, q}\right)$ bimodule isomorphism

$$
B^{p, q} \otimes_{K}, \widetilde{E}_{i}^{p, q} \stackrel{\sim}{\rightarrow} 1_{i}^{p, q} B^{p, q},
$$

where $1_{i}^{p, q} B^{p, q}$ is viewed as a left $R^{p, q}$-module via the homomorphism $\theta_{i}$. There is an obvious multiplication map defined on a tensor product of basis vectors of the form $\left.\mid \boldsymbol{u}^{*}\left[\boldsymbol{\delta}^{*}\right] b\right) \otimes(c \lambda t \mu d)$ so that it is zero unless $c=b^{*}$ and $\lambda \sim \delta_{d}$ (where $\delta=\delta_{d} \cdots \delta_{0}$ ), in which case it is the sum of basis vectors obtained by applying the generalised surgery procedure to the $b c$-part of the diagram obtained by putting $\boldsymbol{u}^{*}\left[\delta^{*}\right] b$ underneath $c \lambda t \mu d$. The fact that this multiplication map is an isomorphism of right $K^{p, q}$-modules is a consequence of [BS2, Theorem 3.5]. It remains to show that it is a left $R^{p, q}$-module homomorphism. If we use the diagrammatic description of the map $\theta_{i}$ from Remark 3.27, this reduces to checking a statement which, on applying the anti-automorphism $*$, is equivalent to the identity (6.38) established in the proof of [BS3, Theorem 6.11].

Corollary 4.8. There is an isomorphism

$$
s: E_{i} \circ \operatorname{Hom}_{R} p, q\left(B^{p, q}, ?\right) \stackrel{\sim}{\rightarrow} \operatorname{Hom}_{R^{p, q}}\left(B^{p, q}, ?\right) \circ \mathcal{E}_{i}
$$

of functors from $\operatorname{rep}\left(R^{p, q}\right)$ to $\operatorname{rep}\left(K^{p, q}\right)$.

Proof. In view of Corollary 4.6, the natural transformations arising from the canonical adjunction between tensor and hom give isomorphisms of functors

$$
\begin{aligned}
& \eta: \operatorname{Id}_{\mathrm{rep}\left(K^{p, q}\right)} \stackrel{\sim}{\rightarrow} \operatorname{Hom}_{R^{p, q}}\left(B^{p, q}, ?\right) \circ B^{p, q} \otimes_{K^{p, q}} ?, \\
& \varepsilon: B^{p, q} \otimes_{K^{p, q}} ? \circ \operatorname{Hom}_{R^{p, q}}\left(B^{p, q}, ?\right) \stackrel{\sim}{\rightarrow} \operatorname{Id}_{\operatorname{rep}\left(R^{p, q}\right)} .
\end{aligned}
$$

Now take the isomorphism from Lemma 4.7, compose on the left and the right with the functor $\operatorname{Hom}_{R^{p, q}}\left(B^{p, q}, ?\right)$, and then use the isomorphisms $\eta$ and $\varepsilon$ to cancel the resulting pairs of quasi-inverse functors. 
Identification of irreducible representations

Now we can identify the labelling of the irreducible $R^{p, q}$-modules from Lemma 3.23 with the labelling of the irreducible $K^{p, q}$-modules from (4.3).

Lemma 4.9. For $\lambda \in \Lambda_{p, q}^{\circ}$, we have $\operatorname{Hom}_{R}, q\left(B^{p, q}, D^{p, q}(\lambda)\right) \cong L^{p, q}(\lambda)$ as $K^{p, q}$ modules.

Proof. We first show that $L:=\operatorname{Hom}_{R^{p, q}}\left(B^{p, q}, D^{p, q}\left(\lambda_{p, q}\right)\right) \cong L^{p, q}\left(\lambda_{p, q}\right)$. It is obvious that $\mathcal{E}_{i} D^{p, q}\left(\lambda_{p, q}\right)=\{0\}$ for all $i \in I_{p, q}$. So by Corollary 4.8 we deduce that $E_{i} L=\{0\}$ for all $i \in I_{p, q}$. Combined with Corollary 4.4, this implies that $L \cong L^{p, q}(\mu)$ for some $\mu \in \Lambda_{p, q}^{\circ}$ with ht $(\mu)=\operatorname{ht}\left(\lambda_{p, q}\right)$, hence $L \cong L^{p, q}\left(\lambda_{p, q}\right)$ as $\lambda_{p, q}$ is the only such weight $\mu$.

Now take $\lambda \in \Lambda_{p, q}^{\circ}$ different from $\lambda_{p, q}$, so that $\operatorname{ht}(\lambda)>\operatorname{ht}\left(\lambda_{p, q}\right)$. We again need to show that $L:=\operatorname{Hom}_{R^{p, q}}\left(B^{p, q}, D^{p, q}(\lambda)\right) \cong L^{p, q}(\lambda)$. Let $\mu$ and $i$ be as in Corollary 3.26 , so $D^{p, q}(\mu)$ is a quotient of $\mathcal{E}_{i} D^{p, q}(\lambda)$. We may assume by induction that $\operatorname{Hom}_{R, q}\left(B^{p, q}, D^{p, q}(\mu)\right) \cong L^{p, q}(\mu)$. Applying Corollary 4.8 again, we deduce that $L^{p, q}(\mu)$ is a quotient of $E_{i} L$. So we conclude that $L \cong L^{p, q}(\lambda)$ by Corollary 4.4.

Multiplicity-free version of level two Schur-Weyl duality

Continue with $p \leq q$. Let

$$
P^{p, q}:=T^{p, q} \otimes_{R^{p, q}} B^{p, q} .
$$

This is a $\left(G, K^{p, q}\right)$-bimodule, i.e. it is both a $G$-module and right $K^{p, q}$-module so that the right action of $K^{p, q}$ is by $G$-module endomorphisms.

Theorem 4.10. The homomorphism $K^{p, q} \stackrel{\sim}{\rightarrow} \operatorname{End}_{G}\left(P^{p, q}\right)^{\mathrm{op}}$ induced by the right action of $K^{p, q}$ on $P^{p, q}$ is an isomorphism. Moreover:

(i) There is an isomorphism

$$
\zeta: \operatorname{Hom}_{G}\left(P^{p, q}, ?\right) \stackrel{\sim}{\rightarrow} \operatorname{Hom}_{R}, q\left(B^{p, q}, ?\right) \circ \operatorname{Hom}_{G}\left(T^{p, q}, ?\right)
$$

of functors from $\mathscr{F}$ to $\operatorname{rep}\left(K^{p, q}\right)$.

(ii) There is an isomorphism

$$
t: E_{i} \circ \operatorname{Hom}_{G}\left(P^{p, q}, ?\right) \stackrel{\sim}{\rightarrow} \operatorname{Hom}_{G}\left(P^{p, q}, ?\right) \circ \mathcal{E}_{i}
$$

of functors from $\mathscr{F}$ to $\operatorname{rep}\left(K^{p, q}\right)$.

(iii) We have $\operatorname{Hom}_{G}\left(P^{p, q}, \mathcal{L}(\lambda)\right) \cong L^{p, q}(\lambda)$ for each $\lambda \in \Lambda_{p, q}^{\circ}$.

(iv) As a G-module, $P^{p, q}$ decomposes as $\bigoplus_{\lambda \in \Lambda_{p, q}^{\circ}} P^{p, q} e_{\lambda}$ with $P^{p, q} e_{\lambda} \cong \mathcal{P}(\lambda)$ for each $\lambda \in \Lambda_{p, q}^{\circ}$. 
Proof. We have natural isomorphisms

$$
\begin{aligned}
\operatorname{End}_{G}\left(P^{p, q}\right)^{\mathrm{op}} & =\operatorname{Hom}_{G}\left(T^{p, q} \otimes_{R^{p, q}} B^{p, q}, T^{p, q} \otimes_{R^{p, q}} B^{p, q}\right) \\
& \cong \operatorname{Hom}_{R^{p, q}}\left(B^{p, q}, \operatorname{Hom}_{G}\left(T^{p, q}, T^{p, q} \otimes_{R^{p, q}} B^{p, q}\right)\right) \\
& \cong \operatorname{Hom}_{R^{p, q}}\left(B^{p, q}, \operatorname{Hom}_{G}\left(T^{p, q}, T^{p, q}\right) \otimes_{R^{p, q}} B^{p, q}\right) \\
& \cong \operatorname{Hom}_{R^{p, q}}\left(B^{p, q}, R^{p, q} \otimes_{R^{p, q}} B^{p, q}\right) \cong \operatorname{End}_{R^{p, q}}\left(B^{p, q}\right)^{\mathrm{op}} \cong K^{p, q},
\end{aligned}
$$

using Corollary 4.6. This proves the first statement in the theorem.

Then for (i), we use the natural isomorphisms

$$
\operatorname{Hom}_{G}\left(P^{p, q}, M\right)=\operatorname{Hom}_{G}\left(T^{p, q} \otimes_{R^{p, q}} B^{p, q}, M\right) \cong \operatorname{Hom}_{R^{p, q}}\left(B^{p, q}, \operatorname{Hom}_{G}\left(T^{p, q}, M\right)\right) \text {. }
$$

For (ii), we combine (i), Corollary 4.8 and Lemma 3.25; the isomorphism $t$ is given explicitly by the natural transformation $\zeta^{-1} \mathbf{1} \circ \mathbf{1} r \circ \mathbf{1} \circ \mathbf{1} \zeta$. For (iii), use Lemma 4.9 and the definition (3.19).

Finally, consider (iv). The fact that $P^{p, q}=\bigoplus_{\lambda \in \Lambda_{p, q}^{\circ}} P^{p, q} e_{\lambda}$ follows as the idempotents $\left\{e_{\lambda} \mid \lambda \in \Lambda_{p, q}^{\circ}\right\}$ sum to the identity in $K^{p, q}$. Since $B^{p, q}$ is projective as a left $R^{p, q}$-module, it is a summand of a direct sum of copies of $R^{p, q}$ as a left module. Hence as a $G$-module, $P^{p, q}$ is a summand of a direct sum of copies of $T^{p, q}$. Applying Corollary 3.7, we deduce that the indecomposable summands of $P^{p, q}$ as a $G$-module are all of the form $\mathcal{P}(\lambda)$ for various $\lambda \in \Lambda_{p, q}^{\circ}$. Moreover, for any $\lambda, \mu \in \Lambda_{p, q}^{\circ}$, we have

$$
\operatorname{dim} \operatorname{Hom}_{G}\left(P^{p, q} e_{\lambda}, \mathcal{L}(\mu)\right)=\operatorname{dim} e_{\lambda} \operatorname{Hom}_{G}\left(P^{p, q}, \mathcal{L}(\mu)\right)=\operatorname{dim} e_{\lambda} L^{p, q}(\mu)=\delta_{\lambda, \mu},
$$

using (iii) and the definition of $L^{p, q}(\mu)$. This completes the proof.

\section{Direct limits}

In this section we complete the proof of Theorem 1.1 by taking a limit as $p \rightarrow-\infty$ and $q \rightarrow \infty$.

\section{Various embeddings}

In this subsection we fix $p^{\prime} \leq p \leq q \leq q^{\prime}$ such that either $p^{\prime}=p-1$ and $q^{\prime}=q$, or $p^{\prime}=p$ and $q^{\prime}=q+1$. By definition, the algebra $K^{p, q}$ is equal to the subring $e^{p, q} K^{p^{\prime}, q^{\prime}} e^{p, q}$ of $K^{p^{\prime}, q^{\prime}}$. So $P^{p^{\prime}, q^{\prime}} e^{p, q}$ is a $\left(G, K^{p, q}\right)$-bimodule. The goal is to construct an isomorphism $\pi_{p, q}^{p^{\prime}, q^{\prime}}: P^{p, q} \stackrel{\sim}{\rightarrow} P^{p^{\prime}, q^{\prime}} e^{p, q}$.

Throughout the subsection, we set

$$
i:= \begin{cases}\left(p^{\prime}, p^{\prime}-1, \ldots, p^{\prime}-m+1\right) & \text { if } p^{\prime}=p-1, \\ \left(q^{\prime}, q^{\prime}+1, \ldots, q^{\prime}+n-1\right) & \text { if } q^{\prime}=q+1 .\end{cases}
$$

We have $i \in\left(I_{p^{\prime}, q^{\prime}}\right)^{c}$ where $c:=m$ if $p^{\prime}=p-1$ and $c:=n$ if $q^{\prime}=q+1$. Introduce the idempotent

$$
\xi_{\boldsymbol{i}}:=\sum_{d \geq 0} \xi_{\boldsymbol{i} ; d} \in R^{p^{\prime}, q^{\prime}} \quad \text { where } \quad \xi_{\boldsymbol{i} ; d}=\sum_{\boldsymbol{j} \in\left(I_{p, q}\right)^{d}} e(\boldsymbol{i}+\boldsymbol{j}) \in R_{c+d}^{p^{\prime}, q^{\prime}},
$$


writing $\boldsymbol{i}+\boldsymbol{j}$ for the sequence $\left(i_{1}, \ldots, i_{c}, j_{1}, \ldots, j_{d}\right)$. The following lemma explains how to identify $R^{p, q}$ with the subring $\xi_{\boldsymbol{i}} R^{p^{\prime}, q^{\prime}} \xi_{\boldsymbol{i}}$ of $R^{p^{\prime}, q^{\prime}}$.

Lemma 5.1. Let $\boldsymbol{t}=t_{c} \cdots t_{1}$ be the composite matching and $\mathbf{o}=\Gamma_{c} \cdots \Gamma_{0}$ be the block sequence associated to the $\left(p^{\prime}, q^{\prime}\right)$-admissible sequence $i$ from (5.1). Let $\gamma=\gamma_{c} \cdots \gamma_{0}$ be the unique sequence of weights with $\gamma_{r} \in \Gamma_{r}$ for each $r$; in particular, $\gamma_{0}=\lambda_{p^{\prime}, q^{\prime}}$ and $\gamma_{c}=\lambda_{p, q}$. Then there is a unital algebra isomorphism

$$
\rho_{p, q}^{p^{\prime}, q^{\prime}}: R^{p, q} \stackrel{\sim}{\rightarrow} \xi_{\boldsymbol{i}} R^{p^{\prime}, q^{\prime}} \xi_{\boldsymbol{i}}
$$

defined on the basis of oriented stretched circle diagrams by setting

$$
\rho_{p, q}^{p^{\prime}, q^{\prime}}\left(\left|\boldsymbol{s}^{*}\left[\boldsymbol{\tau}^{*}\right]<\boldsymbol{r}[\boldsymbol{\sigma}]\right|\right):=\mid \boldsymbol{t}^{*}\left[\gamma^{*}\right]\left\llcorner\boldsymbol{s}^{*}\left[\boldsymbol{\tau}^{*}\right]<\boldsymbol{r}[\boldsymbol{\sigma}]<\boldsymbol{t}[\gamma] \mid,\right.
$$

i.e. we glue $\gamma_{0} t_{1}^{*} \gamma_{1} \cdots \gamma_{c-1} t_{c}^{*}$ onto the bottom and $t_{c} \gamma_{c-1} \cdots \gamma_{1} t_{1} \gamma_{0}$ onto the top of the given diagram $\boldsymbol{s}^{*}\left[\boldsymbol{\tau}^{*}\right] \geq \boldsymbol{r}[\boldsymbol{\sigma}]$. Moreover, if we write $\rho_{p, q}^{p^{\prime}, q^{\prime}}=\sum_{d \geq 0} \rho_{d}$ for isomorphisms $\rho_{d}: R_{d}^{p, q} \stackrel{\sim}{\rightarrow} \xi_{i ; d} R_{c+d}^{p^{\prime}, q^{\prime}} \xi_{i ; d}$, the following two properties hold.

(i) On the Khovanov-Lauda-Rouquier generators of $R_{d}^{p, q}$, we have

$$
\rho_{d}(e(\boldsymbol{j}))=e(\boldsymbol{i}+\boldsymbol{j}), \quad \rho_{d}\left(\psi_{r}\right)=\xi_{\boldsymbol{i} ; d} \psi_{c+r}, \quad \rho_{d}\left(y_{s}\right)=\xi_{\boldsymbol{i} ; d} y_{c+s},
$$

for $j \in\left(I_{p, q}\right)^{d}, 1 \leq r<d$ and $1 \leq s \leq d$.

(ii) On the Hecke generators of $R_{d}^{p, q}$, we have

$$
\rho_{d}\left(s_{r}\right)=\xi_{i ; d} s_{c+r}, \quad \rho_{d}\left(x_{s}\right)=\xi_{i ; d} x_{c+s},
$$

for $1 \leq r<d$ and $1 \leq s \leq d$.

Proof. The existence of the isomorphism $\rho_{p, q}^{p^{\prime}, q^{\prime}}$ is a consequence of the diagrammatic description of the algebras $R^{p, q}$ and $\xi_{\boldsymbol{i}} R^{p^{\prime}, q^{\prime}} \xi_{i}$. One first checks by inspecting bases that the given linear map is a vector space isomorphism, then that it preserves multiplication. The latter is obvious because we have just added some extra line segments all oriented $\wedge$ at the top and bottom of the diagram.

To check (i), it follows from (3.12) and the definitions just before (3.15) and (3.14) that $\rho_{d}(e(\boldsymbol{j}))=e(\boldsymbol{i}+\boldsymbol{j}), \rho_{d}\left(\bar{\psi}_{r}\right)=\xi_{\boldsymbol{i} ; d} \bar{\psi}_{c+r}$ and $\rho_{d}\left(\bar{y}_{s}\right)=\xi_{\boldsymbol{i} ; d} \bar{y}_{c+s}$. It remains to show that the signs (3.13) involved in passing from $\bar{\psi}$ to $\psi$ and from $\bar{y}$ to $y$ match up correctly, which amounts to the observation that $\sigma_{p, q}^{r}(\boldsymbol{j})=\sigma_{p^{\prime}, q^{\prime}}^{c+r}(\boldsymbol{i}+\boldsymbol{j})$ for $\boldsymbol{j} \in\left(I_{p, q}\right)^{d}$ and $1 \leq r \leq d$. This follows from the identity

$$
\min \left(p, j_{r}\right)+\min \left(q, j_{r}\right)-p=\min \left(p^{\prime}, j_{r}\right)+\min \left(q^{\prime}, j_{r}\right)-p^{\prime}-\delta_{i_{1}, j_{r}}-\cdots-\delta_{i_{c}, j_{r}},
$$

which we leave as an exercise. Then (ii) follows from (i) and Theorem 3.12.

We can make a very similar construction at the level of the bimodule $B^{p, q}$. In the following lemma, we view $\xi_{i} B^{p^{\prime}, q^{\prime}} e^{p, q}$ as an $\left(R^{p, q}, K^{p, q}\right)$-bimodule, where the left $R^{p, q}$ module structure is defined via the isomorphism from Lemma 5.1. 
Lemma 5.2. Let $\boldsymbol{t}$ and $\gamma$ be as in Lemma 5.1. There is an isomorphism of $\left(R^{p, q}, K^{p, q}\right)$ bimodules

$$
\beta_{p, q}^{p^{\prime}, q^{\prime}}: B^{p, q} \stackrel{\sim}{\rightarrow} \xi_{i} B^{p^{\prime}, q^{\prime}} e^{p, q}
$$

defined on the basis of oriented upper-stretched circle diagrams by setting

$$
\left.\left.\beta_{p, q}^{p^{\prime}, q^{\prime}}\left(\mid \boldsymbol{u}^{*}\left[\boldsymbol{\delta}^{*}\right] b\right)\right):=\mid \boldsymbol{t}^{*}\left[\boldsymbol{\gamma}^{*}\right] \prec \boldsymbol{u}^{*}\left[\boldsymbol{\delta}^{*}\right] b\right) .
$$

Moreover, the map

$$
\kappa_{p, q}^{p^{\prime}, q^{\prime}}: R^{p^{\prime}, q^{\prime}} \xi_{i} \otimes_{R^{p, q}} B^{p, q} \rightarrow B^{p^{\prime}, q^{\prime}} e^{p, q}, \quad x \otimes b \mapsto x \beta_{p, q^{p^{\prime}, q^{\prime}}(b),}
$$

is an isomorphism of $\left(R^{p^{\prime}, q^{\prime}}, K^{p, q}\right)$-bimodules.

Proof. The fact that $\beta_{p, q}^{p^{\prime}, q^{\prime}}$ is an isomorphism of vector spaces follows by considering the explicit diagram bases, and it is obviously a bimodule homomorphism. To deduce the final part of the lemma, it remains to show that the natural multiplication map

$$
R^{p^{\prime}, q^{\prime}} \xi_{\boldsymbol{i}} \otimes_{\xi_{i} R^{p^{\prime}, q^{\prime}} \xi_{i}} \xi_{\boldsymbol{i}} B^{p^{\prime}, q^{\prime}} e^{p, q} \rightarrow B^{p^{\prime}, q^{\prime}} e^{p, q}
$$

is an isomorphism. For this, we argue as in the proof of Lemma 4.3, starting from the trivial observation that the multiplication map

$$
R^{p^{\prime}, q^{\prime}} \xi_{\boldsymbol{i}} \otimes_{\xi_{\boldsymbol{i}} R^{p^{\prime}, q^{\prime}} \xi_{\boldsymbol{i}}} \xi_{\boldsymbol{i}} R^{p^{\prime}, q^{\prime}} \xi_{\boldsymbol{i}} \rightarrow R^{p^{\prime}, q^{\prime}} \xi_{\boldsymbol{i}}
$$

is an isomorphism. Thus, we are reduced to showing that all the indecomposable summands of $B^{p^{\prime}, q^{\prime}} e^{p, q}$ are also summands of $R^{p^{\prime}, q^{\prime}} \xi_{i}$ as left $R^{p^{\prime}, q^{\prime}}$-modules. By Corollary 4.6 and Lemma 4.9 , we know that the indecomposable summands of $B^{p^{\prime}, q^{\prime}} e^{p, q}$ are the projective covers of the irreducible $R^{p^{\prime}, q^{\prime}}$-modules $\left\{D^{p^{\prime}, q^{\prime}}(\lambda) \mid \lambda \in \Lambda_{p, q}^{\circ}\right\}$. Since $R^{p^{\prime}, q^{\prime}} \xi_{\boldsymbol{i}}$ is projective, it just remains to check that

$$
\operatorname{Hom}_{R^{p^{\prime}}, q^{\prime}}\left(R^{p^{\prime}, q^{\prime}} \xi_{\boldsymbol{i}}, D^{p^{\prime}, q^{\prime}}(\lambda)\right)=\xi_{\boldsymbol{i}} D^{p^{\prime}, q^{\prime}}(\lambda) \neq\{0\}
$$

for $\lambda \in \Lambda_{p, q}^{\circ}$. By Lemma 2.6, we can find $d \geq 0$ and a tuple $j \in\left(I_{p, q}\right)^{d}$ such that $\lambda_{p, q} \stackrel{j_{1}}{\rightarrow}$ $\ldots \stackrel{j_{d}}{\rightarrow} \lambda$ is a path in the crystal graph. As $\lambda_{p^{\prime}, q^{\prime}} \stackrel{i_{1}}{\rightarrow} \ldots \stackrel{i_{c}}{\rightarrow} \lambda_{p, q}$ is a path in the crystal graph too, repeated application of Corollary 3.26 shows that $\mathcal{E}_{i_{1}} \cdots \mathcal{E}_{i_{c}} \mathcal{E}_{j_{1}} \cdots \mathcal{E}_{j_{d}} D^{p^{\prime}, q^{\prime}}(\lambda)$ $\neq\{0\}$. By the definition of the $i$-restriction functors, this means $e(i+j) D^{p^{\prime}, q^{\prime}}(\lambda) \neq\{0\}$. Since $\xi_{i} e(i+j)=e(i+j)$, this implies that $\xi_{i} D^{p^{\prime}, q^{\prime}}(\lambda) \neq\{0\}$ too.

Next we explain how to identify $T^{p, q}$ with $T^{p^{\prime}, q^{\prime}} \xi_{\boldsymbol{i}}$.

Lemma 5.3. There exists a (unique up to scalars) $G$-module isomorphism

$$
\tau_{p, q}^{p^{\prime}, q^{\prime}}: T^{p, q} \stackrel{\sim}{\rightarrow} T^{p^{\prime}, q^{\prime}} \xi_{i}
$$

such that $\tau_{p, q^{p^{\prime}}}^{p^{\prime}}=\sum_{d \geq 0} \tau_{d}$ for isomorphisms $\tau_{d}: T_{d}^{p, q} \stackrel{\sim}{\rightarrow} T_{c+d}^{p^{\prime}, q^{\prime}} \xi_{i ; d}$ with $\tau_{d+1}=$ $\sum_{k \in I_{p, q}} \mathcal{F}_{k}\left(\tau_{d}\right)$ for each $d \geq 0$. Moreover, $\tau_{p, q^{p^{\prime}}}^{p^{\prime}}$ is a homomorphism of right $R^{p, q_{-}}$ modules, i.e. it is a $\left(G, R^{p, q}\right)$-bimodule isomorphism, where we are viewing $T^{p^{\prime}, q^{\prime}} \xi_{i}$ as a right $R^{p, q}$-module via the isomorphism from Lemma 5.1. 
Proof. We first construct the map $\tau_{0}$. Recall that $T_{0}^{p, q}=\mathcal{V}\left(\lambda_{p, q}\right)$ and $T_{c}^{p^{\prime}, q^{\prime}} \xi_{i ; 0}=$ $\left(\mathcal{V}\left(\lambda_{p^{\prime}, q^{\prime}}\right) \otimes V^{\otimes c}\right) e(\boldsymbol{i})=\mathcal{F}_{\boldsymbol{i}} \mathcal{V}\left(\lambda_{p^{\prime}, q^{\prime}}\right)$. By Lemma 2.12 we have $\mathcal{F} \boldsymbol{i} \mathcal{V}\left(\lambda_{p^{\prime}, q^{\prime}}\right) \cong \mathcal{V}\left(\lambda_{p, q}\right)$; only the analogues of the statements from (i) and (iii) of Lemma 2.4 are needed to see this. So we can pick a $G$-module isomorphism

$$
\tau_{0}: T_{0}^{p, q}=\mathcal{V}\left(\lambda_{p, q}\right) \stackrel{\sim}{\rightarrow} \mathcal{F}_{i} \mathcal{V}\left(\lambda_{p^{\prime}, q^{\prime}}\right)=T_{c}^{p^{\prime}, q^{\prime}} \xi_{\boldsymbol{i} ; 0} .
$$

This map is unique up to a scalar.

Now we inductively define the higher $\tau_{d}$ 's. Since $T_{d}^{p, q}=\bigoplus_{\boldsymbol{j} \in\left(I_{p, q}\right)^{d}} \mathcal{F}_{\boldsymbol{j}} \mathcal{V}\left(\lambda_{p, q}\right)$ we have $T_{d+1}^{p, q}=\bigoplus_{k \in I_{p, q}} \mathcal{F}_{k} T_{d}^{p, q}$. Similarly $T_{c+d+1}^{p^{\prime}, q^{\prime}} \xi_{i ; d+1}=\bigoplus_{k \in I_{p, q}} \mathcal{F}_{k}\left(T_{c+d}^{p^{\prime}, q^{\prime}} \xi_{i ; d}\right)$. So given a $G$-module isomorphism $\tau_{d}: T_{d}^{p, q} \stackrel{\sim}{\rightarrow} T_{c+d}^{p^{\prime}, q^{\prime}} \xi_{\boldsymbol{i} ; d}$ for some $d \geq 0$, we get a $G$ module isomorphism $\tau_{d+1}: T_{d+1}^{p, q} \stackrel{\sim}{\rightarrow} T_{c+d+1}^{p^{\prime}, q^{\prime}} \xi_{\boldsymbol{i} ; d+1}$ on applying the functor $\bigoplus_{k \in I_{p, q}} \mathcal{F}_{k}$. Starting from the map $\tau_{0}$ from the previous paragraph, we obtain isomorphisms $\tau_{d}$ for every $d \geq 0$ in this way. Then we set $\tau_{p, q}^{p^{\prime}, q^{\prime}}:=\sum_{d \geq 0} \tau_{d}$, to get the desired $G$-module isomorphism $T^{p, q} \stackrel{\sim}{\rightarrow} T^{p^{\prime}, q^{\prime}} \xi_{\boldsymbol{i}}$.

It remains to check that each $\tau_{d}$ is a homomorphism of right $R_{d}^{p, q}$-modules, viewing $T_{c+d}^{p^{\prime}, q^{\prime}} \xi_{\boldsymbol{i} ; d}$ as a right $R_{d}^{p, q}$-module via the isomorphism $\rho_{d}$ from Lemma 5.1. Because $\tau_{0}$ is a $G$-module homomorphism, the map

$\mathcal{V}\left(\lambda_{p, q}\right) \otimes V^{\otimes d} \rightarrow \mathcal{V}\left(\lambda_{p^{\prime}, q^{\prime}}\right) \otimes V^{\otimes(c+d)}, \quad u \otimes v \mapsto \tau_{0}(u) \otimes v\left(u \in \mathcal{V}\left(\lambda_{p, q}\right), v \in V^{\otimes d}\right)$, intertwines the action of $x_{s} \in H_{d}$ with $x_{c+s} \in H_{c+d}$. It obviously intertwines the action of each $s_{r} \in H_{d}$ with $s_{c+r} \in H_{c+d}$. From this and the definition of $\tau_{d}$, we deduce that $\tau_{d}$ intertwines the actions of $s_{r}, x_{s} \in H_{d}$ on $T_{d}^{p, q}$ with the actions of $s_{c+r}, x_{c+s} \in H_{c+d}$ on $T_{c+d}^{p^{\prime}, q^{\prime}} \xi_{\boldsymbol{i} ; d}$. So we are done by the description of $\rho_{d}$ from Lemma 5.1(ii).

Recall finally the spaces $P^{p, q}=T^{p, q} \otimes_{R^{p, q}} B^{p, q}$ from (4.9).

Theorem 5.4. There is a unique (up to scalars) $\left(G, K^{p, q}\right)$-bimodule isomorphism

$$
\pi_{p, q}^{p^{\prime}, q^{\prime}}: P^{p, q} \stackrel{\sim}{\rightarrow} P^{p^{\prime}, q^{\prime}} e^{p, q}
$$

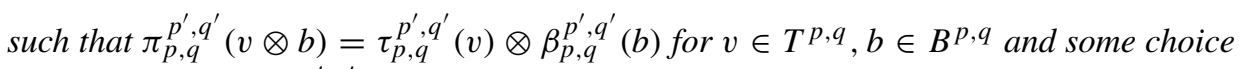
of the isomorphism $\tau_{p, q}^{p^{\prime}, q^{\prime}}$ from Lemma 5.3.

Proof. Recalling the isomorphism $\kappa_{p, q}^{p^{\prime}, q^{\prime}}$ from Lemma 5.2, we define $\pi_{p, q}^{p^{\prime}, q^{\prime}}$ to be the composition of the following $\left(G, K^{p, q}\right)$-bimodule isomorphisms:

$$
\begin{aligned}
T^{p, q} \otimes_{R}^{p, q} B^{p, q} \stackrel{\tau_{p, q}^{p^{\prime}, q^{\prime}} \otimes \mathrm{id}}{\longrightarrow} T^{p^{\prime}, q^{\prime}} \xi_{\boldsymbol{i}} \otimes_{R, q} B^{p, q} \equiv T^{p^{\prime}, q^{\prime}} \otimes_{R_{p^{\prime}, q^{\prime}}} R^{p^{\prime}, q^{\prime}} \xi_{\boldsymbol{i}} \otimes_{R}, q B^{p, q} \\
\stackrel{\operatorname{id} \otimes \kappa_{p, q}^{p^{\prime}, q^{\prime}}}{\longrightarrow} T^{p^{\prime}, q^{\prime}} \otimes_{R^{p^{\prime}, q^{\prime}}} B^{p^{\prime}, q^{\prime}} e^{p, q} .
\end{aligned}
$$

It remains to observe that $\pi_{p, q}^{p^{\prime}, q^{\prime}}(v \otimes b)=\tau_{p, q^{p^{\prime}}, q^{\prime}}(v) \otimes \beta_{p, q^{\prime}}^{p^{\prime}, q^{\prime}}(b)$, which follows from the definition of $\kappa_{p, q}^{p^{\prime}, q^{\prime}}$. 


\section{Compatibility of embeddings}

Now we explain how to glue the isomorphisms $\pi_{p, q}^{p, q+1}$ and $\pi_{p, q}^{p-1, q}$ from the previous subsection together in a consistent way to obtain a compatible system of isomorphisms $\pi_{p, q}^{p^{\prime}, q^{\prime}}: P^{p, q} \stackrel{\sim}{\rightarrow} P^{p^{\prime}, q^{\prime}} e^{p, q}$ for every $p^{\prime} \leq p \leq q \leq q^{\prime}$. The following lemma is the key ingredient making this possible.

Lemma 5.5. Let $p \leq q$ be fixed. Given a choice of three out of the four maps

$$
\left\{\pi_{p, q}^{p, q+1}, \pi_{p, q}^{p-1, q}, \pi_{p, q+1}^{p-1, q+1}, \pi_{p-1, q}^{p-1, q+1}\right\}
$$

from Theorem 5.4, there is a unique way to choose the fourth one so that $\pi_{p, q+1}^{p-1, q+1}$ 。 $\pi_{p, q}^{p, q+1}=\pi_{p-1, q}^{p-1, q+1} \circ \pi_{p, q}^{p-1, q}$.

Proof. We show equivalently given a choice of all four maps that there is a (necessarily unique) scalar $z \in \mathbb{F}$ such that

$$
\pi_{p, q+1}^{p-1, q+1} \circ \pi_{p, q}^{p, q+1}=z \pi_{p-1, q}^{p-1, q+1} \circ \pi_{p, q}^{p-1, q} .
$$

To see this, let $\boldsymbol{h}:=(p-1, p-2, \ldots, p-m)$ and $i:=(q+1, q+2, \ldots, q+n)$. Let

$$
\begin{aligned}
\psi & :=\left(\psi_{m} \psi_{m+1} \cdots \psi_{m+n-1}\right) \cdots\left(\psi_{2} \psi_{3} \cdots \psi_{n+1}\right)\left(\psi_{1} \psi_{2} \cdots \psi_{n}\right), \\
\psi^{\prime} & :=\left(\psi_{n} \cdots \psi_{2} \psi_{1}\right)\left(\psi_{n+1} \cdots \psi_{3} \psi_{2}\right) \cdots\left(\psi_{m+n-1} \cdots \psi_{m+1} \psi_{m}\right) .
\end{aligned}
$$

It is easy to see from the defining relations between the Khovanov-Lauda-Rouquier generators from [BS3, (6.8)-(6.16)] that $\psi \xi_{\boldsymbol{i}+\boldsymbol{h}}=\xi_{\boldsymbol{h}+\boldsymbol{i}} \psi, \xi_{\boldsymbol{i}+\boldsymbol{h}} \psi^{\prime}=\psi^{\prime} \xi_{\boldsymbol{h}+\boldsymbol{i}}$, and $\psi^{\prime} \psi \xi_{\boldsymbol{i}+\boldsymbol{h}}=\xi_{\boldsymbol{i}+\boldsymbol{h}}$ in $R^{p-1, q+1}$.

Now we claim that there exists a scalar $z \in \mathbb{F}$ such that the following two diagrams commute:

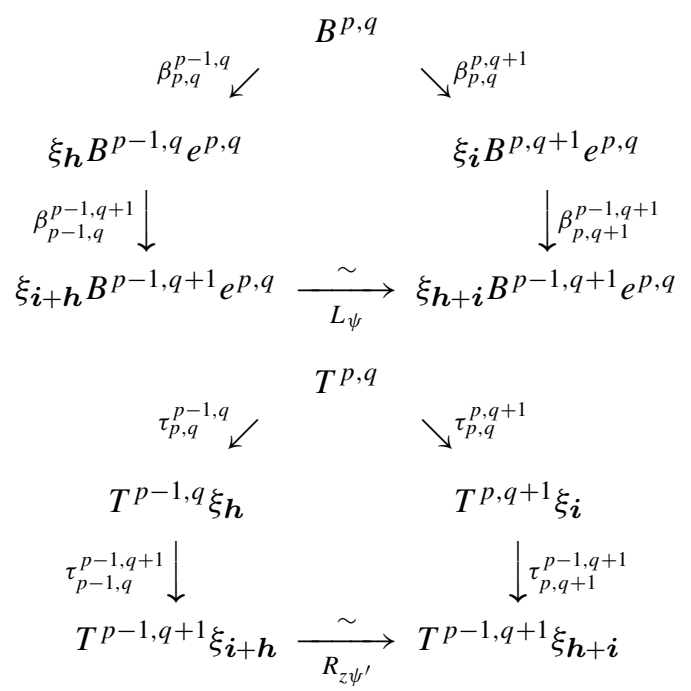


where $L_{\psi}(b):=\psi b$ and $R_{z \psi^{\prime}}(v):=z v \psi^{\prime}$. Given the claim and recalling Lemma 5.4, we see for any $v \otimes b \in T^{p, q} \otimes_{R^{p, q}} B^{p, q}$ that

$$
\begin{aligned}
\pi_{p, q+1}^{p-1, q+1}\left(\pi_{p, q}^{p, q+1}(v \otimes b)\right) & =\tau_{p, q+1}^{p-1, q+1}\left(\tau_{p, q}^{p, q+1}(v)\right) \otimes \beta_{p, q+1}^{p-1, q+1}\left(\beta_{p, q}^{p, q+1}(b)\right) \\
& =z \tau_{p-1, q}^{p-1, q+1}\left(\tau_{p, q}^{p-1, q}(v)\right) \psi^{\prime} \otimes \psi \beta_{p-1, q}^{p-1, q+1}\left(\beta_{p, q}^{p-1, q}(b)\right) \\
& =z \tau_{p-1, q}^{p-1, q+1}\left(\tau_{p, q}^{p-1, q}(v)\right) \otimes \psi^{\prime} \psi \beta_{p-1, q}^{p-1, q+1}\left(\beta_{p, q}^{p-1, q}(b)\right) \\
& =z \tau_{p-1, q}^{p-1, q+1}\left(\tau_{p, q}^{p-1, q}(v)\right) \otimes \beta_{p-1, q}^{p-1, q+1}\left(\beta_{p, q}^{p-1, q}(b)\right) \\
& =z \pi_{p-1, q}^{p-1, q+1}\left(\pi_{p, q}^{p-1, q}(v \otimes b)\right) .
\end{aligned}
$$

So the lemma follows from the claim.

To prove the claim, consider first the diagram (5.3). The point for this is that all the $\psi_{r}$ 's in the element $\psi$ are acting successively on the left on $\xi_{\boldsymbol{i}+\boldsymbol{h}} B^{p-1, q+1} e^{p, q}$ as a sequence of height moves in the sense of [BS3, §5]. Combined with the diagrammatic definition from Lemma 5.3 this is enough to see that (5.3) commutes. Next consider the diagram (5.4). Here one first reduces using the definition of the higher $\tau_{d}$ 's in Lemma 5.3 to checking just that the diagram commutes on restriction to $T_{0}^{p, q}=\mathcal{V}\left(\lambda_{p, q}\right)$. In that case, both of $T_{m+n}^{p-1, q+1} \xi_{\boldsymbol{i}+\boldsymbol{h}}$ and $T_{m+n}^{p-1, q+1} \xi_{\boldsymbol{h}+\boldsymbol{i}}$ are isomorphic to $\mathcal{V}\left(\lambda_{p, q}\right)$, and the map defined by right multiplication by $\psi^{\prime}$ is a non-zero isomorphism. So the diagram must commute up to a scalar as $\operatorname{End}_{G}\left(\mathcal{V}\left(\lambda_{p, q}\right)\right)$ is one-dimensional.

Theorem 5.6. We can choose $\left(G, K^{p, q}\right)$-bimodule isomorphisms

$$
\pi_{p, q}^{p^{\prime}, q^{\prime}}: P^{p, q} \stackrel{\sim}{\rightarrow} P^{p^{\prime}, q^{\prime}} e^{p, q}
$$

for all $p^{\prime} \leq p \leq q \leq q^{\prime}$ in such a way that $\pi_{p, q^{p^{\prime \prime}}, q^{\prime \prime}}=\pi_{p^{\prime}, q^{\prime}}^{p^{\prime \prime}, q^{\prime \prime}} \circ \pi_{p, q}^{p^{\prime}, q^{\prime}}$ whenever $p^{\prime \prime} \leq$ $p^{\prime} \leq p \leq q \leq q^{\prime} \leq q^{\prime \prime}$

Proof. First of all we make arbitrary choices for the maps $\pi_{p, q}^{p, q+1}$ from Theorem 5.4 for all $p \leq q$. Also we make arbitrary choices for the maps $\pi_{p, p}^{p-1, p}$ from Theorem 5.4 for all $p$. Then we repeatedly apply Lemma 5.5, proceeding by induction on $q-p$, to get maps $\pi_{p, q}^{p-1, q}$ such that the following local relation holds:

$$
\pi_{p, q+1}^{p-1, q+1} \circ \pi_{p, q}^{p, q+1}=\pi_{p-1, q}^{p-1, q+1} \circ \pi_{p, q}^{p-1, q}
$$

for all $p \leq q$. Finally we define the maps $\pi_{p, q^{\prime}}^{p^{\prime}, q^{\prime}}$ in general by setting $\pi_{p, q}^{p^{\prime}, q^{\prime}}:=\pi_{p^{\prime}+1, q^{\prime}}^{p^{\prime}, q^{\prime}}$ 。

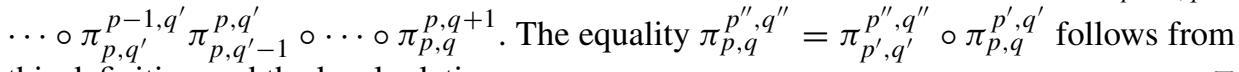
this definition and the local relation.

\section{Proof of the main theorem}

Consider the directed set $\{(p, q) \mid p \leq q\}$ where $(p, q) \rightarrow\left(p^{\prime}, q^{\prime}\right)$ if $p^{\prime} \leq p \leq q \leq q^{\prime}$. By Theorem 5.6, it is possible to choose a direct system $\left\{\pi_{p, q^{p^{\prime}}, q^{\prime}}: P^{p, q} \rightarrow P^{p^{\prime}, q^{\prime}} e^{p, q}\right\}$ of 
$\left(G, K^{p, q}\right)$-bimodule isomorphisms for every $(p, q) \rightarrow\left(p^{\prime}, q^{\prime}\right)$. Let

$$
P:=\lim _{\longrightarrow} P^{p, q}
$$

be the corresponding direct limit taken in the category of all $G$-modules, and denote the canonical inclusion of each $P^{p, q}$ into $P$ by $\varphi^{p, q}$. We make $P$ into a locally unital right $K$-module as follows. Take $x \in K$ and $v \in P$. Recalling Lemma 4.1, we can choose $p \leq q$ so that $x=e^{p, q} x e^{p, q}$ and $v=\varphi^{p, q}\left(v^{p, q}\right)$ for some $v^{p, q} \in P^{p, q}$. Then set $v x:=\varphi^{p, q}\left(v^{p, q} x\right)$.

Remark 5.7. Note that $P$ is independent of the particular choice of the maps $\left\{\pi_{p, q}^{p^{\prime}, q^{\prime}}\right\}$ in the sense that if $\bar{P}=\lim _{\longrightarrow} P^{p, q}$ is another such direct limit taken with respect to maps $\left\{\bar{\pi}_{p, q}^{p^{\prime}, q^{\prime}}\right\}$, then there is a unique bimodule isomorphism $P \stackrel{\sim}{\rightarrow} \bar{P}$ such that $\varphi^{p, q}(v) \mapsto$ $\bar{\varphi}^{p, q}(v)$ for all $v \in P^{p, q}$ and $p \leq q$.

Roughly speaking, the following lemma shows that $P$ is a minimal projective generator for the category $\mathscr{F}$ (except that as $P$ is not finite-dimensional it is not actually an object in the category).

Lemma 5.8. As a $G$-module, we have $P=\bigoplus_{\lambda \in \Lambda} P e_{\lambda}$ with $P e_{\lambda} \cong \mathcal{P}(\lambda)$ for each $\lambda \in \Lambda$.

Proof. The first part of the lemma is immediate because $P$ is a locally unital right $K$ module. To show that $P e_{\lambda} \cong \mathcal{P}(\lambda)$, we find by the above definitions that $P e_{\lambda}=$ $\underset{\lim _{\lambda}}{\longrightarrow}\left(P^{p, q} e_{\lambda}\right)$ where the direct limit is taken over all $p \leq q$ with $\lambda \in \Lambda_{p, q}^{\circ}$ (so that $\overrightarrow{e_{\lambda}} \in K^{p, q}$ ). Each $P^{p, q} e_{\lambda}$ is isomorphic to $\mathcal{P}(\lambda)$ by Theorem 4.10(iv). Hence the direct limit is isomorphic to $\mathcal{P}(\lambda)$ too.

Now we want to identify the algebra $K$ with the endomorphism algebra of $P$. A little care is needed here as $P$ is an infinite direct sum. So for any $G$-module $M$, we let

$$
\operatorname{Hom}_{G}^{\mathrm{fin}}(P, M):=\bigoplus_{\lambda \in \Lambda} \operatorname{Hom}_{G}\left(P e_{\lambda}, M\right) \subseteq \operatorname{Hom}_{G}(P, M),
$$

which is the locally finite part of $\operatorname{Hom}_{G}(P, M)$. If $M$ is finite-dimensional then $\operatorname{Hom}_{G}^{\mathrm{fin}}(P, M)=\operatorname{Hom}_{G}(P, M)$. In particular, we denote $\operatorname{Hom}_{G}^{\mathrm{fin}}(P, P)$ by $\operatorname{End}_{G}^{\mathrm{fin}}(P)$ and write $\operatorname{End}_{G}^{\mathrm{fin}}(P)^{\mathrm{op}}$ for the opposite algebra, which acts naturally on the right on $P$ by $G$-module endomorphisms.

Lemma 5.9. The right action of $K$ on $P$ defined above induces an algebra isomorphism $K \stackrel{\sim}{\rightarrow} \operatorname{End}_{G}^{\mathrm{fin}}(P)^{\mathrm{op}}$.

Proof. We need to show that right multiplication induces a vector space isomorphism $e_{\lambda} K \stackrel{\sim}{\rightarrow} \operatorname{Hom}_{G}\left(P e_{\lambda}, P\right)$ for each $\lambda \in \Lambda$. By definition, the right hand space is

$$
\operatorname{Hom}_{G}\left(P e_{\lambda}, \bigcup P e^{p, q}\right)=\bigcup \operatorname{Hom}_{G}\left(P e_{\lambda}, P e^{p, q}\right)
$$


where we can take the union just over $p \leq q$ with $\lambda \in \Lambda_{p, q}^{\circ}$. As $P e_{\lambda}=\varphi^{p, q}\left(P^{p, q} e_{\lambda}\right)$ and $P e^{p, q}=\varphi^{p, q}\left(P^{p, q}\right)$ for all such $p \leq q$, the first statement from Theorem $4.10 \mathrm{im}$ plies that right multiplication induces an isomorphism $e_{\lambda} K e^{p, q} \stackrel{\sim}{\rightarrow} \operatorname{Hom}_{G}\left(P e_{\lambda}, P e^{p, q}\right)$. Taking the union and recalling Lemma 4.1, we deduce that we do get an isomorphism $e_{\lambda} K \stackrel{\sim}{\rightarrow} \cup \operatorname{Hom}_{G}\left(P e_{\lambda}, P e^{p, q}\right)$.

Finally we record the following variation on a basic fact.

Lemma 5.10. Let $B$ be a $G$-module that is also a locally unital right $K$-module, such that the action of $K$ on $B$ is by $G$-module endomorphisms. Let $M$ be any finite-dimensional left $K$-module and assume that $B \otimes_{K} M$ is finite-dimensional. Then there is a natural $G$-module isomorphism

$$
\operatorname{Hom}_{G}^{\text {fin }}(P, B) \otimes_{K} M \rightarrow \operatorname{Hom}_{G}\left(P, B \otimes_{K} M\right)
$$

sending $f \otimes m$ to the homomorphism $v \mapsto f(v) \otimes m$.

Proof. It suffices to show that $\operatorname{Hom}_{G}\left(P e_{\lambda}, B\right) \otimes_{K} M \cong \operatorname{Hom}_{G}\left(P e_{\lambda}, B \otimes_{K} M\right)$ for each $\lambda \in \Lambda$, which is well known.

Now we can prove the main result of the article, which is essentially Theorem 1.1 from the introduction with the functor $\mathbb{E}$ there constructed explicitly. The proof is a rather standard consequence of the last three lemmas, but we include some details since we are in a slightly unusual locally finite setting.

Theorem 5.11. The functors

$$
\operatorname{Hom}_{G}(P, ?): \mathscr{F} \rightarrow \operatorname{rep}(K), \quad P \otimes_{K} ?: \operatorname{rep}(K) \rightarrow \mathscr{F}
$$

are quasi-inverse equivalences of categories. Moreover $P \otimes_{K} P(\lambda) \cong \mathcal{P}(\lambda)$ for each $\lambda \in \Lambda$.

Proof. By Lemma 5.8 both the functors map finite-dimensional modules to finite-dimensional modules, so the first statement makes sense. Lemmas 5.10 and 5.9 yield a natural isomorphism

$$
\operatorname{Hom}_{G}\left(P, P \otimes_{K} M\right) \stackrel{\sim}{\rightarrow} \operatorname{Hom}_{G}^{\text {fin }}(P, P) \otimes_{K} M \cong K \otimes_{K} M \equiv M
$$

for any $M \in \operatorname{rep}(K)$. Thus $\operatorname{Hom}_{G}(P, ?) \circ P \otimes_{K}$ ? $\cong \operatorname{Id}_{\text {rep }(K)}$. Conversely, to show that $P \otimes_{K}$ ? $\circ \operatorname{Hom}_{G}(P, ?) \cong \operatorname{Id}_{\mathscr{F}}$, we have a natural homomorphism

$$
P \otimes_{K} \operatorname{Hom}_{G}(P, N) \rightarrow N, \quad v \otimes f \mapsto f(v),
$$

for every $N \in \mathscr{F}$. Because of Lemma 5.8 this map is surjective. To show that it is injective too, denote its kernel by $U$. Applying the exact functor $\operatorname{Hom}_{G}(P, ?)$, we get a short exact sequence

$$
0 \rightarrow \operatorname{Hom}_{G}(P, U) \rightarrow \operatorname{Hom}_{G}\left(P, P \otimes_{K} \operatorname{Hom}_{G}(P, N)\right) \rightarrow \operatorname{Hom}_{G}(P, N) \rightarrow 0 .
$$

By the fact established just before, the middle space here is isomorphic to $\operatorname{Hom}_{G}(P, N)$, so the right hand map is an isomorphism. Hence $\operatorname{Hom}_{G}(P, U)=\{0\}$, which implies that 
$U=\{0\}$. So our natural transformation is an isomorphism, and we have established the equivalence of categories. Moreover,

$$
P \otimes_{K} P(\lambda)=P \otimes_{K} K e_{\lambda} \equiv P e_{\lambda} \cong \mathcal{P}(\lambda)
$$

by Lemma 5.8 .

Theorem 1.1 from the introduction is a consequence of Theorem 5.11 for $\mathbb{E}:=$ $\operatorname{Hom}_{G}(P$, ?). We have already proved that $\mathbb{E} \mathcal{P}(\lambda) \cong P(\lambda)$, which immediately implies that $\mathbb{E} \mathcal{L}(\lambda) \cong L(\lambda)$. The fact that $\mathbb{E} \mathcal{V}(\lambda) \cong V(\lambda)$ follows because both the categories $\mathscr{F}$ and $\operatorname{rep}(K)$ are highest weight categories in which the modules $\{\mathcal{V}(\lambda)\}$ and $\{V(\lambda)\}$ give the standard modules; see Theorem 2.15 for the former and [BS1, Theorem 5.3] for the latter fact.

\section{Identification of special projective functors}

Finally we discuss briefly how to relate the special projective functors on the two sides of our equivalence of categories.

Theorem 5.12. For each $i \in I$, we have

$$
E_{i} \cong \operatorname{Hom}_{G}(P, ?) \circ \mathcal{E}_{i} \circ P \otimes_{K} ?, \quad F_{i} \cong \operatorname{Hom}_{G}(P, ?) \circ \mathcal{F}_{i} \circ P \otimes_{K} ?
$$

as endofunctors of $\operatorname{rep}(K)$.

Proof. Since $F_{i}$ is left adjoint to $E_{i}$ and $\mathcal{F}_{i}$ is left adjoint to $\mathcal{E}_{i}$, the second isomorphism is a consequence of the first, by unicity of adjoints. To prove the first, we note using Lemma 5.10 that there are natural isomorphisms

$$
\operatorname{Hom}_{G}\left(P, \mathcal{E}_{i}\left(P \otimes_{K} M\right)\right) \cong \operatorname{Hom}_{G}\left(P,\left(\mathcal{E}_{i} P\right) \otimes_{K} M\right) \cong \operatorname{Hom}_{G}^{\mathrm{fin}}\left(P, \mathcal{E}_{i} P\right) \otimes_{K} M
$$

for any $M \in \operatorname{rep}(K)$. Hence it suffices to show that $\operatorname{Hom}_{G}{ }_{\text {fin }}\left(P, \mathcal{E}_{i} P\right) \cong \widetilde{E}_{i}$ as $(K, K)$ bimodules. For this, we just sketch how to construct the appropriate map, leaving details to the reader. Take any $\lambda \in \Lambda$ and any $p \leq q$ so that we actually have $\lambda \in \Lambda_{p, q}^{\circ}$. When applied to the module $P^{p, q}$, the natural isomorphism from Theorem 4.10(ii) produces a $\left(K^{p, q}, K^{p, q}\right)$-bimodule isomorphism

$$
\varepsilon^{p, q}: \widetilde{E}_{i}^{p, q} \stackrel{\sim}{\rightarrow} \operatorname{Hom}_{G}\left(P^{p, q}, \mathcal{E}_{i} P^{p, q}\right) .
$$

Restricting this to $e_{\lambda} \widetilde{E}_{i}^{p, q}=e_{\lambda} \widetilde{E}_{i} e^{p, q}$ and using $\varphi^{p, q}$ to identify $P^{p, q}$ with $P e^{p, q}$, we get from this a vector space isomorphism

$$
\varepsilon^{p, q}: e_{\lambda} \widetilde{E}_{i} e^{p, q} \stackrel{\sim}{\rightarrow} e_{\lambda} \operatorname{Hom}_{G}\left(P e^{p, q}, \mathcal{E}_{i} P e^{p, q}\right)=\operatorname{Hom}_{G}\left(P e_{\lambda}, \mathcal{E}_{i} P e^{p, q}\right) .
$$

Now one checks for $p^{\prime} \leq p \leq q \leq q^{\prime}$ that $\varepsilon^{p, q}(v)=\varepsilon^{p^{\prime}, q^{\prime}}(v)$ for all $v \in e_{\lambda} \widetilde{E}_{i} e^{p, q}$; it suffices to do this in the cases $\left(p^{\prime}, q^{\prime}\right)=(p-1, q)$ or $(p, q+1)$. Hence it makes sense to take the union over all $p \leq q$ to get an isomorphism

$$
\varepsilon: e_{\lambda} \widetilde{E}_{i} \stackrel{\sim}{\rightarrow} \operatorname{Hom}_{G}\left(P e_{\lambda}, \mathcal{E}_{i} P\right)
$$


Taking the direct sum of these maps over all $\lambda \in \Lambda$ gives finally the desired map $\widetilde{E}_{i} \stackrel{\sim}{\rightarrow}$ $\operatorname{Hom}_{G}^{\text {fin }}\left(P, \mathcal{E}_{i} P\right)$.

Acknowledgments. This article was written up during stays by both authors at the Isaac Newton Institute in Spring 2009. We thank the INI staff and the Algebraic Lie Theory programme organisers for the opportunity.

The first author was supported in part by NSF grant no. DMS-0654147.

\section{Index of notation}

$G=G L(m \mid n) \quad$ General linear supergroup $\quad 373$

$V, V^{*} \quad$ Natural representation of $G$ and its dual $\quad 374$

$\begin{array}{lll}B, T & \text { Standard Borel subgroup and maximal torus of } G & 374\end{array}$

$\mathscr{F}=\mathscr{F}(m \mid n) \quad$ Half of the category of finite-dimensional $G$-modules $\quad 374$

$\begin{array}{lll}X^{+}(T) & \text { Dominant weights } & 374\end{array}$

$\mathcal{L}(\lambda), \mathcal{V}(\lambda), \mathcal{P}(\lambda) \quad$ Irreducibles, standards and PIMs for $G$ for $\lambda \in X^{+}(T)$

$\mathcal{E}_{i}, \mathcal{F}_{i} \quad$ Special projective functors for $G \quad 385$

$K=K(m \mid n) \quad$ Generalised Khovanov algebra $\quad 380$

$\Lambda=\Lambda(m \mid n) \quad$ Diagrammatic weights in bijection with $X^{+}(T) \quad 374$

$L(\lambda), V(\lambda), P(\lambda) \quad$ Irreducibles, standards and PIMs for $K$ for $\lambda \in \Lambda$

$E_{i}, F_{i} \quad$ Special projective functors for $K$

$\begin{array}{lll}\lambda_{p, q} & \text { Ground-state weight } & 376\end{array}$

$H_{d}^{p, q} \quad$ Cyclotomic Hecke algebra which acts on $\mathcal{V}\left(\lambda_{p, q}\right) \otimes V^{\otimes d} \quad 391$

$I_{p, q}^{+} \quad$ Index set for $p q$-strip $\quad 383$

$\Lambda_{p, q}, \Lambda_{p, q}^{\circ} \quad$ Weights, weights of maximal defect in $p q$-strip 383

$1_{d}^{p, q} \quad$ Central idempotent in $H_{d}^{p, q}$ corresponding to $p q$-strip 392

$T_{d}^{p, q} \quad$ Tensor space $\left(\mathcal{V}\left(\lambda_{p, q}\right) \otimes V^{\otimes d}\right) 1_{d}^{p, q}$

$R_{d}^{p, q} \quad$ Cyclotomic KLR algebra $\cong 1_{d}^{p, q} H_{d}^{p, q} \cong \operatorname{End}_{G}\left(T_{d}^{p, q}\right)^{\mathrm{op}}$

$T^{p, q}, R^{p, q} \quad$ Direct sums $\bigoplus_{d \geq 0} T_{d}^{p, q}$ and $\bigoplus_{d \geq 0} R_{d}^{p, q} \quad 405$

$K^{p, q} \quad$ Subring of $K$ that is Morita equivalent to $R^{p, q} \quad 404$

$A^{p, q}, B^{p, q} \quad$ Morita bimodules 406

$P^{p, q} \quad$ Multiplicity-free projective module $T^{p, q} \otimes_{R^{p, q}} B^{p, q} \quad 408$

$P=\lim _{\longrightarrow} P^{p, q} \quad$ Canonical minimal projective generator for $\mathscr{F} \quad 415$

\section{References}

[Ba] Bass, H.: Algebraic $K$-Theory. Benjamin, New York (1968) Zbl 0174.30302 MR 0249491

[BGS] Beilinson, A., Ginzburg, V., Soergel, W.: Koszul duality patterns in representation theory, J. Amer. Math. Soc. 9, 473-527 (1996) Zbl 0864.17006 MR 1322847

[BKN] Boe, B., Kujawa, J., Nakano, D.: Complexity and module varieties for classical Lie superalgebras. Int. Math. Res. Notices 2011, no. 3, 696-724 Zbl pre05859695 MR 2764876

[B1] Brundan, J.: Kazhdan-Lusztig polynomials and character formulae for the Lie superalgebra $\mathfrak{g l}(m \mid n)$. J. Amer. Math. Soc. 16, 185-231 (2003) Zbl 1050.17004 MR 1937204

[B2] Brundan, J.: Tilting modules for Lie superalgebras. Comm. Algebra 32, 2251-2268 (2004) Zbl 1077.17006 MR 2100468 
[BK1] Brundan, J., Kleshchev, A.: Schur-Weyl duality for higher levels. Selecta Math. 14, 1-57 (2008) Zbl 1211.17012 MR 2480709

[BK2] Brundan, J., Kleshchev, A.: Blocks of cyclotomic Hecke algebras and Khovanov-Lauda algebras. Invent. Math. 178, 451-484 (2009) Zbl 1201.20004 MR 2551762

[BS1] Brundan, J., Stroppel, C.: Highest weight categories arising from Khovanov's diagram algebra I: cellularity. Moscow Math. J. 11, 685-722 (2011)

[BS2] Brundan, J., Stroppel, C.: Highest weight categories arising from Khovanov's diagram algebra II: Koszulity. Transform. Groups 15, 1-45 (2010) Zbl 1205.17010 MR 2600694

[BS3] Brundan, J., Stroppel, C.: Highest weight categories arising from Khovanov's diagram algebra III: Category $\mathcal{O}$. Represent. Theory 15, 170-243 (2011) MR 2781018

[CKL] Cheng, S.-J., Kwon, J.-H., Lam, N.: A BGG-type resolution for tensor modules over general linear superalgebra. Lett. Math. Phys. 84, 75-87 (2008) Zbl 1172.17005 MR 2393055

[CL] Cheng, S.-J., Lam, N.: Irreducible characters of general linear superalgebra and super duality. Comm. Math. Phys. 298, 645-672 (2010) Zbl 1217.17004 MR 2670923

[CW] Cheng, S.-J., Wang, W.: Brundan-Kazhdan-Lusztig and super duality conjectures. Publ. Res. Inst. Math. Sci. 44, 1219-1272 (2008) Zbl 1210.17009 MR 2477902

[CWZ] Cheng, S.-J., Wang, W., Zhang, R. B.: Super duality and Kazhdan-Lusztig polynomials. Trans. Amer. Math. Soc. 360, 5883-5924 (2008) Zbl pre05358271 MR 2425696

[CPS] Cline, E., Parshall, B., Scott, L.: Finite dimensional algebras and highest weight categories. J. Reine Angew. Math. 391, 85-99 (1988) Zbl 0657.18005 MR 0961165

[D] Drinfeld, V.: Degenerate affine Hecke algebras and Yangians. Funct. Anal. Appl. 20, 69-70 (1986) MR 0831053

[Dr] Drouot, F.: Quelques propriétés des représentations de la super-algèbre de Lie $\mathfrak{g l}(m, n)$. PhD thesis, Université Henri Poincaré, Nancy (2008)

[Ka] Kac, V.: Characters of typical representations of classical Lie superalgebras. Comm. Algebra 5, 889-897 (1977) Zbl 0359.17010 MR 0444725

[Kh] Khovanov, M.: A functor-valued invariant of tangles. Algebr. Geom. Topol. 2, 665-741 (2002) Zbl 1002.57006 MR 1928174

[KL] Khovanov, M., Lauda, A.: A diagrammatic approach to categorification of quantum groups I. Represent. Theory 13, 309-347 (2009) Zbl 1188.81117 MR 2525917

[Ro] Rouquier, R.: 2-Kac-Moody algebras. arXiv:0812.5023

[Se1] Serganova, V.: Kazhdan-Lusztig polynomials and character formula for the Lie superalgebra $\mathfrak{g l}(m \mid n)$. Selecta Math. 2, 607-651 (1996) Zbl 0881.17005 MR 1443186

[Se2] Serganova, V.: Characters of irreducible representations of simple Lie superalgebras. In: Proc. Int. Congress of Mathematicians (Berlin, 1998), Vol. II, 583-593 Zbl 0898.17002 MR 1648107

[St1] Stroppel, C.: Category $\mathcal{O}$ : quivers and endomorphism rings of projectives. Represent. Theory 7, 322-345 (2003) Zbl 1050.17005 MR 2017061

[St2] Stroppel, C.: Parabolic category $\mathcal{O}$, perverse sheaves on Grassmannians, Springer fibres and Khovanov homology. Compos. Math. 145, 954-992 (2009) Zbl 1187.17004 MR 2521250

[St3] Stroppel, C.: Schur-Weyl duality and link homology. In: Proc. Int. Congress of Mathematicians (Hyderabad, 2010), Vol. III, 1344-1365

[Zo] Zou, Y. M.: Categories of finite dimensional weight modules over type I classical Lie superalgebras. J. Algebra 180, 459-482 (1996) Z Zbl 0843.17001 MR 1378540 